\title{
Through the Looking Glass:
}

An investigation of physical materials in virtual space

Kimberly Moh

A 120 point thesis submitted to the Victoria University of Wellington in partial fulfilment of the requirements for the degree of Master of Architecture (Professional)

Victoria University of Wellington

School of Architecture 
Special thanks to my supervisor Tane Moleta for your many insightful and interesting discussions, your exuberant encouragements and guidance throughout the entire year.

To my amazing parents who have been so supportive and understanding throughout my entire education at Victoria University.

To my lovely friends and the boys from my thesis stream, thank you for keeping me sane and providing many laughs amidst our stress. 


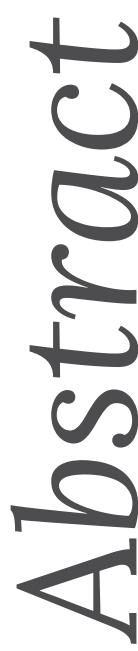

This thesis questions, what characteristics constitute architecture and how can a design based body of research push the boundaries to question standard convention of architecture? My research proposes that there is a lost opportunity in conventional architecture design when the limitation of two dimensional images can not fully convey the reality of space. Conventionally architecture is viewed as static and unchanging however, this research disagree with this proposition and explores the opportunity for there to be an immersive experience throughout the design stage. This research project asks how can we bridge the gap between the architecture created in the physical world versus the virtual?

Scaled physical modeling is traditionally the key visual representation of architectural design. However, people often can't fully understand the intricacies of a scaled model. Through my research, I explore how VR technology could provide solutions to let people experience the materiality and craft of a real life model. Restrictions in perception of scale, materiality and circulation hinders the thorough understanding of experience in space.

There is an opportunity to collaborate and merge the experience of physical modeling in the digital realm. By using hand crafted physical models, my work brings the imperfection of real life into the digital realm. Allowing the materiality from the real world to ground the project with the familiarity that only real space can induce.

Paper craft and modeling by card are the chosen material and method of constructing these real life scaled models. Photogrammetry is used as a process to translate these physical "objects" into digital mesh. The physical and virtual world can work together harmoniously to create interesting results. The material properties of physical models are highlighted but have the advantage of being able to be easily manipulated (scale, multiply, move etc) in the digital realm. By using VR in order to explore these created environments allows the users to grasp the full nature of the materials and craftsmanship of physical models in a 1:1 scale. This hybrid way of designing celebrates the benefits and advantage of both the physical and virtual world and is a start to bridge the gap between them. 
Acknowledgement

Abstract

Chapter 1: Introduction

Chapter 2: Literature Review

Representation

Physical vs Digital Model

Chapter 3: Case Studies

Lumino City

Tengami

Laika Films

Theatre Sets

Chapter 4: Preliminary Design

Exploration 1

Exploration 2

Exploration 3

Unity

Scale

Chapter 5: Design Process

Process

Physical Model

Photogrammetry Content

Scene 1

Scene 2

Scene 3

Scene 4

Scene 5

Chapter 6: Conclusion

Bibliography

List of Figures

Appendix 
"Begin at the beginning," the King said, very gravely, "and go on till you come to the end: then stop."

- Lewis Carroll, Alice Adventure in Wonderland, 1865 


\section{Chapter 1: \\ Introduction}


Model making has been the staple of architecture education since the twentieth century (Morris, 2006) and is a conventional tool that architects and students alike can understand easily. However as the world of architecture transitions into the digital age, the art of hand-made models has been lost. Physical materials and craftsmanship has an unique characteristic that cannot be easily replicated in the digital format. There is an imperfection to the human touch that grounds people to reality (Cheng 1995). Digital modeling has the potential to evoke a sense of being too perfect and too manufactured. The term 'Uncanny Valley' was coined as a descriptor of when the digital medium produces 'near' photographic replicas of real life environments or products that hinge on the edge of the unrealistic, in turn making people uncomfortable (Schwarz, 2013). There are benefits to both analog and digital ways of designing. This research questions, why one would need to choose one or the other when they could be used in conjunction with each other. Such a combination would bring about the advantages of both methods and collaborating between the two different mediums could demonstrate the positive outcome they provide.

I have always found a fascination with learning new software applications and how they might benefit or provide an alternative way of designing and thinking throughout my education. Virtual Reality (VR) as a format for design was the direction I have taken for my thesis topic. VR technology has advanced to the point where the digital medium can mimic real life characteristics more precisely. VR places an occupant in a digital environment that has the ability to adjust according to set parameters. This form of technology can counteract the restrictions of traditional physical modeling. The concept of VR has been around for a few decades but only recently been a factor in the architecture industry. Now, content creation VR programs have been developed for artist and creators alike. Why can it not be used to understand architecture? The 1:1 scale exploration of preexisting and current architecture and environments has been a big draw for consumers. It is an extension of a digital and physical scale model built to use as a way to solve problem and represent the architecture before it is built. Why not take advantage of the abilities to see more during the concept phase of the design process.

Another factor that narrowed the direction in which I chose my thesis topic was my interest in video games and the cinema. I had always found myself fascinated with the way architecture has been framed and utilised outside the immediate discipline. The world building and the freedom of creating architectural spaces and environments based on narrative ideas is an appealing concept. I wanted to take all these aspects I find compelling and turn them into a thesis topic I will enjoy undertaking for the year. 
How can Real-Time Virtual Environments be used to bridge the gap between material properties of the physical world and the perceived lack of materiality of the digital?

This question inquiries on the advantages and disadvantages of both physical and digital modes of design in architecture. It focus on the materiality of the physical world and examine how that might enhance the experience of the virtual space. Using RTVE as a way to test this allows the observers to take agency in how they view and experience the overall design instead of relying on static images to represent the final outcome. The objective of this is to critically examine the benefits of using VR in the design phase of architecture allowing for time, memory and motion to enhance our experience of space. 


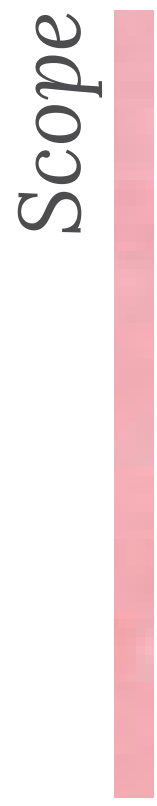

This thesis is an exploration of materiality properties and craftsmanship in virtual space. It is about the attempt to bridge the gap between the material properties of the physical and virtual world and explore a new hybrid nature that exists as an amalgam of both realities. The experimental qualities of Virtual Reality (VR) space using physically made content, testing the benefits of scaled models compared to the digital based geometry are thoroughly researched. This thesis is not an attempt to design a final "building" but instead various environments are created as a testing ground to study the usefulness and effectiveness of physical material properties. The design was heavily process based using scaled modeling, photogrammetry and VR (in relation to understanding VR editing tools, and VR compositing) in order to test the research question. VR is used not just as a visualisation tool but by using the unique features, it provides an immersive experience to enhance the properties of physical materiality.

The thesis is based on a marking criteria of $75 \%$ design and $25 \%$ written text.

Academic texts are typically written in the impartial first person or completely avoiding the use of personal pronoun in order to conduct an objective tone. However, as this thesis is focused on research through design and the topic was formed through my own interest of the subject, personal pronoun has been used throughout this dissertation. This decision was made as a product of finding my position in the theoretical and designresearch based context of the chosen thesis topic which helped to shape the decisions and judgments made throughout the entire design process. 
The main process in the new thesis structure is a process based research (or in other words research through design). This emphasis on the particular method is an advantage for the creative discipline as we, as designers are constantly learning through the creation process. Design inform knowledge and throughout that knowledge, new direction and judgements can be made to proceed further. Research based on textual and knowledge gather from outside mediums are also important in offering a reference into directing the path of the chosen topic.

An established narrative of Alice in Wonderland has been chosen as a testing ground for this thesis. In choosing an existing story, it allows the focus to remain on the research topic of physical materiality properties in digital space. Physical hand crafted models is the approach used for this experiment and paper is the chosen material in which the physical models will be constructed with. Paper is a versatile modeling material and keeping with a single material will ensure the design remain consistent and coherent throughout. Papercraft and origami are the intended aesthetic of choice.

Different methods are needed to translate and convert physical models (and their material properties) into the digital realm. Photogrammetry is the main method used while programs such as AutoDesk Remake and AutoDesk 3dsMax are used to clean and modify the final result. The process is repeated with various models made in order to create a full 'library' of elements in order to construct a virtual environment.

All these components are assembled using Unity (a real-time virtual engine) in order to produce virtual environment that can be inhabited. Texture, lighting, sound and digital ephemera (Moleta 2017) are incorporated into the digital realm in order to enhance the overall ambience of the space. These elements bring a depth to architecture environment that is often lost in still images. Virtual Reality is used as an exploration tool for users and observers to inhabit the created environments in a 1:1 scale. 
This thesis is structured around a research through design framework with a general theoretical context to guide the direction in which the thesis will be understood.

Chapter 1 - Introduction: This chapter outlines the background, scope and methodology and serves as an introduction for the thesis topic.

Chapter 2 - Literature Review: Theoretical context is provided in determining the direction and justification of the thesis topic. It is used to establish a conceptual framework for the research. It covers topic such as representation and the limitation of stilled images, physical scaled model in the digital age, and the advantages of mixing the two realities.

Chapter 3 - Case Studies: Four precedents across different mediums are analysed and assessed based on relevancy to the thesis topic. These case studies from games to film to theatre are important in showcasing materiality and agency of person outside of conventional architecture standards. Helpful information are drawn from these exemplars in influencing the thesis research.

Chapter 4 - Preliminary Design: This chapter documents the initial design exploration in relation to the research question. New knowledge are gained through the design investigation leading to constant assessment and refinement of the direction of the research.

Chapter 5 - Design Process: This chapter extends on the knowledge learnt from the previous chapter. It showcase the process from the making of the physical models to translating and implementing them in a virtual environment. This final design (through the use of Virtual Reality) is a critical response to the research question and have materialised together from various information gathered throughout the thesis process.

Chapter 6 - Discussion and Reflection: This chapter provides a focussed discussion and reflection of the entire process of the thesis document. It reflects on the effectivity of the investigation through the design process, the strength and weaknesses, and places in which improvement can be made. Discussions are made on the relevancy of the thesis topic and how it can fit within the role of architecture in today's context. 


\section{Chapter 2:}

\section{Literature Review}


This chapter provides a theoretical background on representation and the discourse of physical vs digital. It will be used to build an argument for the use of VR and real-world material based modeling. The limitation of the static image is explored and reviewed as architecture space is more than what is portrayed through the two dimensional representation methods prevalent in architecture education and practice today. It expands to find alternative methods of representation that encapsulate representation in the third dimension using the technologies available to us. Advantages and disadvantages of physical and digital models are also analysed with regards to their process of creation and the materiality properties of both mediums. Each section ends with a conclusive understanding of what I will be taking from these theoretical readings into my design research. 
Different modes of representation have been present throughout architecture history, but one of the most prominent forms of representation in recent age is that of the representation through visual material. Two dimensional images are used extensively to communicate visual information; either through the design process to convey ideas and concepts, or through a photo realistic rendered (fig.01), or through architecture photography (fig.02) showcasing the building itself. However, there is a trend of the image being presented to us of architecture that is a misrepresentation of the building in reality. Computer generated images (CGI) and architectural photographs are people's first exposure to architecture instead of the built form in reality. Luis Alfonso de la Fuente Suarez (2016) argued that the image being represented isn't the reality of the architecture but the experience of the objects.

Though I agree with the intent I believe the limitation of static images hinders how we, as architects can convey the true scope of the architecture designed. The still image cannot be comparable to the real architectural object and unfortunately the majority of what architecture has become in the last few decades is just a series of images representing the built form. The final outcome presented reduces the architectural object of representation into a linear abstraction as light and shadow, reflection and transparency, binocular and peripheral vision are compressed together in a single image (Allen, 2009).

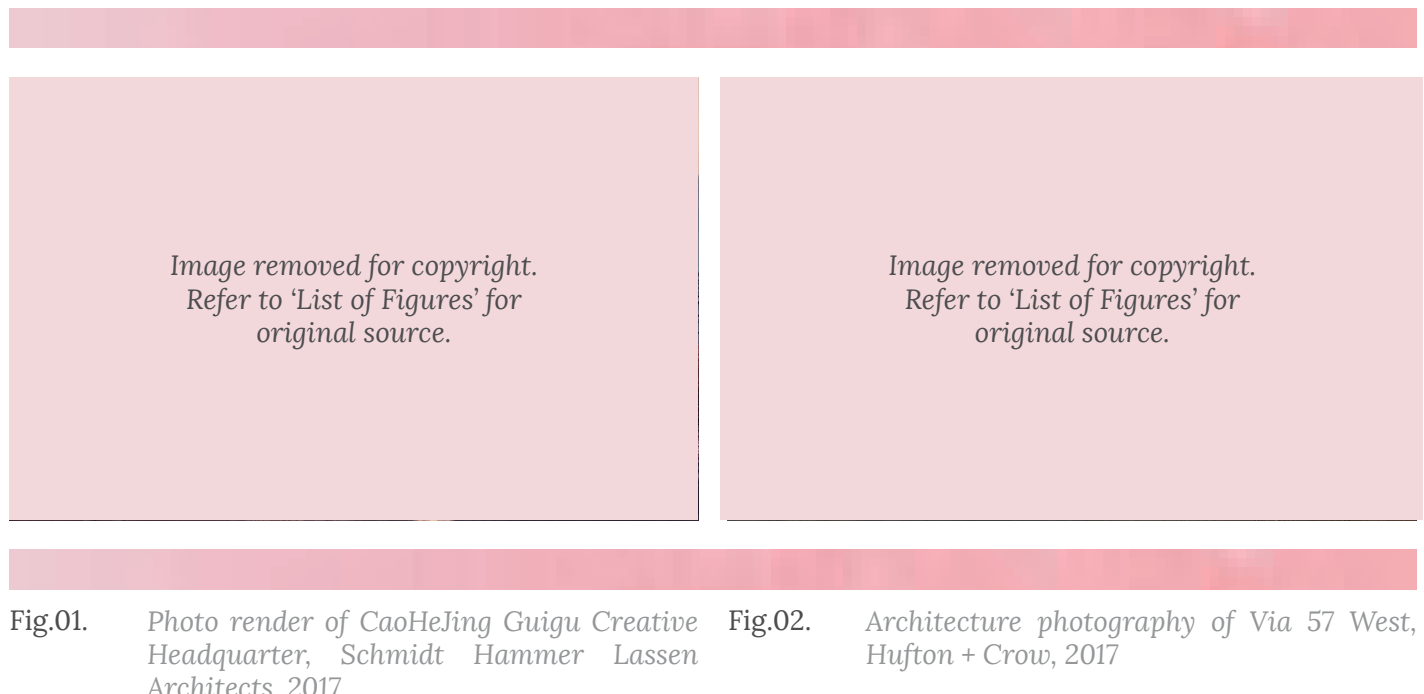


Many architecture theorists such as Juhani Pallasmaa have stated that experiencing a design in the third dimension is where we can fully appreciate the space. He has criticised the printed image and the way in which "... the gaze itself tends to flatten into a picture and lose its plasticity' instead of experiencing our being in the world, we behold it from outside as spectators of images projected on the surface of the retina." (Holl, Pallasmaa and Perez-Gomez, 2006, p. 29). The two-dimensional image is limited and only through the third dimension can the full intent of the architectural design be realised.

Through the post-digital age, many images or representation we see can be deceiving to the eye. Jonathan Openshaw (2015) states this in his book Post Digital Artisans,

"The act of seeing has always been fallible to an extent, but with digital manipulation there are fresh opportunities for perception to be distorted, disrupted and manipulated. The act of seeing requires some considerable deciphering of complex and conflicting messages, becoming an action or interaction rather than a passive reflection." (p. 8)

Due to the fact that images and photographs could be manipulated, we are being forced to understand an 'altered' version of the architecture. This leaves understanding based upon the representation up to the interpretation of the observer and can thus build up expectations that are not delivered in the final outcome (Knuuttila, 2005). Thus, by introducing alternative methods of representation beyond the static image, it can help diminish the gap of interpretation by the designer and observer. 
In this, the discussion of representation theory has a number of key theoretical precedents. We cannot just rely on the eye of static images to convey all forms of information to us as designers want to communicate. These arguments have pushed me to reconsider how representation is typically used throughout my architecture education and how there are alternative methods to the static image. Using forms of animation and cinematic techniques can bring motion into the representation to showcase more what is intended in the architecture that has been designed. Although they break from the static entity of most 2D images, these forms of representation also have their limitations. "... a specific route has been pre-selected that focuses on specific experiential aspects and objects of the environment" (Fuente Suarez, 2016, p. 52). They do not allow their observers to have agency over the environment but instead are refined to only show certain aspects of the project which in turn limits the full understanding of an architectural space (fig.03).

The immersive quality is a missing element in majority of represented work, by introducing Virtual Reality into my methods of designing, this will elevate the outcome to be more than just the 'image'. Immersive virtual reality allows the observer to experience the designed environment at a near human perceptual scale, exploring the intended atmosphere of the pre-existing environment in a three dimensional scope. This form of representation is the closest observers can get to understand the effective space, therefore showcasing more accurately the intentions behind the design than a two dimensional image. 


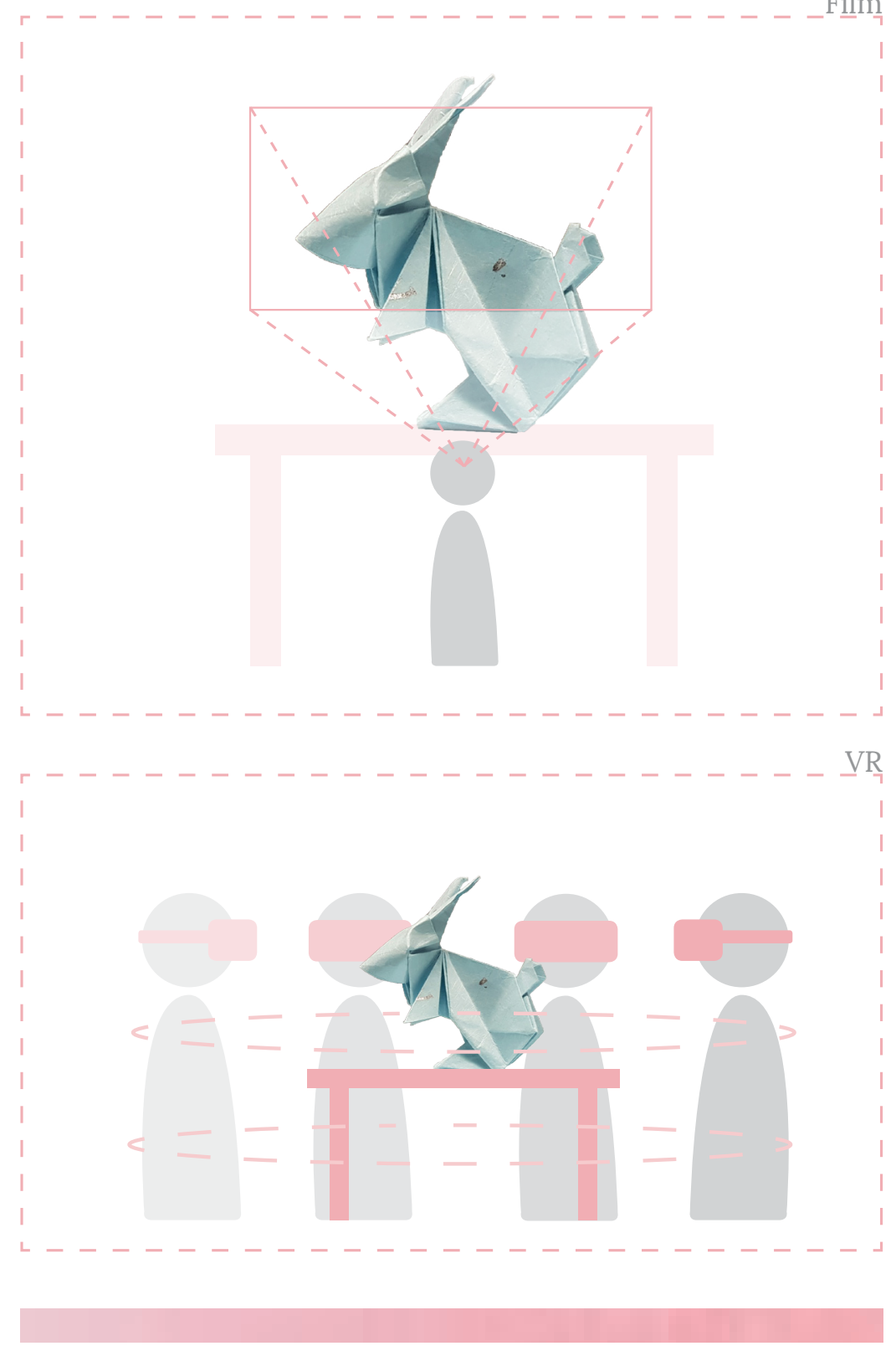

Fig.03. Diagram showcasing the framed narrative of film in comparison to VR where the user have their own agency and freedom of movement 
Physical modeling has been used in architecture for centuries but it was not until the Bauhaus period that it became introduced as an official requirement in the architecture education curriculum (Morris, 2006). Smith (2004) observes how architecture models serves as a mechanism in which ideas can be tested and examined repeatedly. It is a process based method of re-examining the concept and showcase the architect's intent and thinking. Conceptual models are used as a method of actualizing sketch designs in a three dimensional form. Stanford Hohauser (as cited in Smith, 2004) describes architecture models as the easiest presentational techniques to be understood by architects and clients alike. Architects can think spatially while clients do not have the same training in order to do so, thus models are an effective way of showcasing the design than two dimensional architectural drawings. Due to cost, time constraints and the effectiveness of digital modeling, physical models have diminished in importance.

In saying so, physical model has a great range of benefits in comparison to their digital counterparts. Materiality and craftsmanship are an example of this. The concept of trial and error and the accidents in the process of design (through interaction of lights and gravity) help produce new iterations of models (Morris, 2006 \& Cheng, 1995). This process of creating celebrates the mode of hand crafting and the imperfection that naturally occurs with the human element (Morris, 2006).

"There's a basic understanding that crafting something by hand out of wax, clay, wood or metal is a performance in itself, and one that involves the collaboration of the artisan and their chosen material. Software and digital fabrication processes are capable of producing intricate and beautiful things, but the method through which they came into being is so diffuse that it's hard to still read a personal experience in that object." (Openshaw, 2015, p. 9)

The constant experimentation during physical modeling allows us to learn the limits and potential to fully understand the material. This collaboration process of the creator and materiality enhance the ability to create meaningful models that emotionally connects to the individual (Pallasmaa, 2009). 

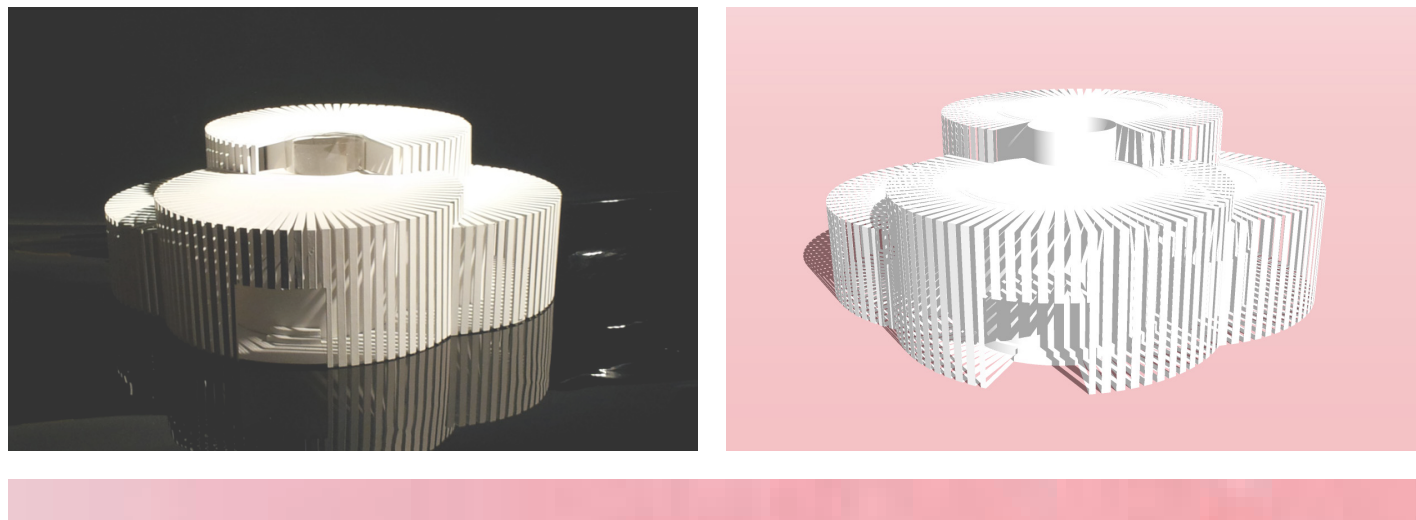

Jakimowicz (2002) argues that the designer is separated from the model through the use of the digital domain. I believe there is truth in this statement as we are creating digital models through various clicks of the buttons and not engaging fully as we would with a physical model. However, there is merit in digital software allowing the freedom of creating complex geometries. But with this freedom, the understanding of material properties and assembly processes are diminishing in the post-digital age (Cheng, 1995). Computer programs do offer the ability of texture mapping onto their geometries, which allows users to replicate real life materials onto the digital form. But with the compulsion for photorealism, critics have argued that the abstraction present in the conceptual phase of design is lost (Morris, 2006).

There is a perfect, clean and untouched aesthetic about digital models. Morris (2006) notes how the aesthetic is "matched by the perfection of its means of production" (p. 169). This 'perfect' aesthetic of virtual models is another step towards relinquishing the abstraction in design in order to represent the real. In losing the abstraction, the digital models appear too 'finished' with no room for outside interpretation. The rough physical model provides a level of abstraction and incompleteness that allows freedom of interpretation. Opportunity for alternative design are available with the agency of the observer. 
The transition into the digital age means the necessity of designing with physical models is being re-evaluated. In 2013 there was an experiment conducted by students from Osaka University exploring the differences in spatial understanding between physical and virtual models. Through the research, they have created both a physical and virtual model of a selected urban area in Japan. They asked a variety of individuals questions regarding height comparison of the buildings, estimation of the actual height of a building and scale of the physical model. The physical model was positioned at the same angle as the digital model on the computer screen in order to limit the difference in medium (fig.05). The final results were heavily favoured towards the physical model as it produced higher level of accuracy with the estimation of size in comparison to the digital model. (Sun, Fukuda, Tokuhara and Yabuki, 2014)

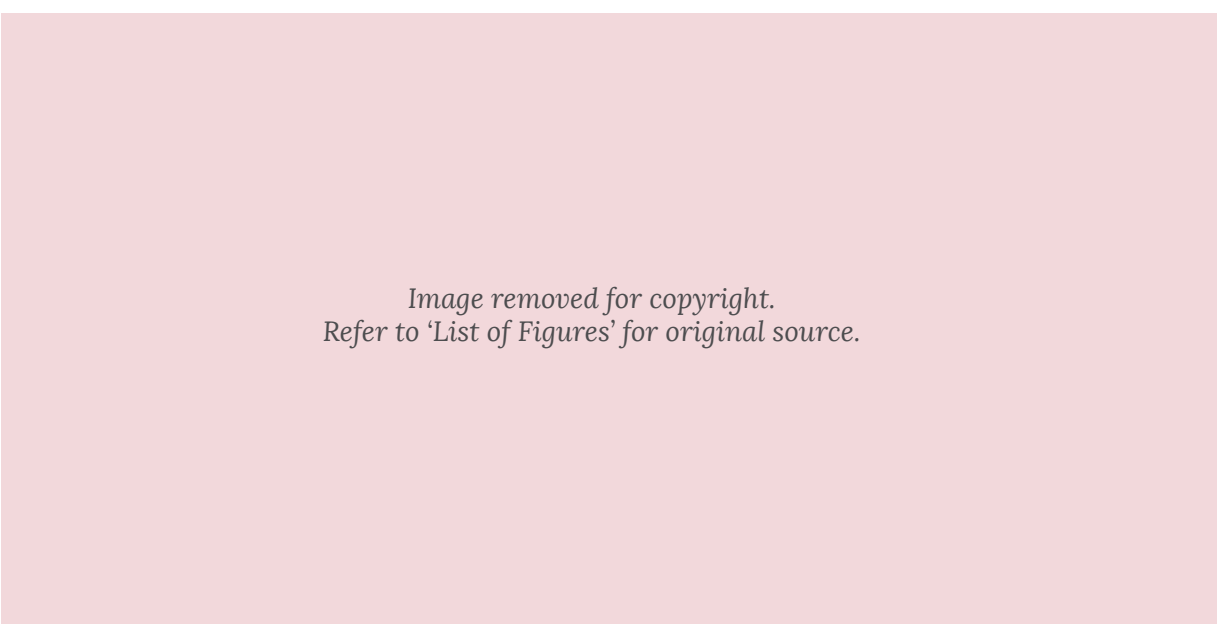

Fig.05. Diagram of the experiement conducted by Osaka University Students, Sun, Fukuda, Tokuhara E Yabuki, 2014 
Physical models are still an important designing tool based on the results of this study. I find this experimentation provides an interesting look into the spatial awareness of physical and digital models. However, I think there is a disadvantage of comparing the two in this medium. The physical model is a three dimensional representation while the digital model is being judged on the screen which in essence is a two dimensional representation. "Physical models communicate better than their flat screen simulations because sculptural objects inhabit the real space of the viewer, while frames isolate imaginary space." (Cheng 1995 p. 303) This sentiment is echoed by Stavric, Sidanin and Tepavcevic (2013). Thus, an accurate mode of comparison would be to use VR in order to judge the digital models.

Though there are many advantages and disadvantages of both physical and digital models, there is an opportunity for the two methods of designing to merge. Hybrid design techniques are available such as scanning and photo digitisation methods (Hadjri, 2003). This will allow the translation of physical models in the digital realm. I have taken the studies and theoretical understanding about both forms of modeling and apply it through my thesis research. Using both the advantages of physical and digital design I aim to minimise the gap between the two mediums. 


\section{Chapter 3:}

Case Studies 
The previous chapter discussed the 'research for design' component of the thesis, in this chapter the precedents which influenced the visual and design aspects of the research are covered. It explores precedents from across the gaming, film and performing arts industry with their relations to architecture. They are chosen to showcase the relevancy to my design research and are key elements in informing the direction of my design process.

Choosing to explore case studies outside the discipline have broadened my understanding of alternate ways in designing architectural spaces beyond the conventional methods. Materials, colour, aural, lighting and cues used in these precedents have provided new range of characteristics to explore with my own design. 
Image removed for copyright.

Refer to 'List of Figures' for original source.

Fig.06. Scaled set of The Grand Budapest Hotel 
Developer: State of Play

Release Date: 3 December 2014

Type: Interactive Media

Lumino City is a handmade puzzle solving interactive media which combine the use of real-life filming with digital animation. The main feature and dra of this game is the environment which in itself is an intricate architectur piece. State of Play directors; Katherine Bidwell and Luke Whittaker hired architect, Catrina Stewart to help in the designing and developing their ambitious environment (Cameron, 2015). This game creates a worl built with paper and cardboard models (that inherently became a scale architecture model) instead of the standard digital polygon. The richnes of using real material is apparent in the game as it sets a warm tone the is usually lost with just digital animation. "Models appeal to the emotion through the tactility of materials, the miniaturization of scale, and the mar of the human hand" (Cheng, 1995, p. 304). This enhances the experience grounding the seemingly "virtual" space to allow for the physicality of th material to be the forefront of the design. This interactive media challenge the aesthetic and experience of standard virtual games and how usin lighting and real materials distract from the uncanny valley effect of virtu medium.

"There's such a sense of warmth you can give to a story using real materials - Luke Whittakers (Webster, 2014, para 5)

Reflection: I have used this case study as a visual and aesthetic precedent in helping me shape the direction of my project. The use of paper craft as building material to test and develop my research question came in hand as it is a resourceful material than can be used and manipulated easily. $A$ discussed by Whittakers in his IGF interview (Cameron, 2015), being a tea of one person partaking in this design research, it feels more achievab to make these elements by hand instead of attempting to replicate th aesthetic and mannerism of paper in a digital form. 
Image removed for copyright.

Refer to 'List of Figures' for original source.

Image removed for copyright.

Refer to 'List of Figures' for original source.
Image removed for copyright.

Refer to 'List of Figures' for original source.

draw

cture

akers

ng of

vorld

caled

iness

that

tions

mark

ce of

of the

enges

using

irtual

ials."

lents

t as a

andy

ly. As

team

vable e the

Image removed for copyright.

Refer to 'List of Figures' for original source.
Image removed for copyright.

Refer to 'List of Figures' for original source.
Image removed for copyright.

Refer to 'List of Figures' for original source.

Fig.07. The environment of Lumino City 
Developer: Nyamyam

Release Date: 19 Feb 2014

Type: Interactive Media

Tengami is another puzzle solving interactive media using the aesthetic of a pop-up book to generate its interactivity and transitions. The entirety of this environment is created digitally but the developers have employed real world physics to ensure everything simulates reality. Nyamyam have opted to recreate everything by hand in order to test the theory. Although they have retained the material properties of paper, one can tell it is a digital constructed game evident by the clean and crisp environment. The colour palette throughout the game is bright and eye catching contributing to the stunning presentation of the game. Aural cues and sound effects also added an extra depth to the interactivity and immersion qualities present. In combination with the beautiful soundtrack it sets the serene quality intended for the end result.

Reflection: I have looked at this interactive media as a contrast to Lumino City. Here is a game that is mimicking real life material of paper and the aesthetic of a pop up book but is all made digitally. It is a beautifully-made media but the contrast is apparent when compared to an environment that is made entirely by hand. The physical materiality and warmth is lost through the imitation of real life material. Sound effects were used sparsely throughout the game but the audio cues helped in immersion of the game. I have taken this into account in my design. Another key factor was the movement of the player throughout the medium. The player moved through each scene at a slow walking pace allowing the viewers to absorb and appreciate the environment. The idea of limiting speed was a useful component moving into my design research. 
Image removed for copyright.

Refer to 'List of Figures' for original source.
Image removed for copyright. Refer to 'List of Figures' for original source.
Image removed for copyright.

Refer to 'List of Figures' for original source.
Image removed for copyright.

Refer to 'List of Figures' for original source.
Image removed for copyright.

Refer to 'List of Figures' for original source.
Image removed for copyright.

Refer to 'List of Figures' for original source.

Fig.08. The environment of Tengami 
This animation studio specialized in stop motion animation and produce many hand crafted models as the set for their movies. They have mastered the blend between stop-motion and digital animation which brings on a unique aesthetic in the cinematic industry. The rawness and imperfection shown through the making of stop motion animation is the quality that makes this animation technique stand apart from CGI films.The real world materiality and lighting adds a tactile and human quality which is lost through the seemingly perfect aesthetic of digital animation. Although the majority of the material and models created for Laika Studio animation films are made with 3D printers and other digital manufacturing devices, the materiality present in the film are still rooted in the physical world.

Reflection: The beauty of the 'puppets' and sets of Laika Studio film shows the intricacy of scaled models and how they can come together to bring something amazing. The use of real materials as opposed to digital textures provide an interesting and unique end product. There is a roughness in real material properties that grounds the experience of the film. I have taken these characteristics and used it to aid in the direction of my research topic. Looking at this precedents as a successful blend of how the physical and digital can work together to create something great. 
Image removed for copyright.

Refer to 'List of Figures' for original source.
Image removed for copyright.

Refer to 'List of Figures' for original source.
Image removed for copyright.

Refer to 'List of Figures' for original source.
Image removed for copyright.

Refer to 'List of Figures' for original source.
Image removed for copyright.

Refer to 'List of Figures' for original source.
Image removed for copyright.

Refer to 'List of Figures' for original source.

Fig.09. 1-2: Images of the set of Box Trolls

Fig.10. 3: On the set of ParaNorman

Fig.11. 4-6: Images showing the sets of Kubo and the Two Strings 
Theatre uses a range of beautiful sets, masks, puppets and props to tie in their production of various plays. The colours and spatial qualities that can be derived from theatre sets I believe, can be beneficial to understanding how a space (essentially a box) can create many levels of interaction within it. The materiality and vibrancy of the sets combined with props, music and performance ties the production together to give the audience an immersive experience. Theatre sets invites a level of abstraction and imagination through their design as majority of the sets composed of minimum furniture, painted or digitally projected backdrops, and prop elements placed in the foreground. The power of the 'implied space' allows audience to see entire rooms and landscape with only the fundamentals.

Reflection: Theatre in contrast to film does not inherently have a set focus frame controlling exactly what the audience should see. They have subtle cues in the form of sound, lights and movement in order to redirect audiences' attention to important aspects of the production. I think this quality will be useful in creating a virtual environment in VR as the virtual space is one that the participant can freely explore without frame restriction. Users can be guided through the environment using cues prevalent in theatre production in order to explore the surroundings as the creator intended. 
Image removed for copyright.

Refer to 'List of Figures' for original source.
Image removed for copyright.

Refer to 'List of Figures' for original source.

Image removed for copyright.

Refer to 'List of Figures' for original source.

Image removed for copyright.

Refer to 'List of Figures' for original source.

Image removed for copyright.

Refer to 'List of Figures' for original source.

Fig.12. 1: Matilda the Musical, Rob Howell (Designer), 2012

Fig.13. 2: The King and I, Michael Yeargan (Designer), 2015

Fig.14. 3: American Idiot, Christine Jones (Designer), 2010

Fig.15. 4: Martha, Linda Leon Art, 2013

Fig.16. 5: Sense and Sensibility, Great Lakes Scenic Studios, 2015 
"The best way to explain it is to do it."

- Lewis Carroll, Alice Adventure in Wonderland, 1865 


\section{Chapter 4:}

\section{Preliminary Design}


This chapter explains the process in which I have used to develop my research project. This exploration looked at paper and pattern with the goal of transferability from real material into the digital. The aesthetic of the experiment from the material chosen, the colours of the paper, and the construction method of the physical model were largely inspired by one of my case studies, Lumino City. These experiments and findings were all presented in the 1st design review (1/3 of the research project). The results of these exploration have expanded my knowledge and helped tighten the enquiries made in my research.

The narrative of Alice in Wonderland has been chosen as a base in which to test my findings and further explore the research topic. Being a universal and understandable fiction, observers do not need background information in order to understand and relate to the narrative. This story was chosen due to the fantastical and ambiguity elements of the novel. It allows limitless exploration and creation of environments that is not restricted by conventional standards. The paper aesthetic of my project fit within this narrative structure. With a pre existing narrative chosen, it allows me to focus on the material properties that is relevant to my thesis research. 

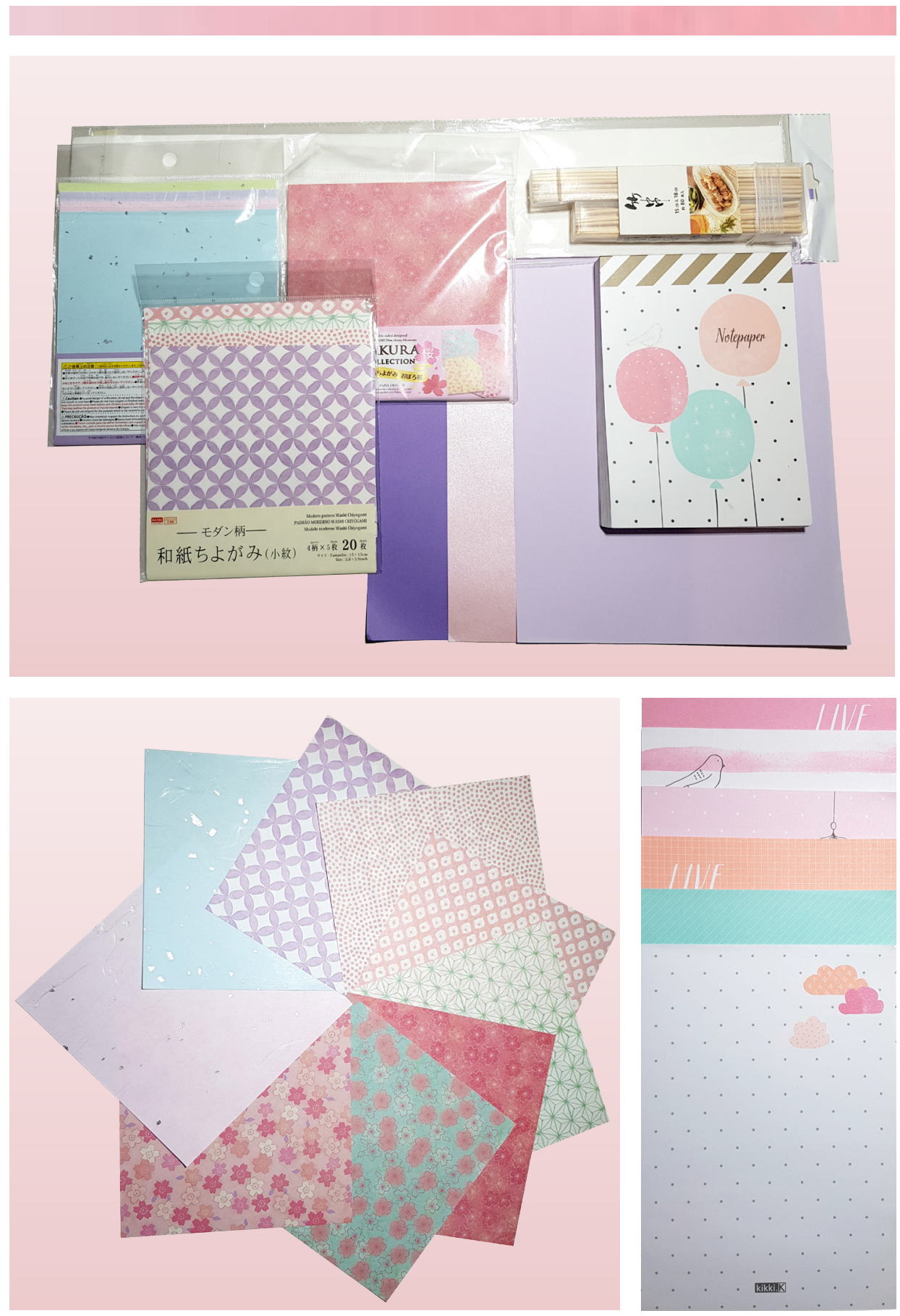

Fig.17. Different types of paper used throughout the design process 
What is Photogrammetry?

From the Oxford Dictionary, photogrammetry is defined as 'The use of photography in surveying and mapping to ascertain measurements between objects.' (Photogrammetry, n.d.). Through photographs, points and data are gathered to form an accurate reading in order to turn photographs of an object (or a subject) into a digital mesh. This technique is used to capture the 3 dimensional physical models and translate it into a digital model. 


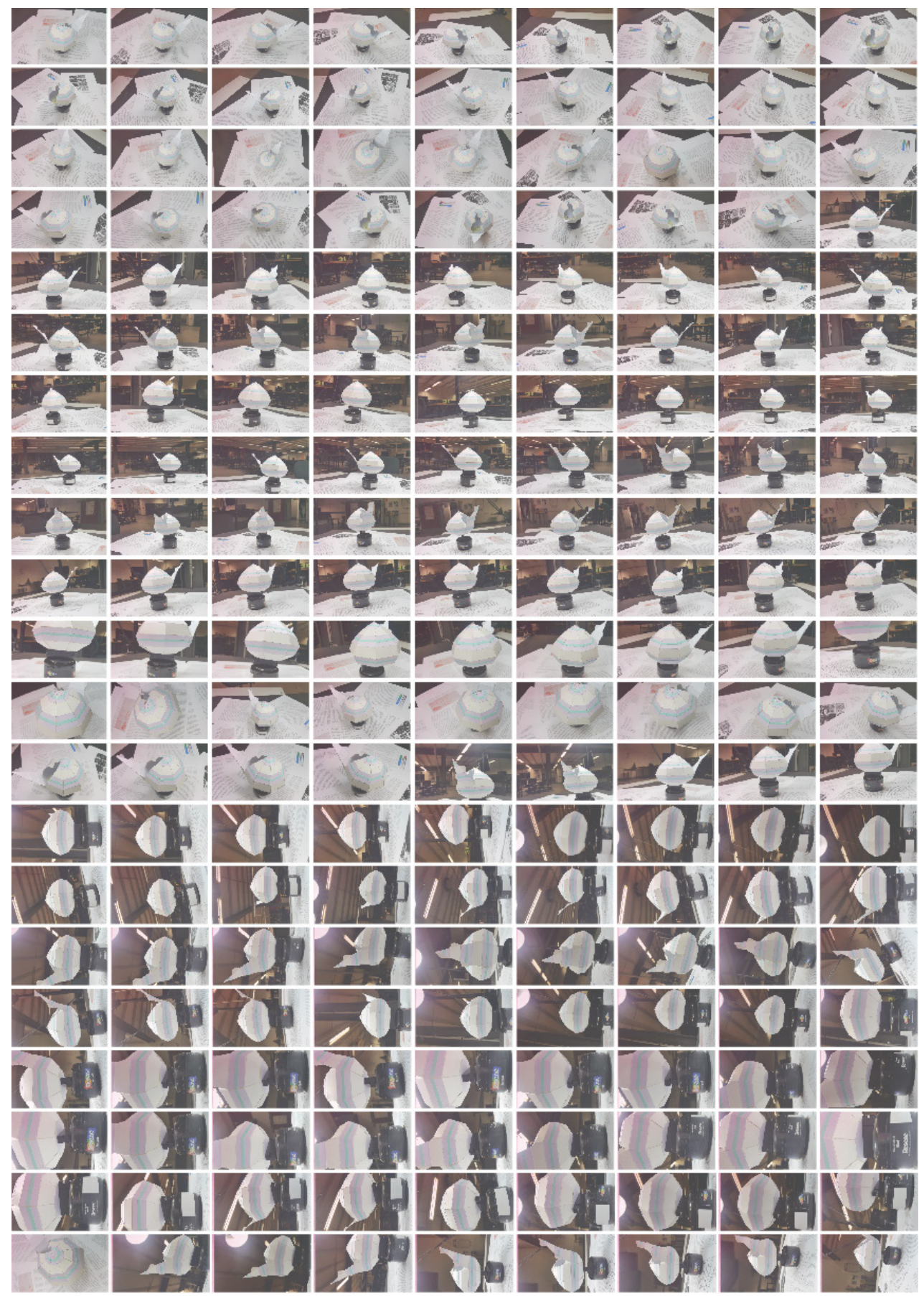

Fig.18. A range of photos needed to translate a physical model into digital mesh 
The first exploration started as a sandbox environment to examine how well paper texture could translate into the digital space. First, a small box is made out of cardboard with different coloured paper textures stuck on the outer surfaces. These boxes were glued and stacked together in order to create visual interest and depth. I have used the process of photogrammetry in order to transfer these physical models into a virtual environment. Two approaches of photogrammetry were tested, one with the entire model of stacking boxes and another with each individual component taken separately. This was done in order to see which method was the most successful in translating and manipulating the model in the virtual realm. These photos were then stitched together on a program called, Autodesk Remake which analysed the photographs and piecing the information available into a digital mesh. This mesh can be exported and imported into various format to be used in conjunction with other programs. 


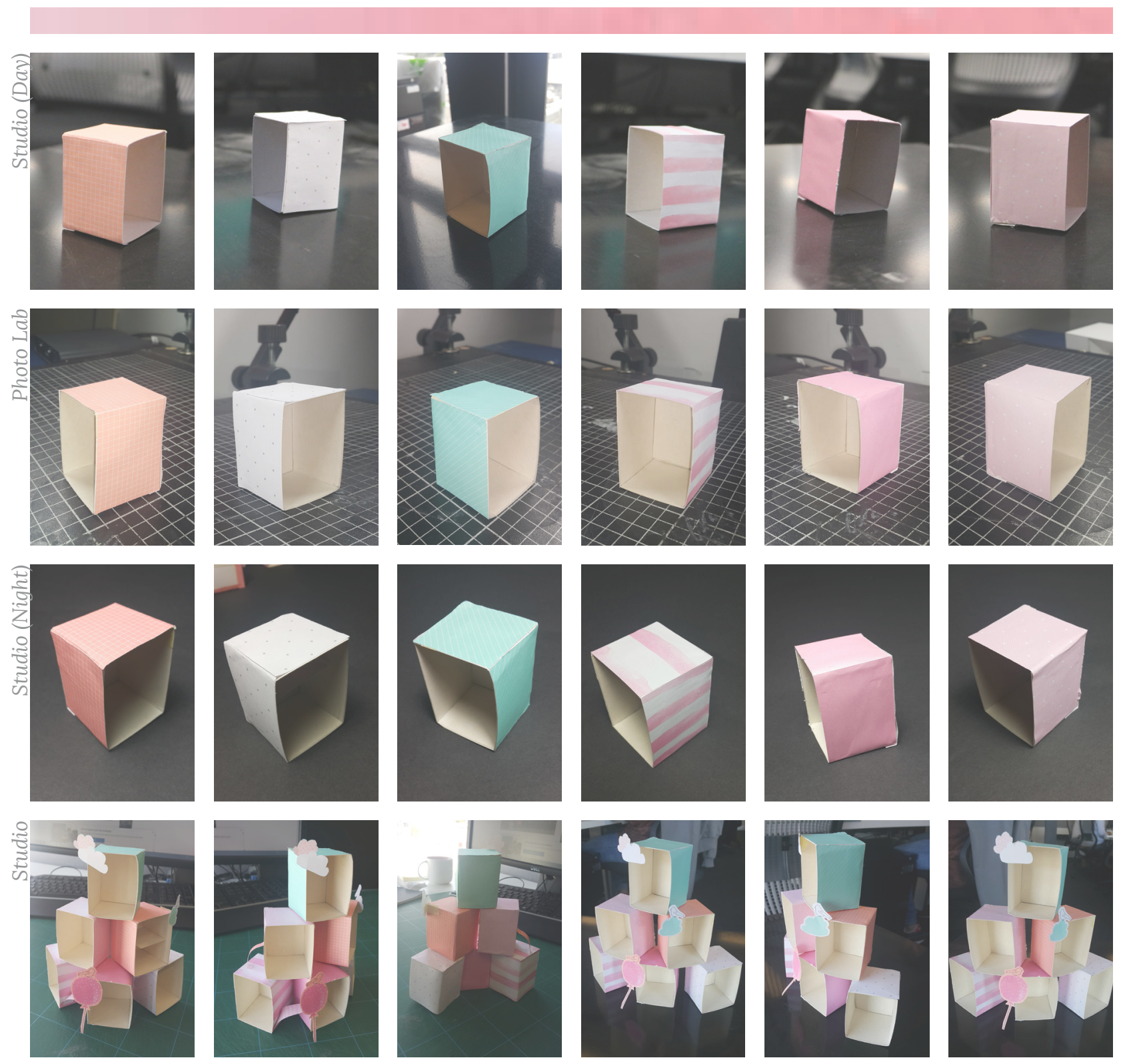

Fig.19. Photos taken for exploration 1 of the whole model vs the model as separate components. They are taken at different location and lighting environment 
There were a number of attempts that occured during my first few tests with photogrammetry. The work undertaken was incrementally more successful. The digital mesh produced were incomplete and had various holes perpetrating the mesh. This could be due to there being insufficient amount of photos, bad lighting or the paper not having enough texture for the programs to latch onto. However from these failed attempts, I've imported the models into Unity (a real-time virtual engine). This was to see how scale can change our perspective of space and having a person/ character exploring this in real time (game play) can be really effective instead of just still images. 

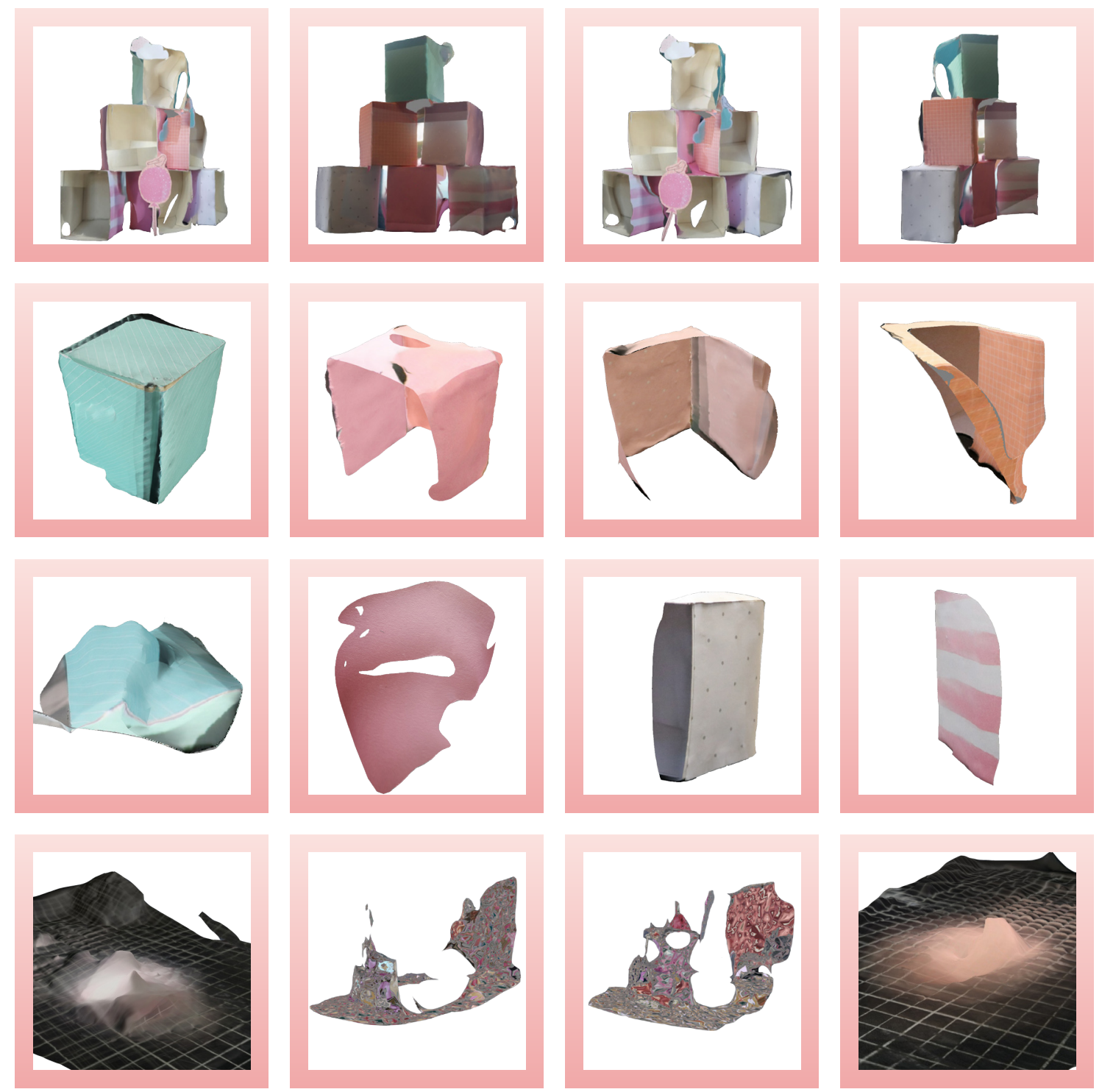

Fig.20. Photogrammetry outcome of exploration 1 featuring many failed attempts. The bottom row show glitches that appeared through some photogrammetry translation of their physical counterpart. 


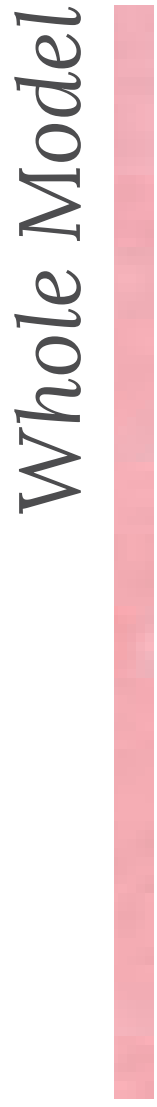

This first outcome is the digital mesh created from the physical model of all of the boxes. It produced a mesh which is an abstraction from the original but the materiality and pattern of the cardboard boxes are clear. Without any manipulation, the 'player' can explore the mesh as an obstacle with the 'holes' adding a unique touch to the digital model.
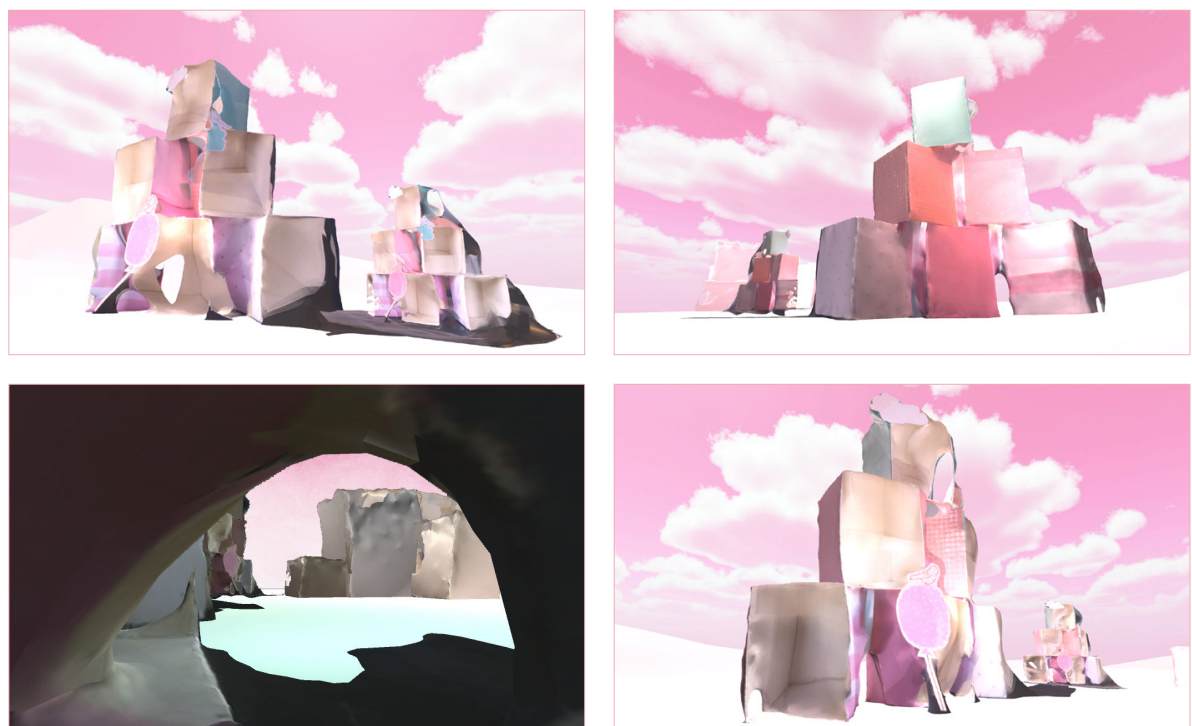

Fig.21. Screenshot of the photogrammetry model used in Unity 


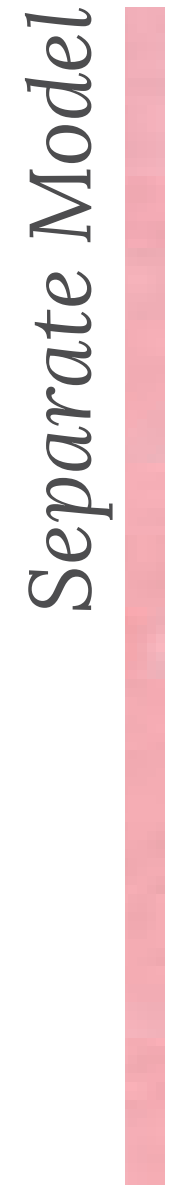

The second outcome is a digital model that has been created with separate components that have been individually photographed. The boxes being treated as separate elements provided a flexible outcome. This is due to the fact that there is unlimited ways for the single elements to be arranged as they can be duplicated, rotated and be manipulated with scale in order to create something unique.
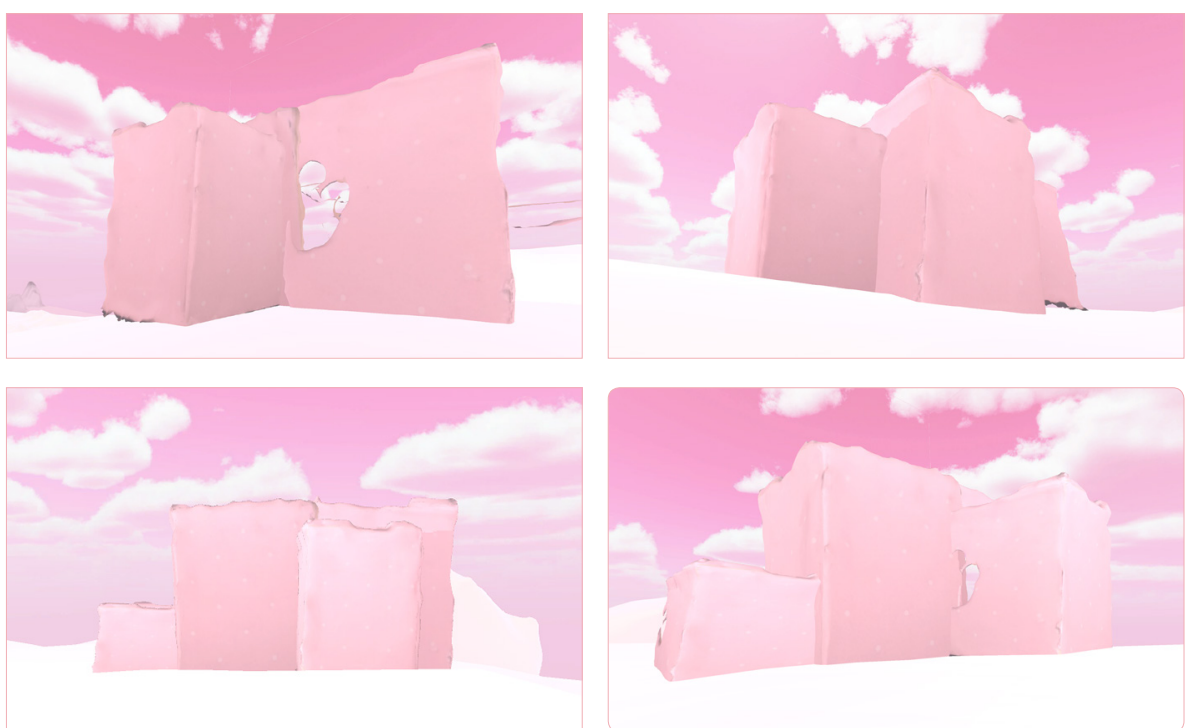

Fig.22. Screenshot of the photogrammetry model manipulated in Unity to create a different outcome 


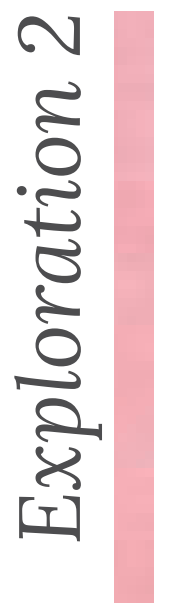

From this stage, I transitioned to another way of making paper models, this time: origami and templates. I have made different flower sets and tea cups in keeping with the theme of Alice in Wonderland. From there I once again experimented with photogrammetry techniques and lighting. However, there were many failures associated with the teacup no matter what location I have photographed them from. With further investigation, this was the result of the thinness of the paper used. The teacup was made with a template and thus the resulting model did not have any added depth. This means that while I was taking photos of the entire model, the program could not stitch together both sides of the teacup (inside and outside) as it was read as one surface. I have amended this by the simple act of turning the cup upside down and it was a success.

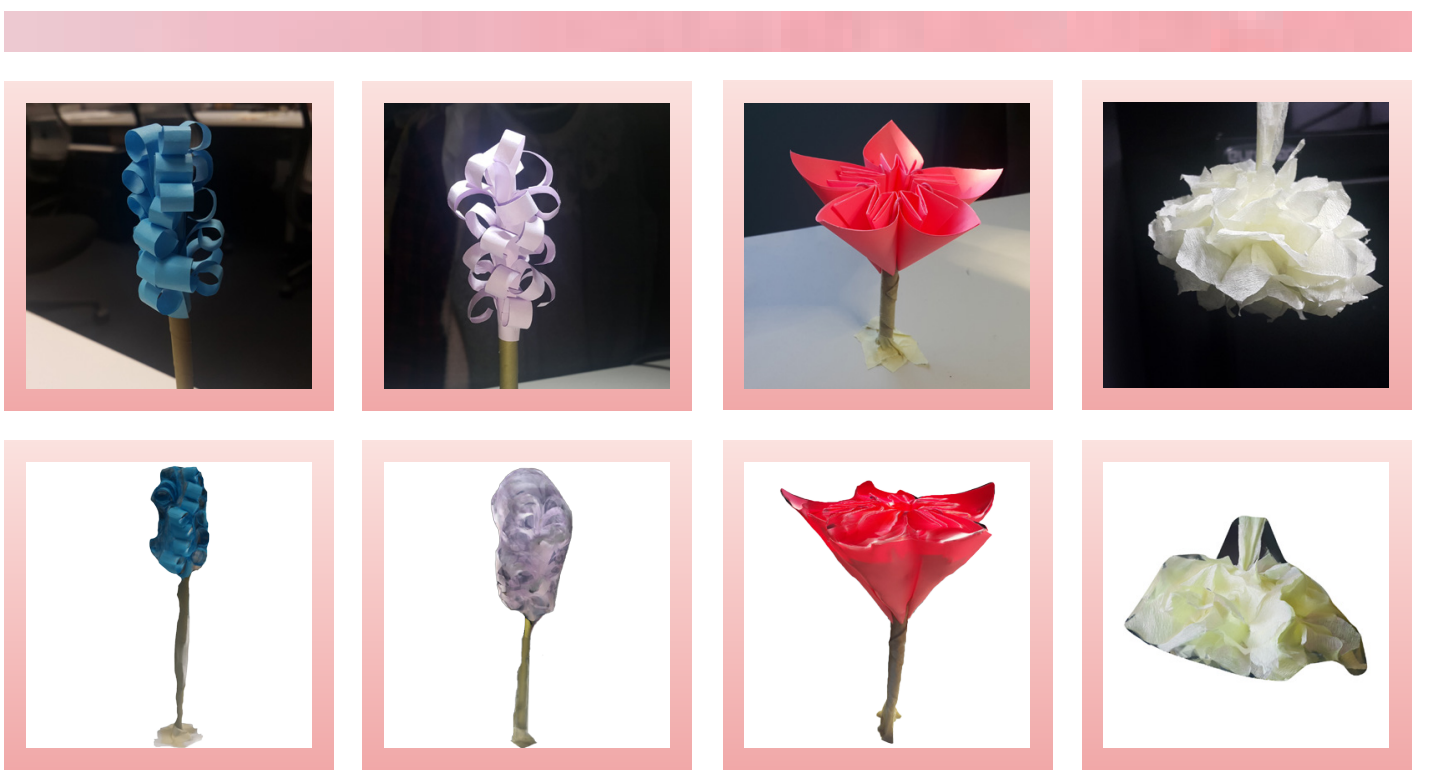

Fig.23. Physical model of the flowers made from various techniques and their photogrammetry counterpart 

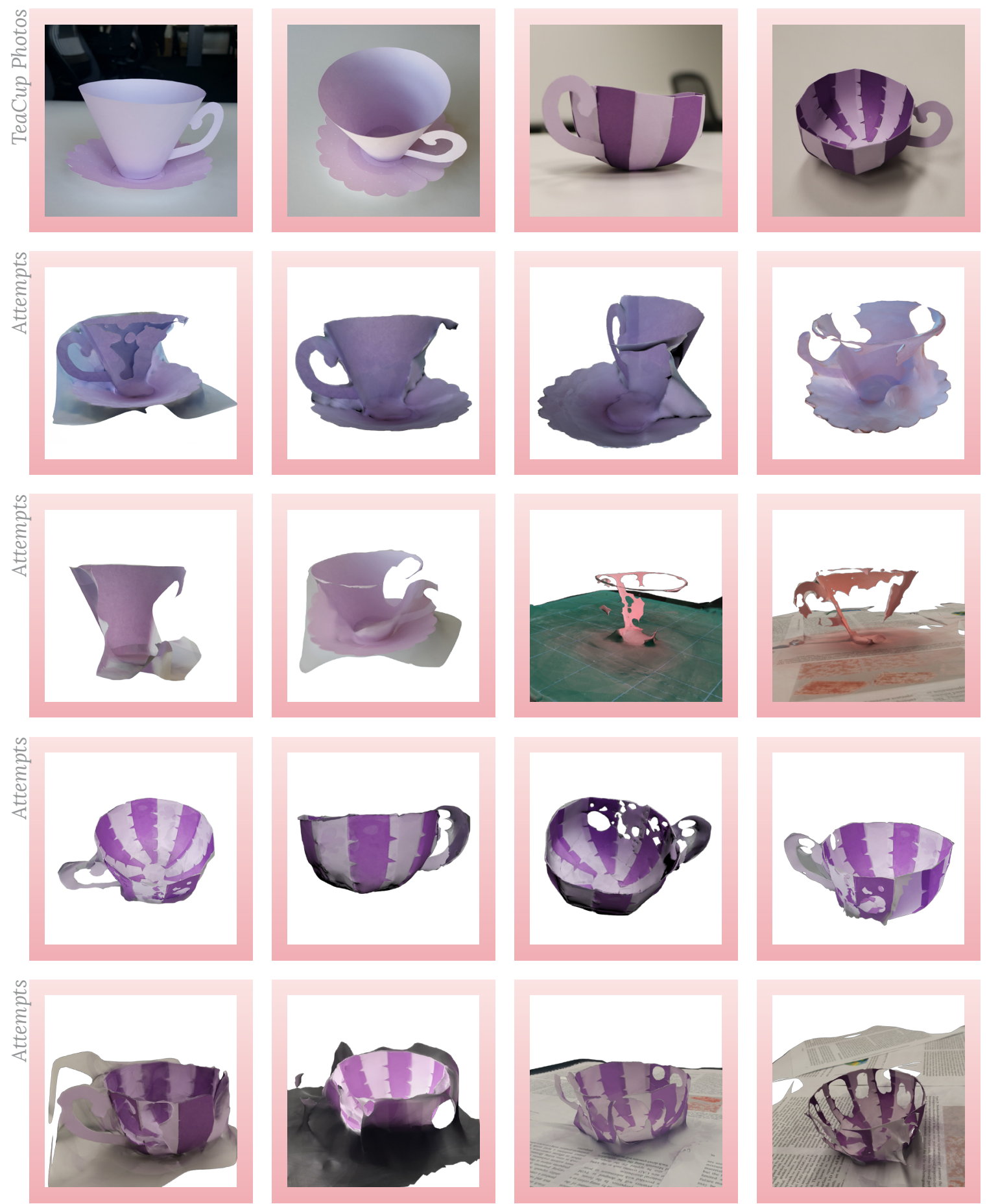

Fig.24. Teacup model photos (top row) and the many (16) different attempts producing failed results 


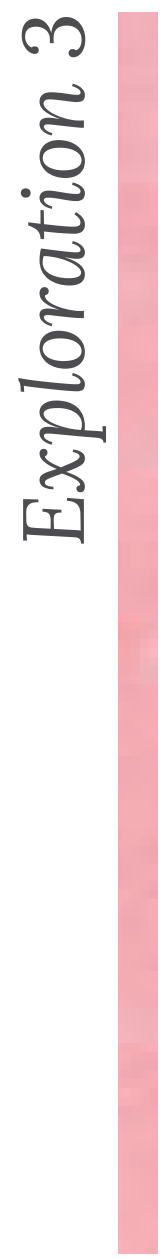

Another experiment was made by using a two dimensional shape or drawing cut out of paper. First I scanned the image in and placed them in Unity, however due to the 2D factor of the object, it appeared too flat and lifeless. I decided to test if it is possible to photogrammeterise a 2D element. From testing, the results were favourable and with the help of the photogrammetry program, thickness can be added to the digital mesh. This allows the object to appear like a 3 dimensional element in Unity.
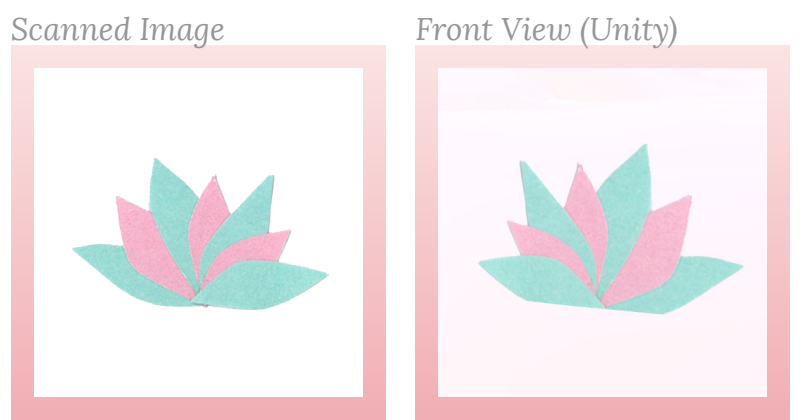

Side View (Unity)
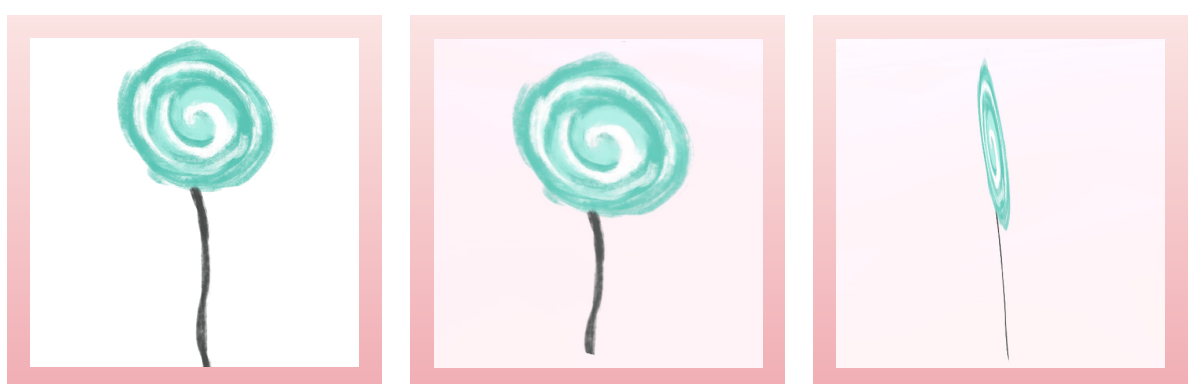

Fig.25. Scanned images translated into Unity produce flat 'objects' 

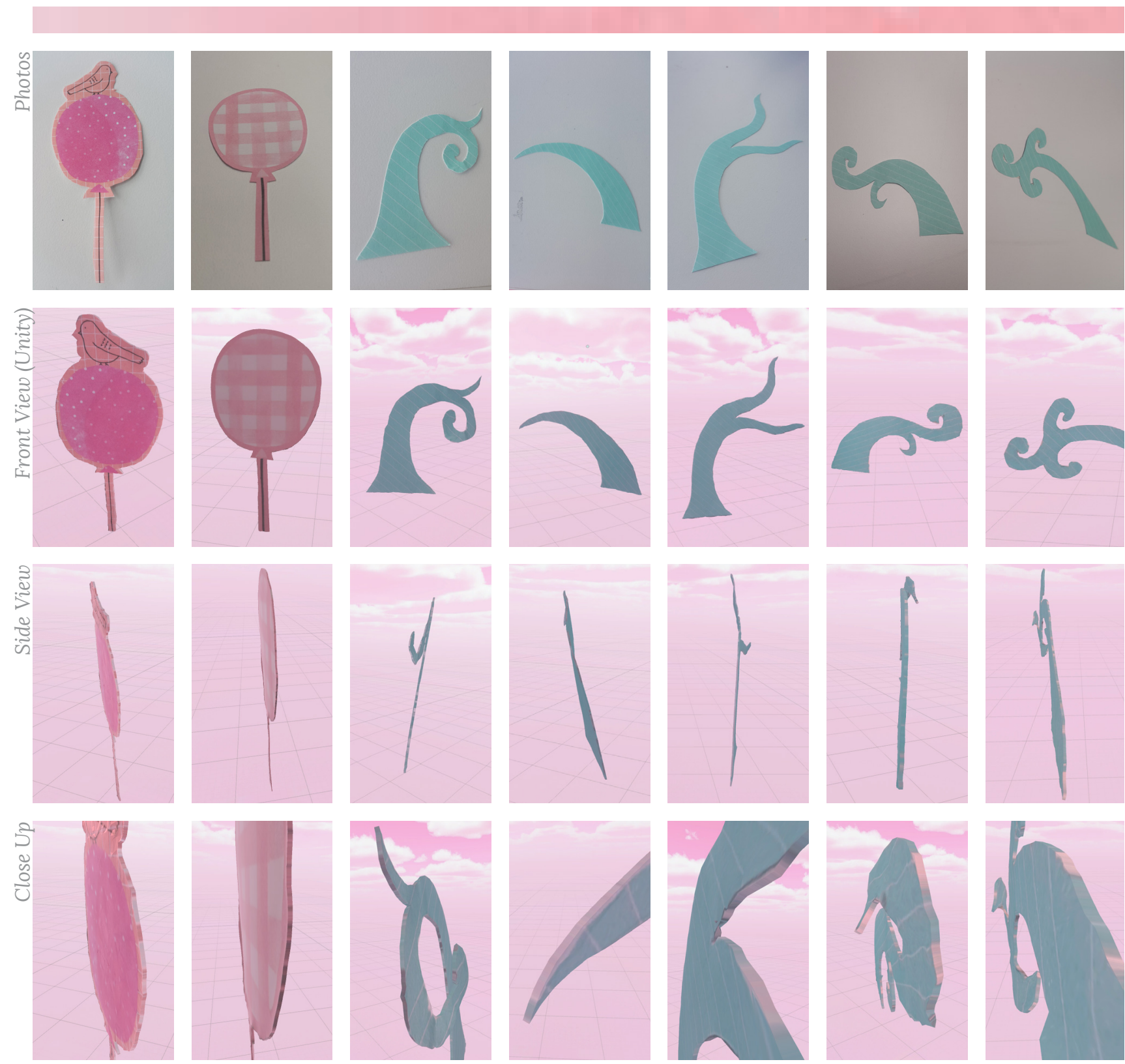

Fig.26. A different approach for translating 2D images into Unity 
The next stage was putting these individual elements into Unity to begin the process of building a virtual environment. I used the flowers and various paper models created and planted them on a landscape in the program in order to create a forest scene. The different elements worked together with the manipulation of scale in large quantity in the environment. As an interactive media, the elements places in the environment were stagnant and uninteresting. Therefore I learned to use the animation function on Unity in order to elevate the virtual space. This made a huge difference in creating an environment that was compelling to explore, bringing the virtual world to life.

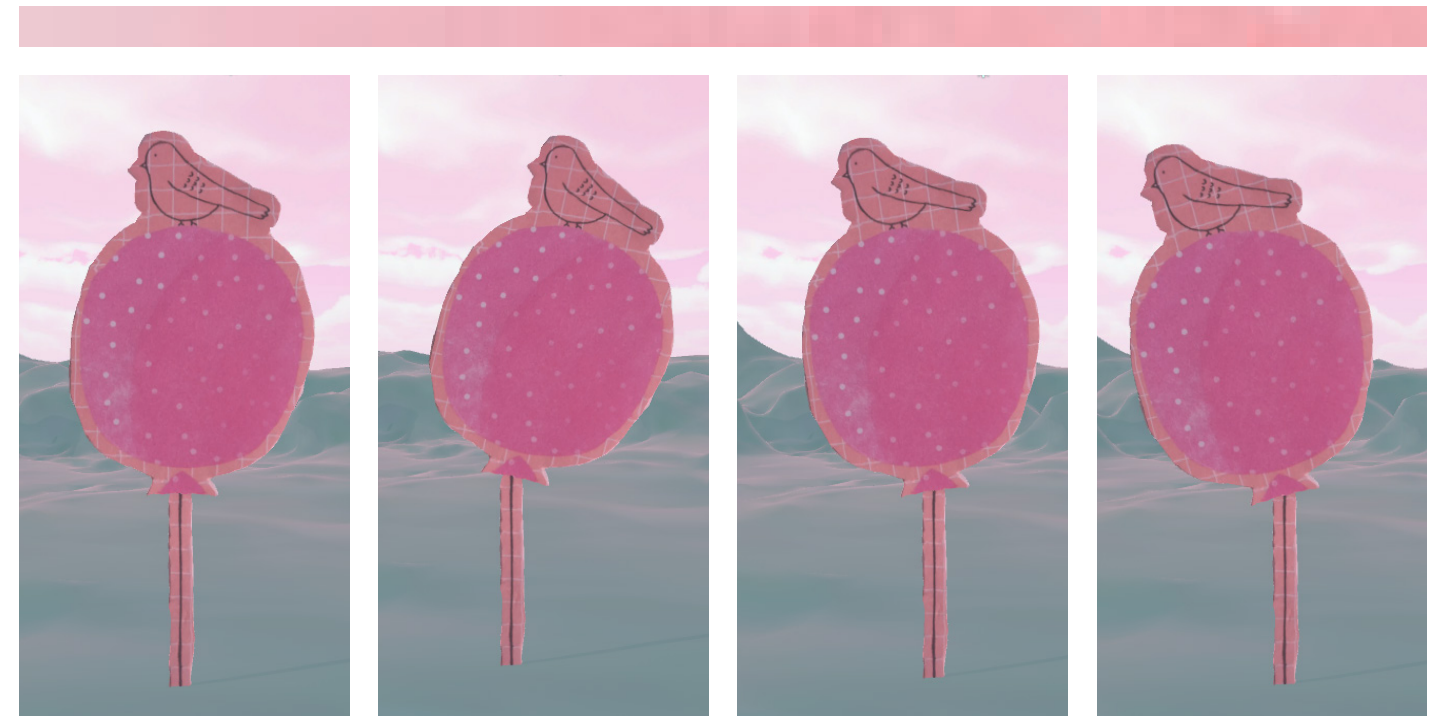

Fig.27.

Animation added to stationary object 

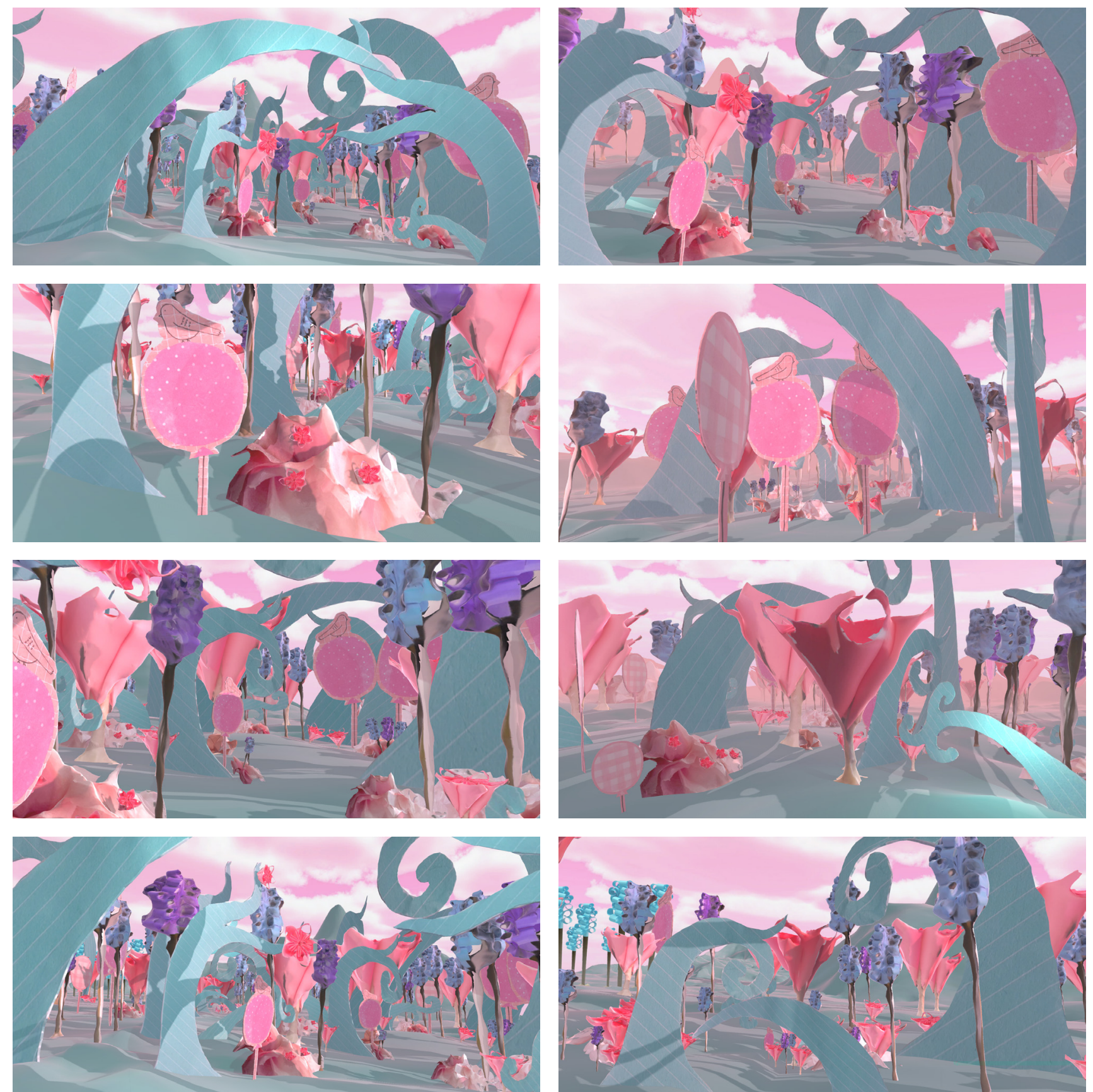

Fig.28. Screenshots of the environment created for the 3 month review using various elements created in exploration $1,2 \& 3$ 
As scale was another factor I was exploring, it was added and tested in the design. The feature allows the 'player' to enlarge and shrink while exploring the environment with selective elements changing size with them. This allows the player to experience the environment in three distinct scale; small - where the player is like an animal encroaching on the forest, medium - a habitable scale, and large - a scale where the elements were treated as scaled models. This help showcase the contrast in the material properties of the the 'objects' as a whole and how we read them depending on our scale in relation to them. Due to the small extent of the 'forest scene', walking through it in a human scale is short but immersive as the density created in the concentrated form bring life into the environment. When scale is reduced and the player is a miniature exploring the same environment, the forest now looks daunting as everything is larger than normal. This puts the player in a position not typically experienced on a human scale. Likewise, when the player is scaled upwards and is now considerably larger than the surroundings we start to see the environment as its own scale model that we can interpret from a 'God's eye view'. 

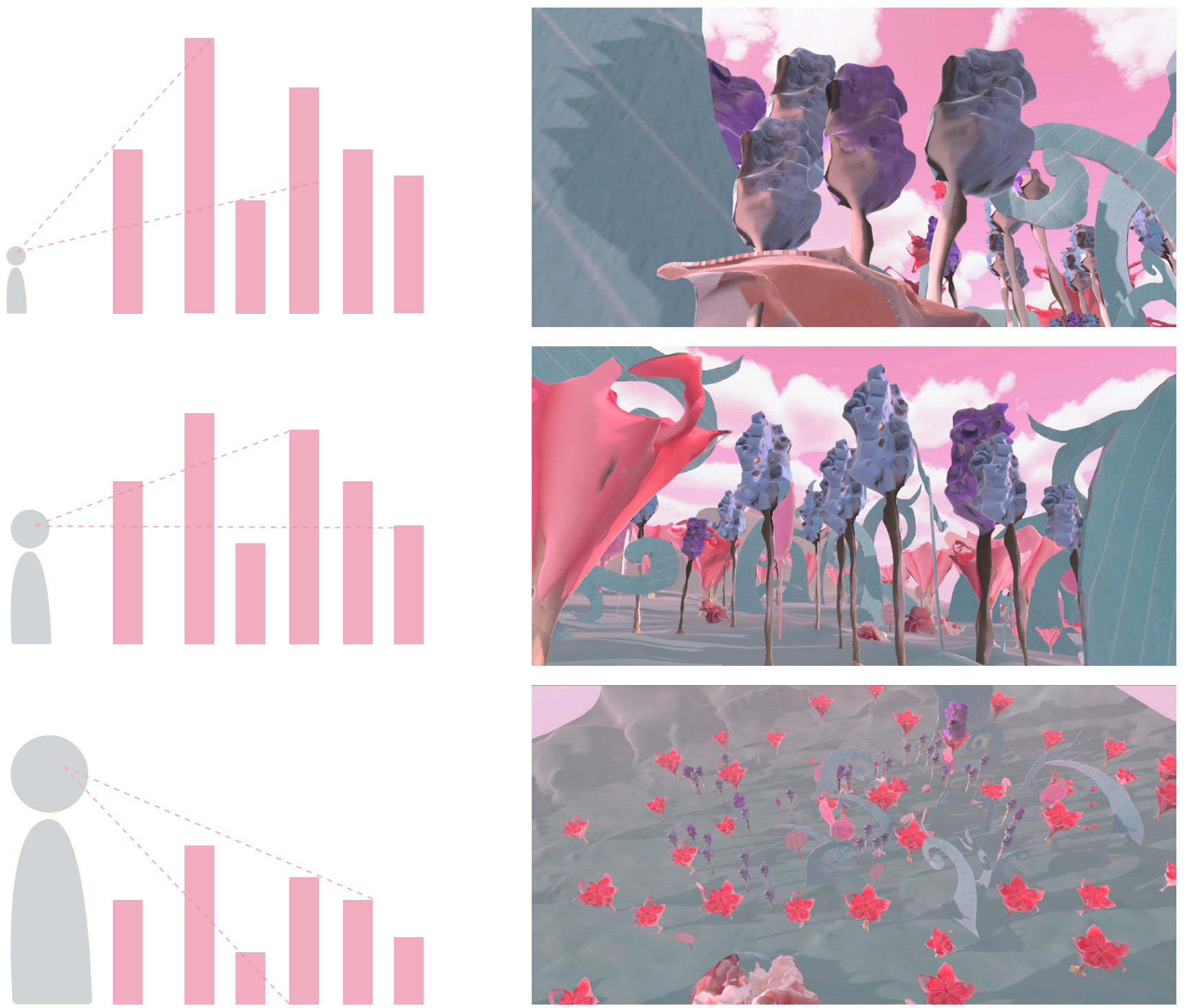

Fig.29. Diagram and screenshots illustrating the perspective of the user when scale is added to the environment 
From the explorations conducted in this chapter, the knowledge gained is important for furthering my design intent for this research topic. The understanding of photogrammetry; from the condition in which to take photos of a given object, to the texture and information provided from physical models and the object itself contribute to creating a workable digital mesh. Testing the difference between whole models and separate pieces allows the advantages of individual elements to emerge showcasing the flexibility during the creation process. Two dimensional and three dimensional objects provide a wider range of elements available in creating these unique spaces. Depth and contrast (of objects) elevate the nature of the immersive environment. The added bonus of animation and the ability to scale helped me understand the fundamental difference between the static image and the experience space. As the components are operating in a real time virtual environment (RTVE) the player's eyes are constantly readjusting to the moving objects in the surroundings which discourage turning the architectural objects into a linear abstraction. All the components of the explorations worked in focusing my scope and direction of my thesis topic. 


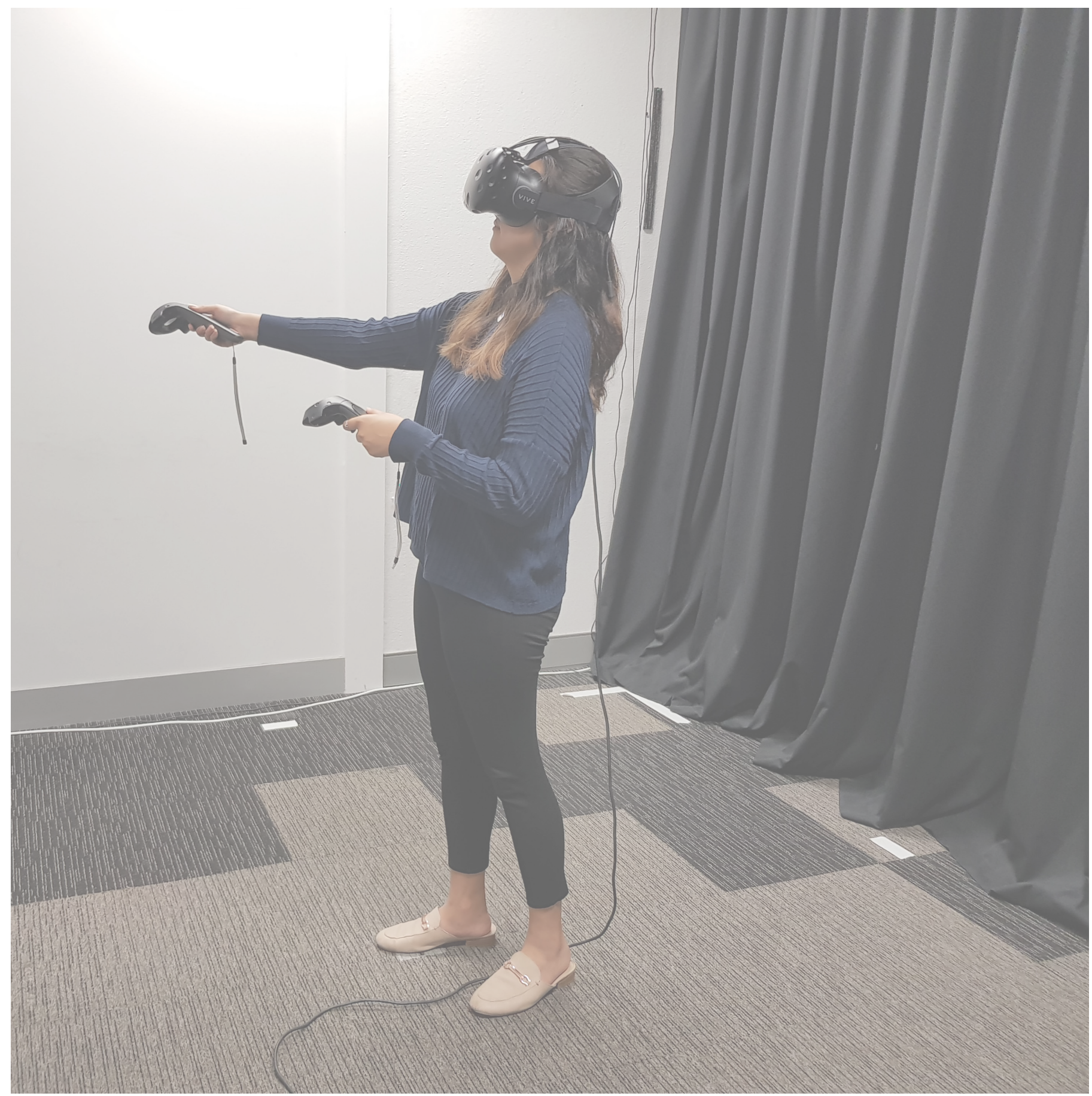

Fig.30. A student exploring the virtual environment using VR 


$$
\begin{aligned}
& \text { Chapter 5: } \\
& \text { Design Process }
\end{aligned}
$$


This chapter builds upon the knowledge gained from the previous chapter. The research question is answered and explored through a sequence of five different virtual environments which are based on the narrative of Alice in Wonderland. I have created five scenes that are interlinked to follow a narrative transition showcasing the contrast between a digitally made environment and environments made by hand. Each scene builds upon the other as the 'player' advances through each level, they gather new knowledge and 'ability' (e.g. increasing and decreasing in scale) that will enhance their exploration of the space.

Scene 1 - A digitally made room with digital paper textured applied.

Scene 2 - A rabbit hole in which the player falls down to enter the 'world' created by physical materials.

Scene 3 - Inside a teapot in which the player learns to use scale to advance to the next stage.

Scene 4 - A forest scene exploring the intricacies of the models made in the physical world through a range of scale and density.

Scene 5 - A physical model replica of scene 1.

The VR format has been chosen as the platform in which the environment is to be experienced. This is to allow a greater range of agency from the 'player' and not by a medium dictated by the designer seen through the digital screen or on paper. The VR experience is enhanced through the use of coding, animation, visual effects and sound allowing the participants to be fully immersive in the environment. 

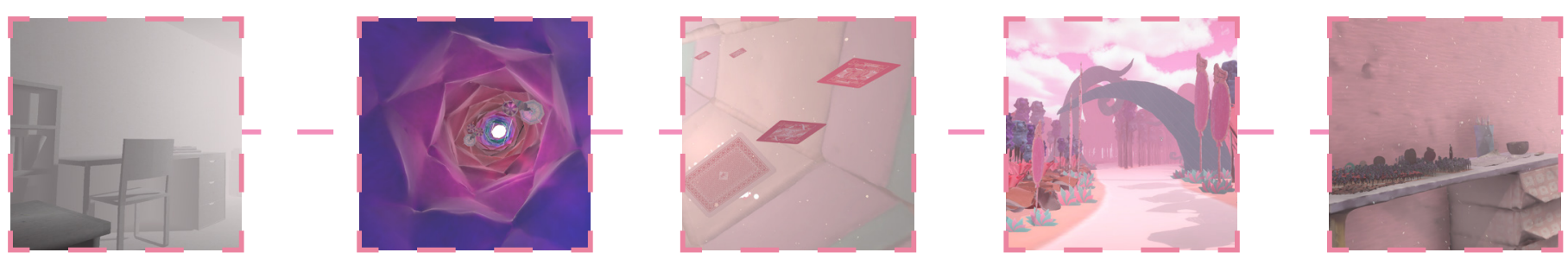


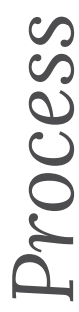
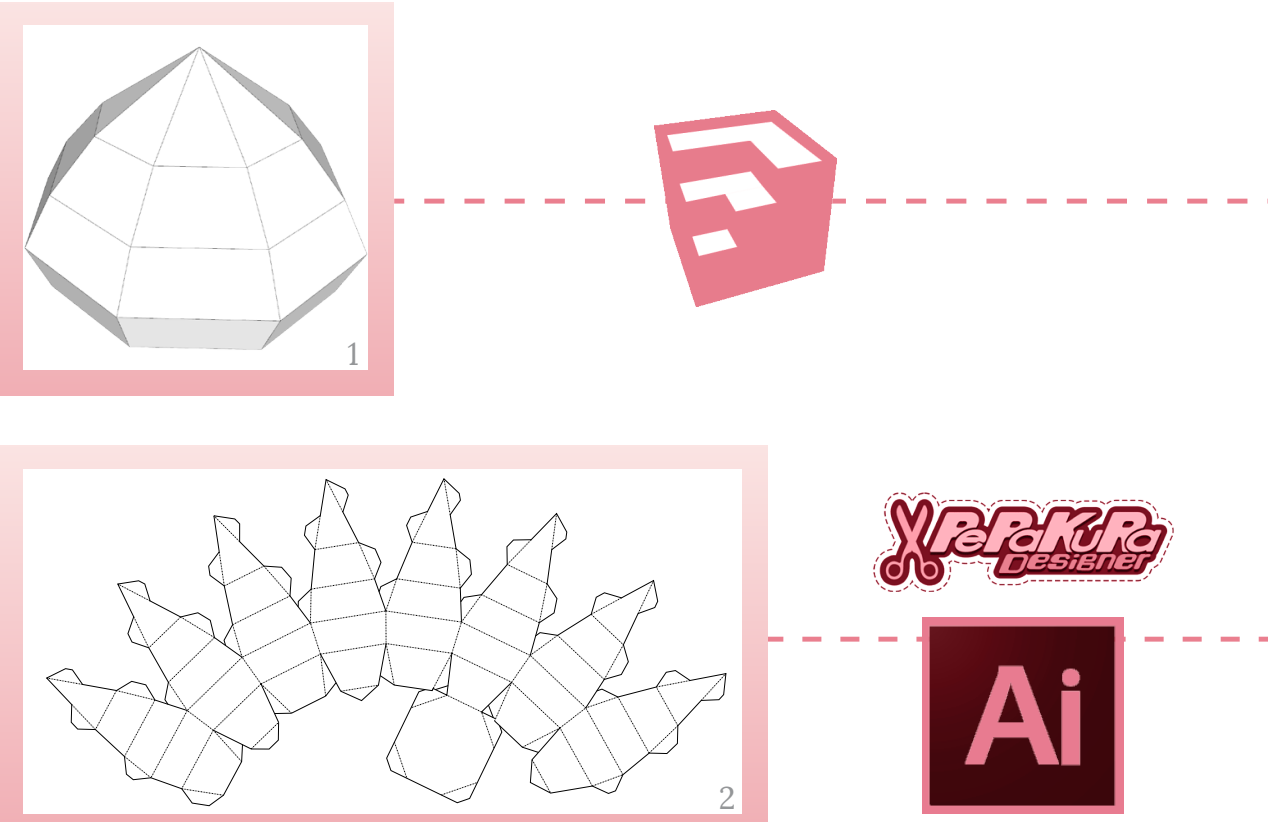

Miain
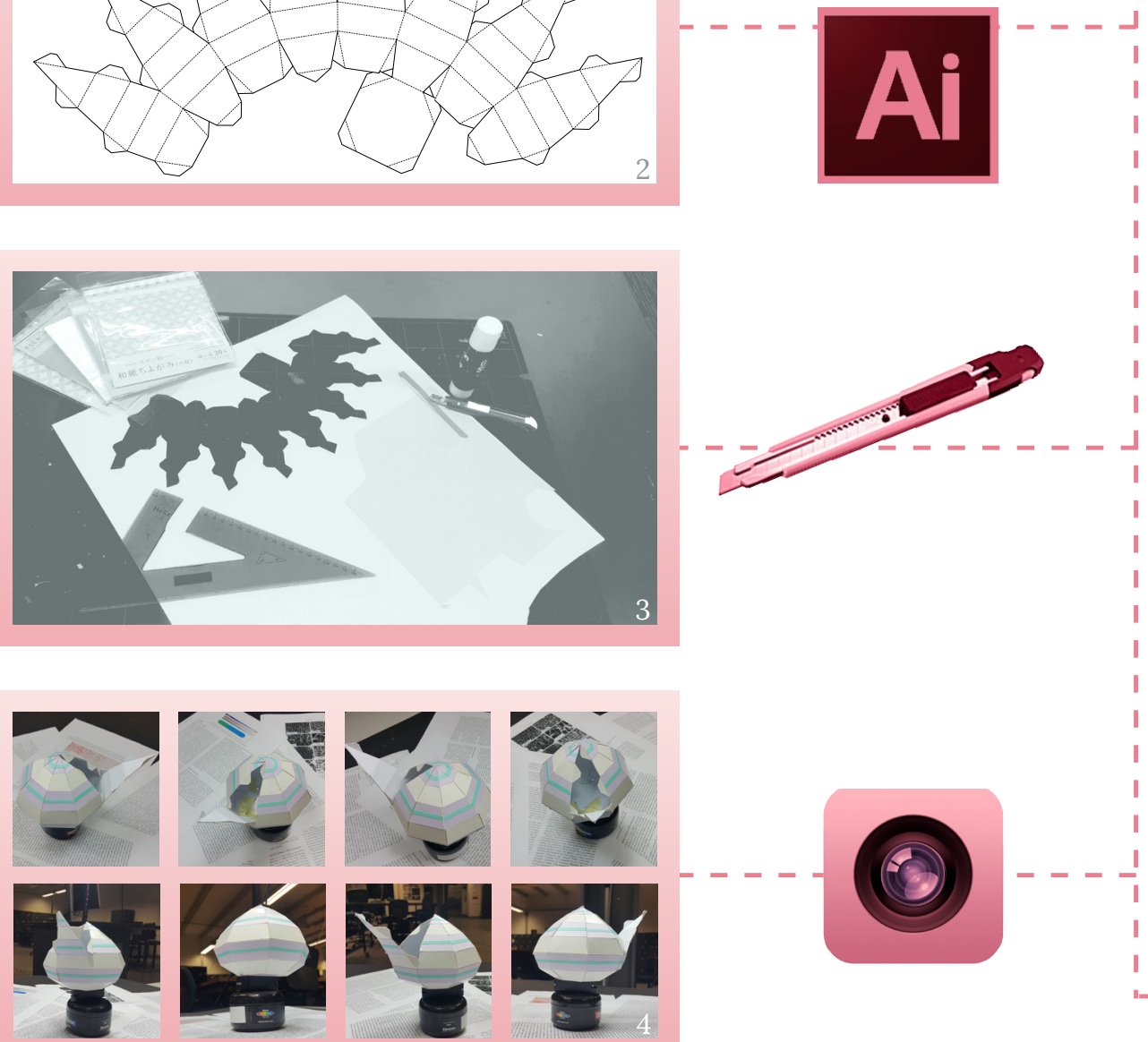


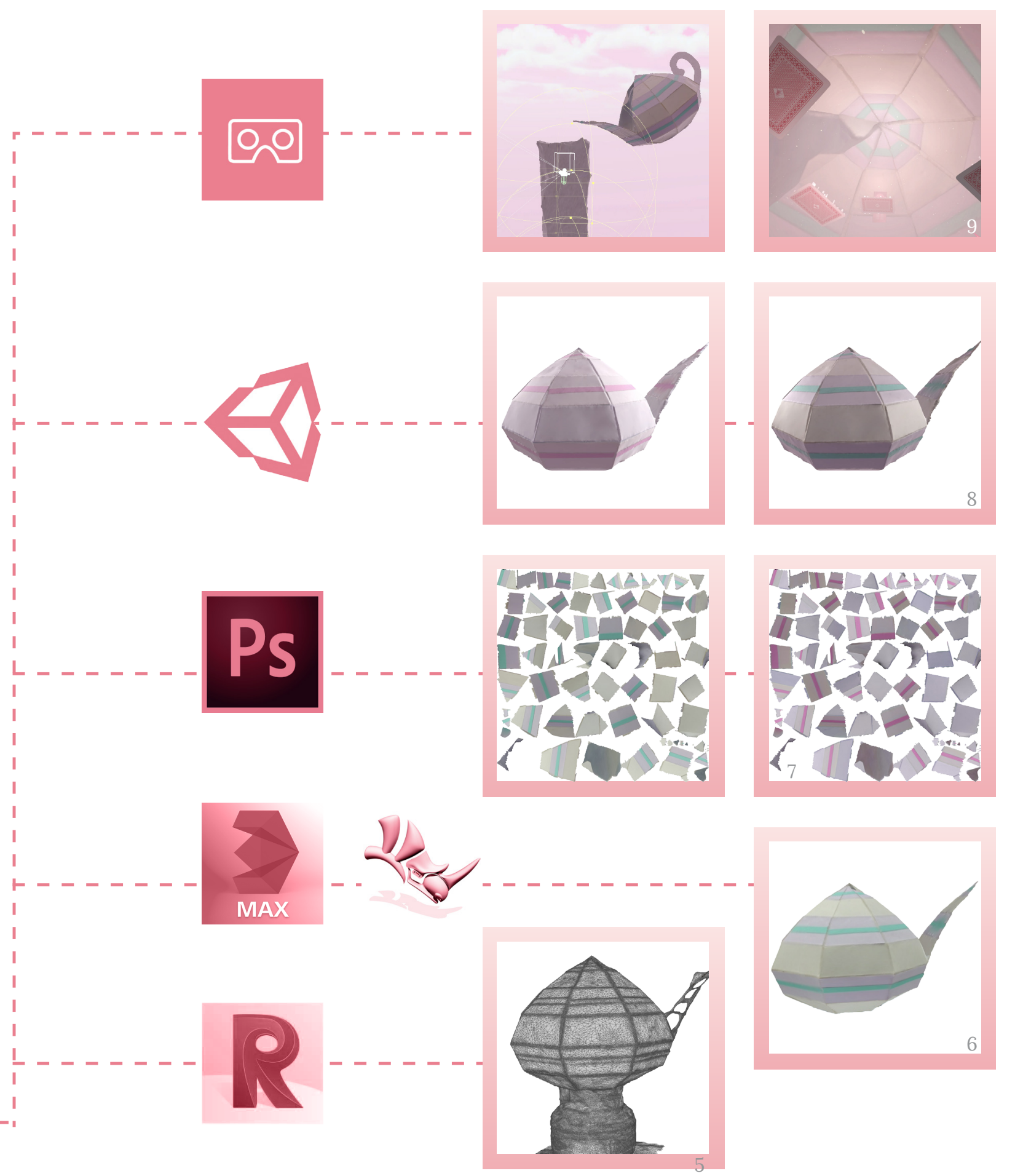

1) Model created in Sketchup

2) Digital mesh is turned into a net using Pepakura and modified with Adobe Illustrator

3) The model is then created using cardboard

4) Photos are taken of the model

5) Using Autodesk Remake, the photos are turned into a digital mesh using photogrammetry

6) The digital mesh is edited using Autodesk 3ds Max and Rhino

7) These are the textures that have been exported alongside the model, photoshop is used to modified the colour

8) Models are placed into Unity and a light source is added

9) A virtual environment is created around the object, which can be experienced with VR 

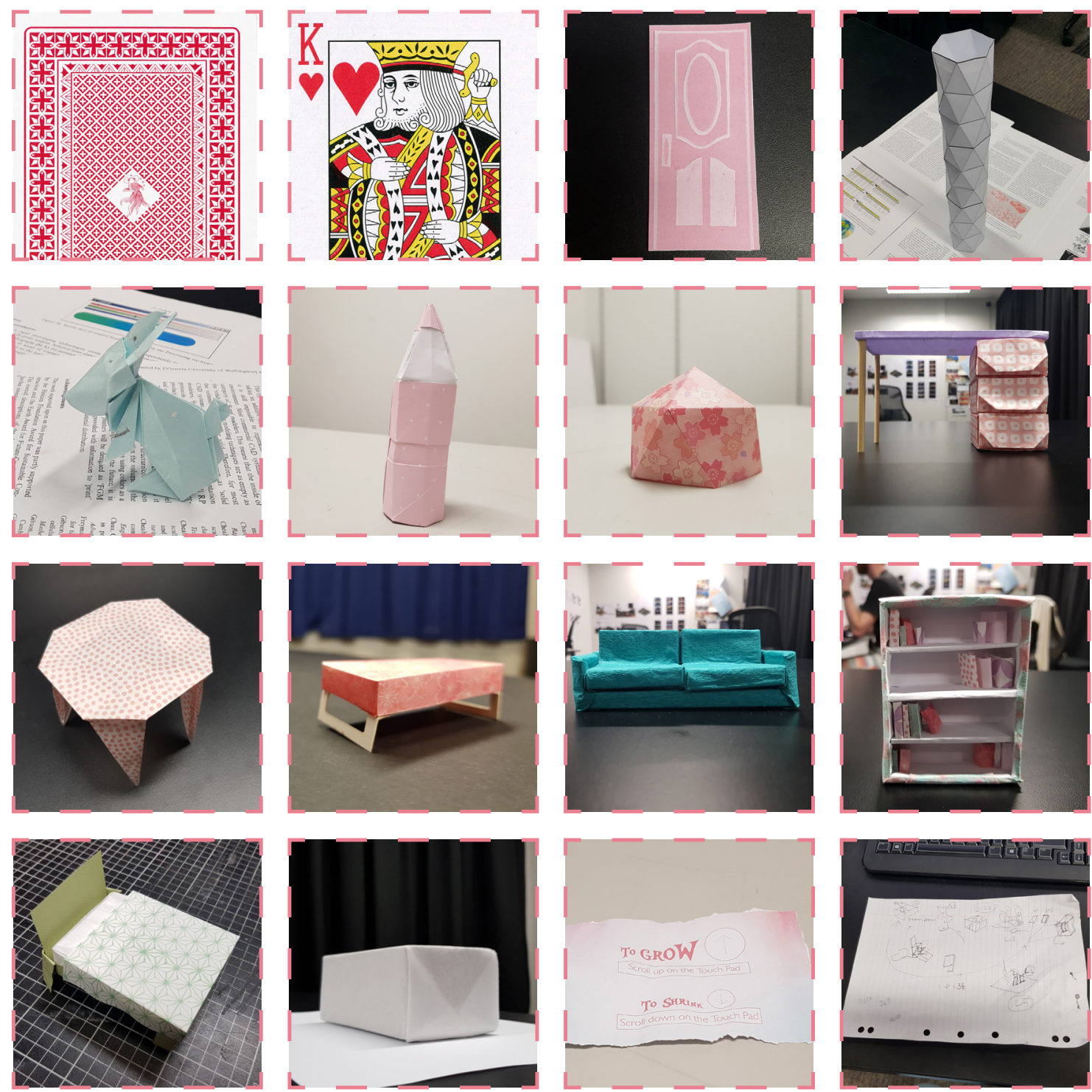

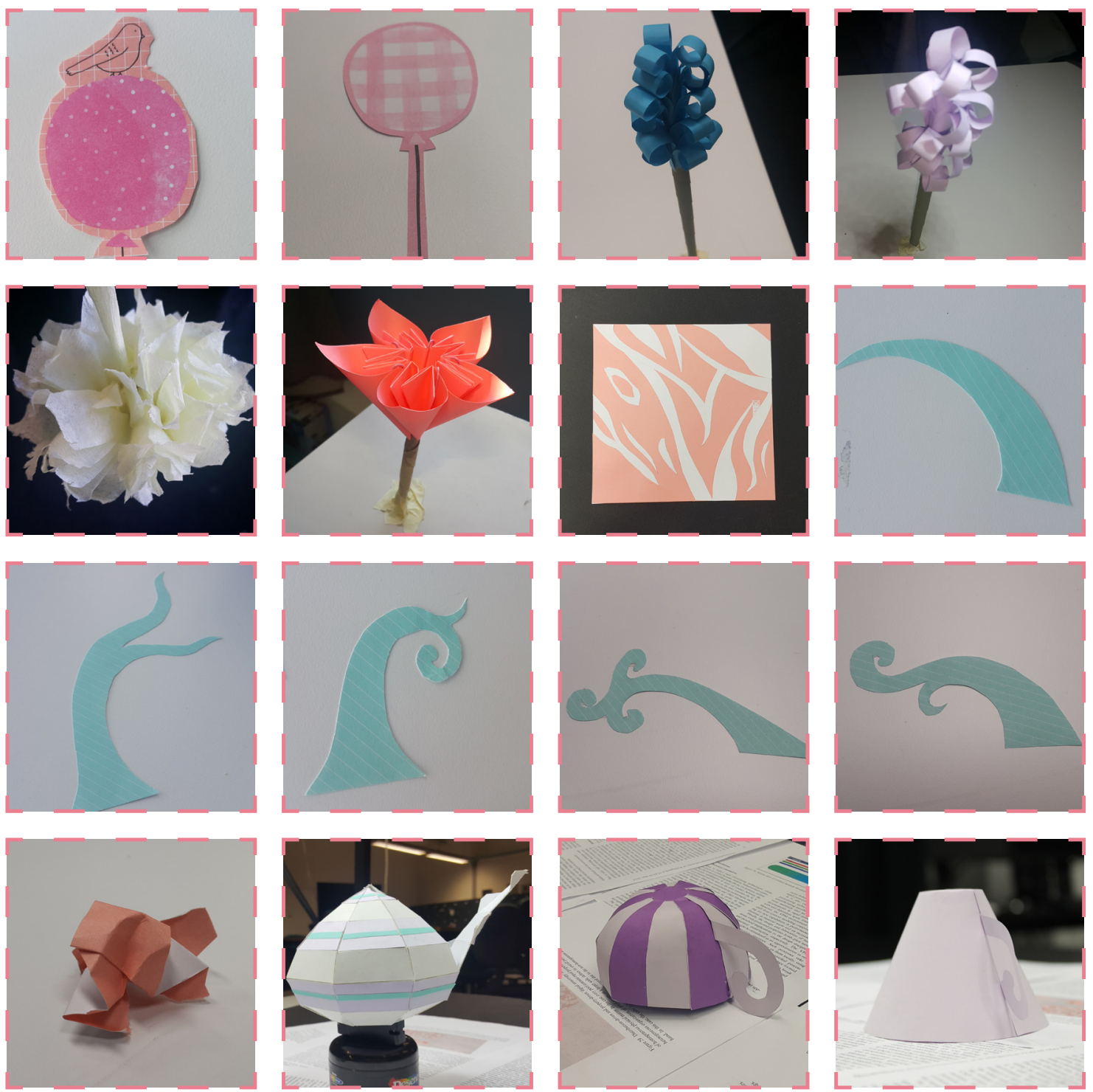

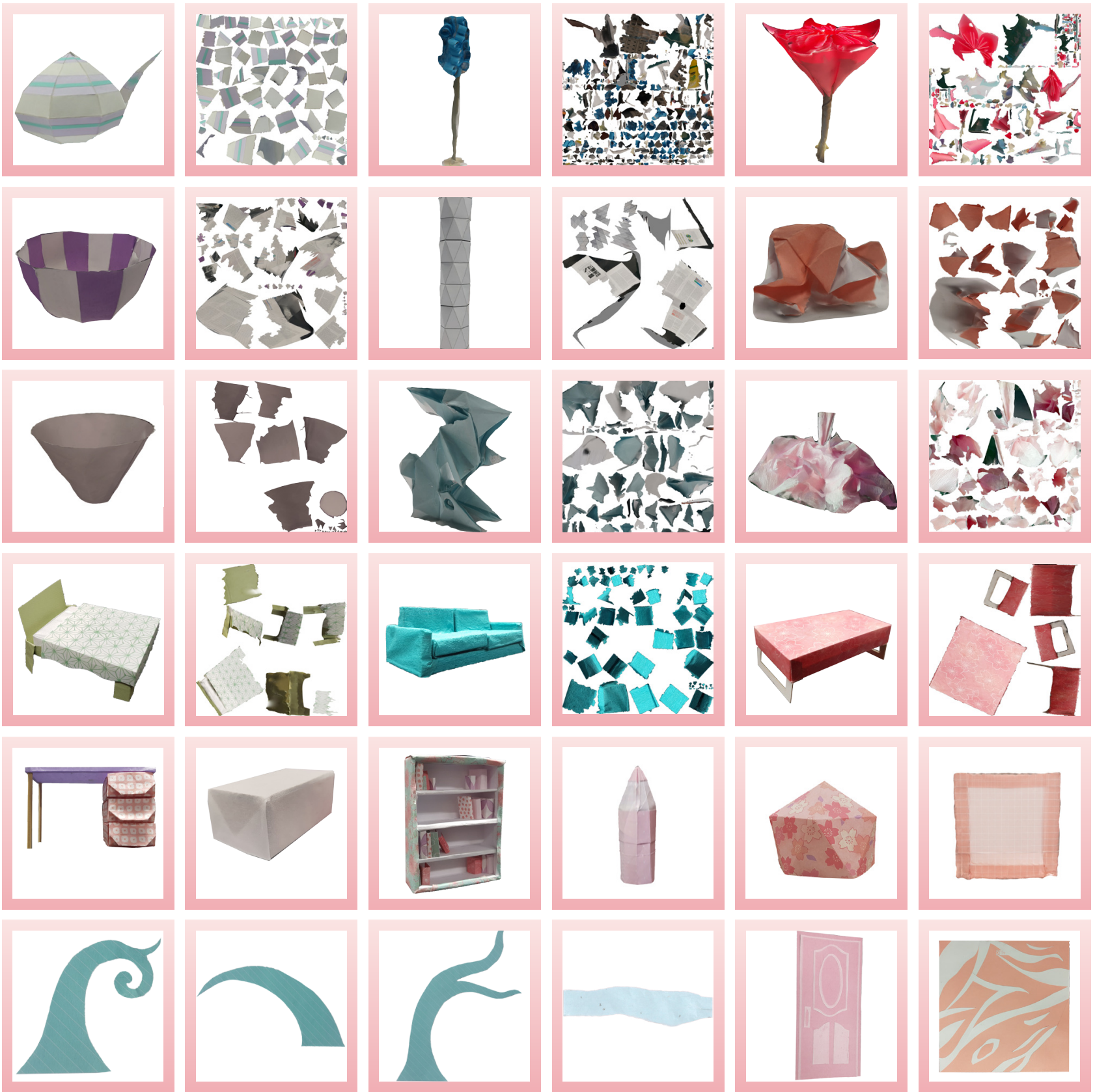

Fig.34. Photogrammetry Model (+ Texture) 

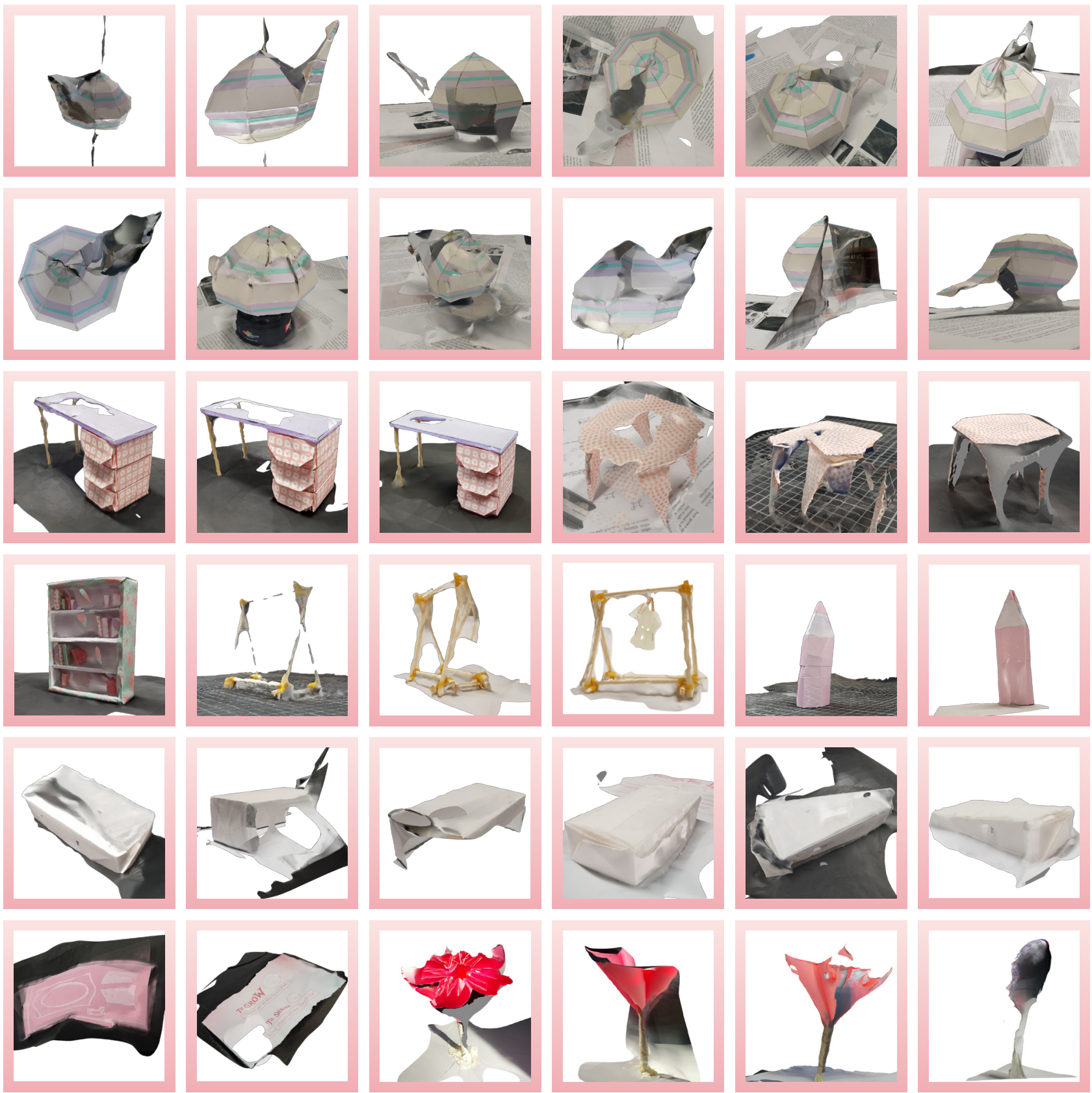

Fig.35. Failed Photogrammetry Model 


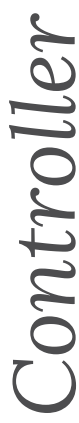

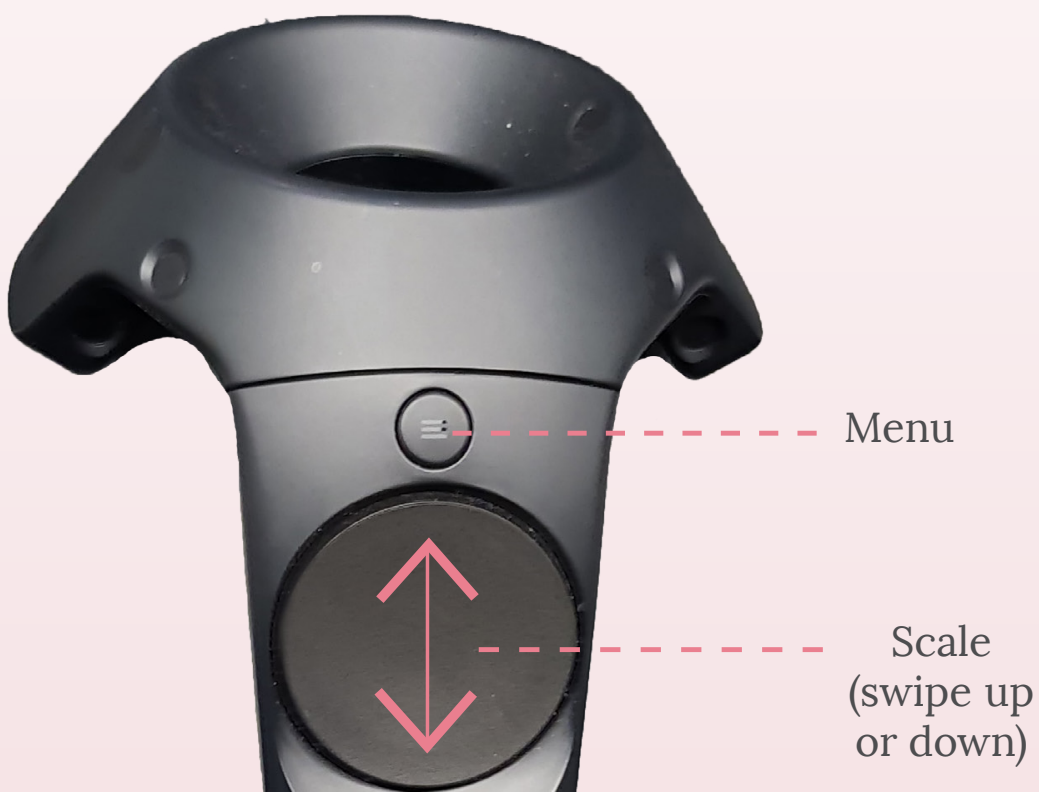

Fig.36. VR Controller Instructions 


$$
f
$$


For this scene I have decided to create the entire environment out of digital polygons and use digital textures. This was done as an introduction to the entire design experience to show the contrast between digital and physical materials. The scene consists of a basic layout of a room with no windows or doors. It is made up of a bed, bookshelf, desk, clothing rack, couches, coffee table and a lamp. The idea of starting in a room is to show the shift in scale as this placed the player in an intimate scale exploring a space with limited accessibility. The players are free to explore the environment but useful cues are added to guide them in the direction which will lead into the next scene (see appendix for code).

The scene is first sketched out with various iterations before settling on a final plan which was then modelled on sketchup. The sketchup model is then exported individually with separate components so they can be modified when imported into unity. The room model is exported with no material properties included as that will be added in a later process. The model is imported and scaled right to be sure it will be a realistic 1:1 scale when experience with VR. A white digital texture is added to keep in line with the paper aesthetic and make the entire design project coherent. Basic lighting have been added to brighten the room. Visual cues of glowing light/flares are added to indicate to players where to go next in order to proceed to the next stage.

Although the scene itself is quite intimate, the 'perfect' nature of digital models are apparent. The digital paper texture used to map over the models did not hide the polished and symmetrical nature of digital models in virtual space. 
+4 Particle System
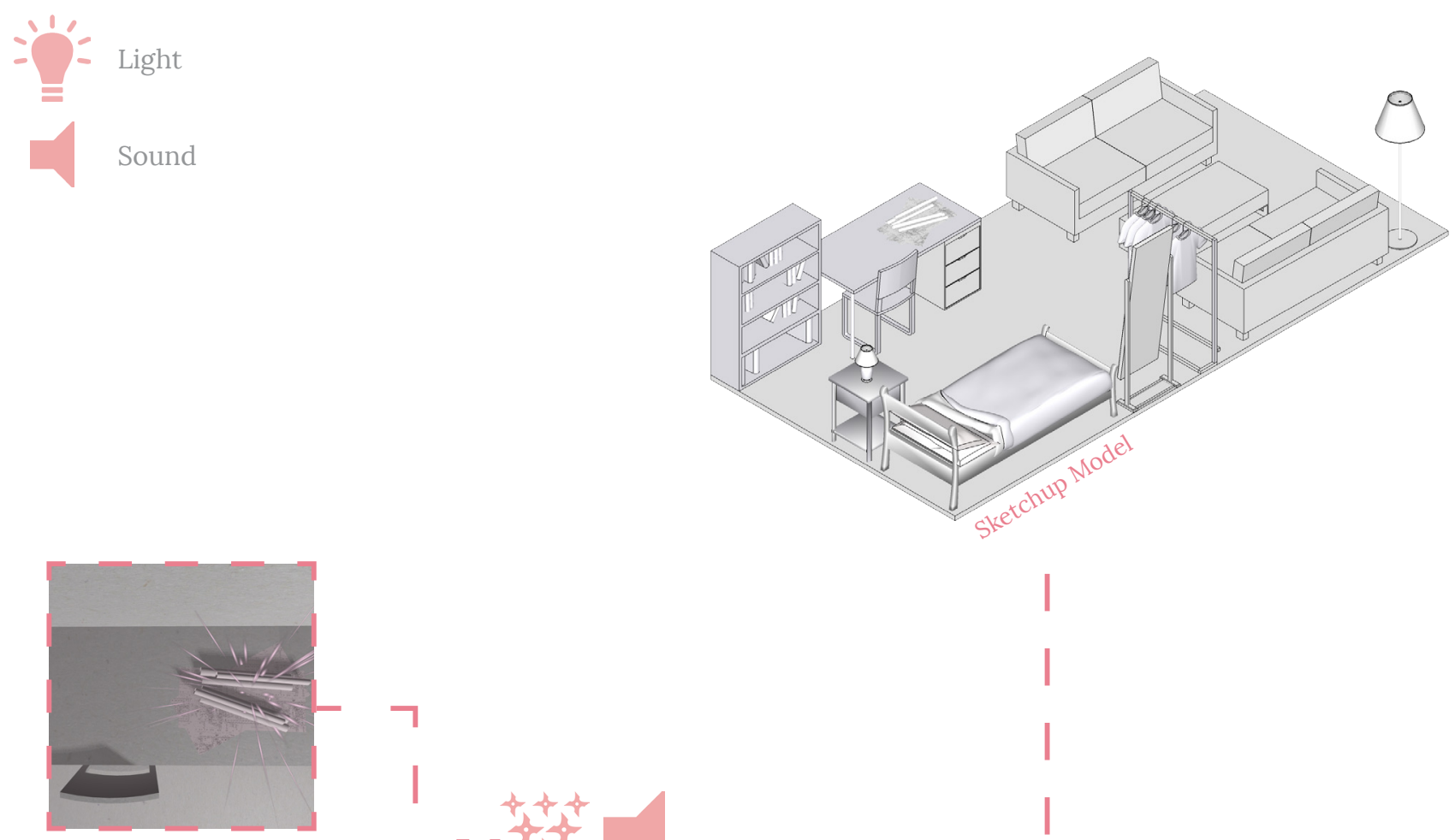

Trigger to enter into next scene

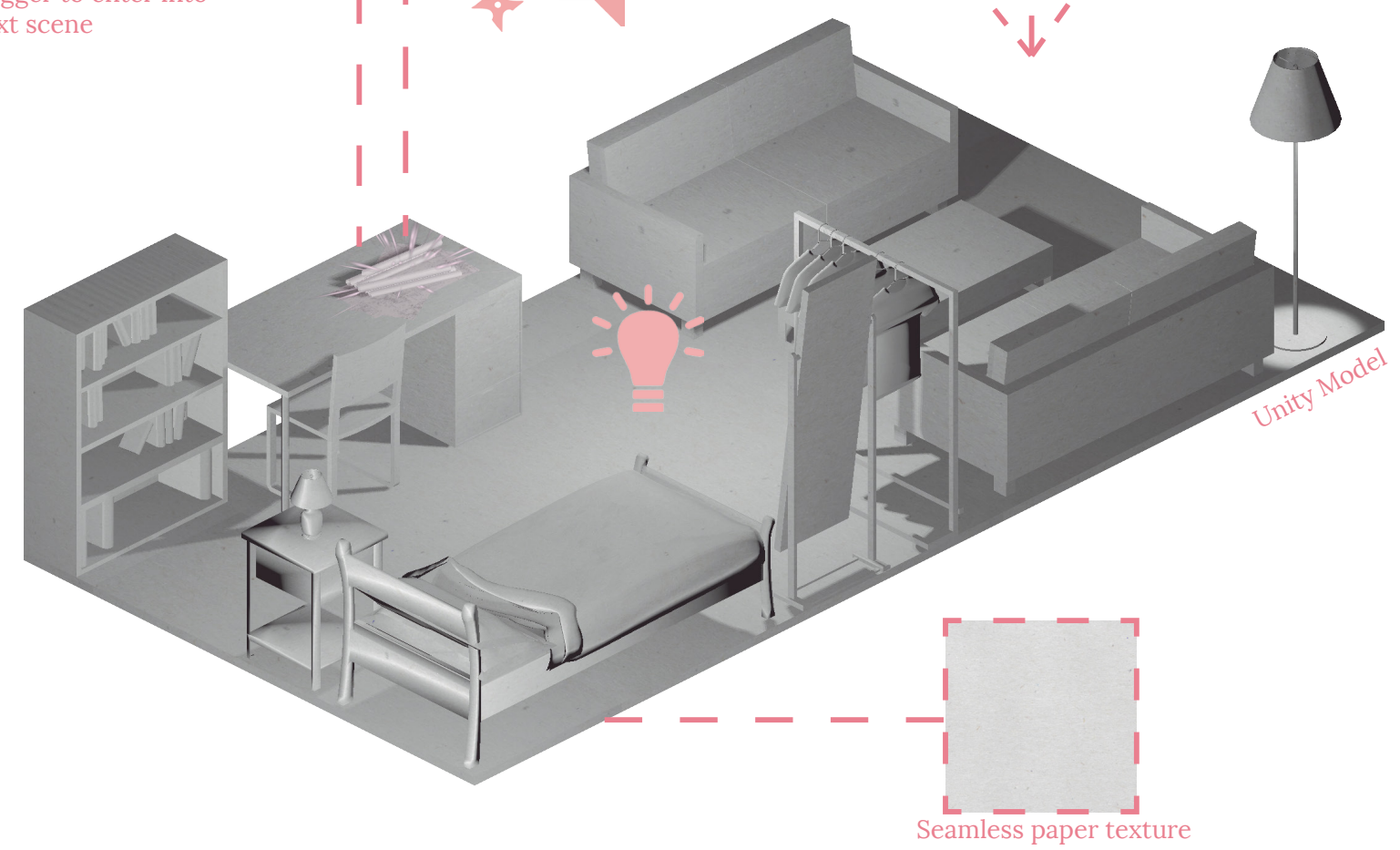

Fig.37. Axonometric view of Scene 1 and the various elements incorporated in order to create an immersive design 

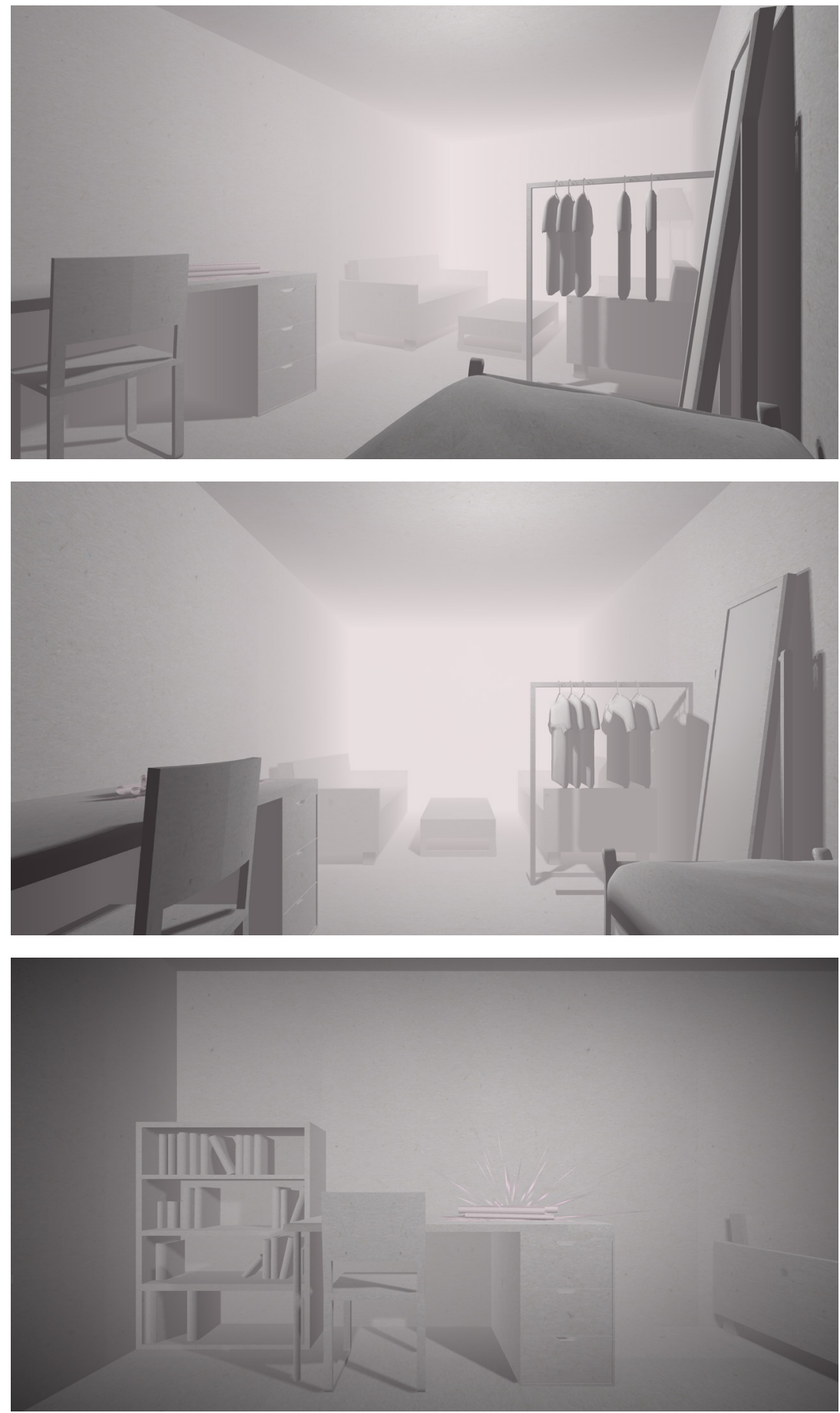

Fig.38. Top: View of the environment from the bed

Fig.39. Middle: View of the environment at the edge of the room

Fig.40. Bottom: View of the desk and and bookshelf with the pink sparks illuminating the plans on the desk. 

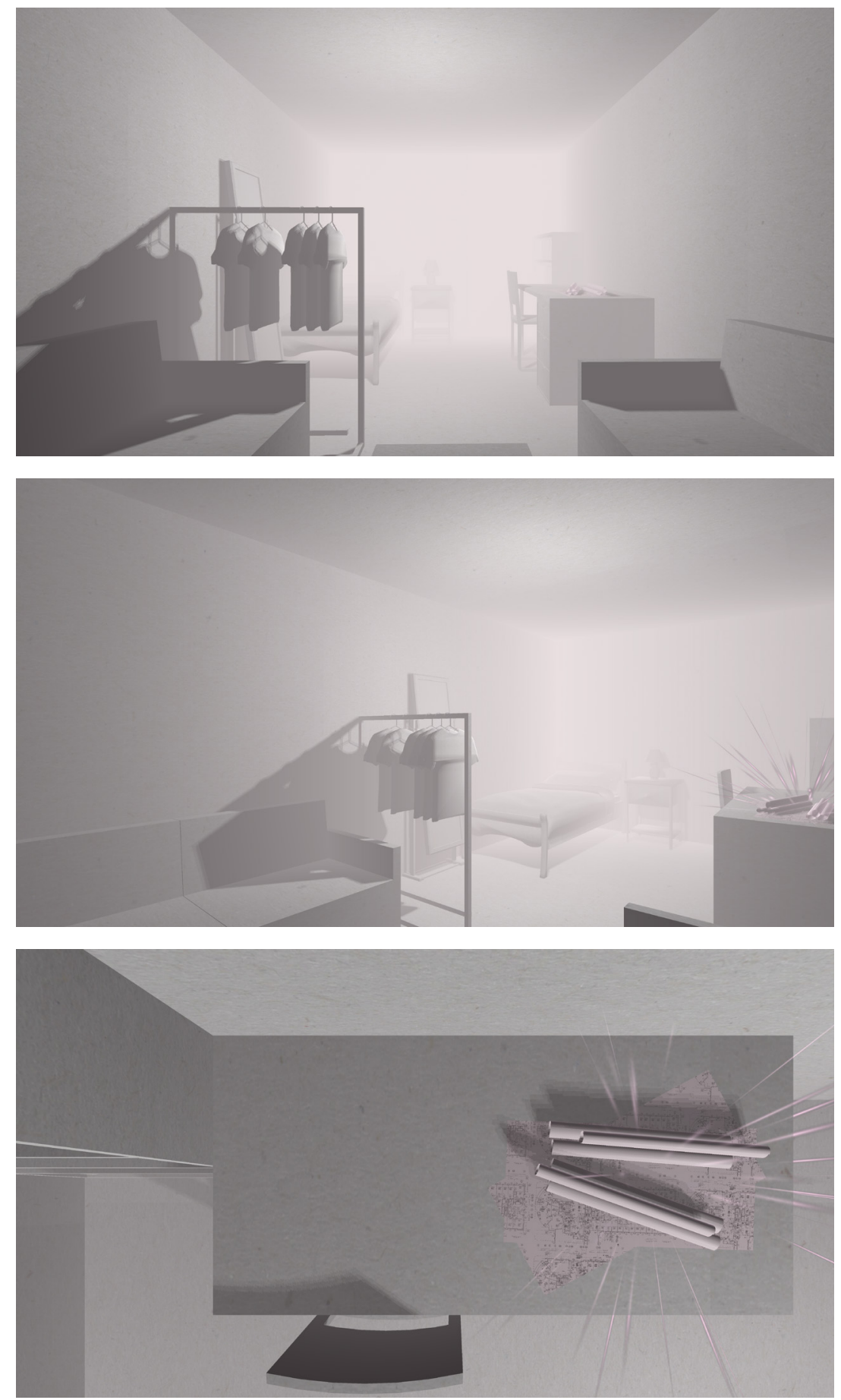

Fig.41. Top: View of the environment from the other end of the room

Fig.42. Middle: View of the environment from a corner of the room

Fig.43. Bottom: View of the desk and the pink plans drawing the user towards the object. When they reach a close proximity to the object a trigger is set off, teleporting them into the next scene 
This scene is used to transition between the digital model towards a handcrafted aesthetic. All the elements are handcrafted and make out of paper. This was a small paper vase that has been duplicated, mirrored and modified so they appear as one continuous rabbit hole. The model was imported using photogrammetry. Coloured lights were added in order to add visual interest and to increase the absurdity of the environment. This scene induce a sense of vertigo for participants as the player experience a sense of falling down. This is made possible due to the immersive quality of VR and the code of decreasing gravity (see appendix) which mimics how we fall in real life. Animation and particle systems are used to further enhance the experience of falling. The rabbit hole is animated to rotate clockwise and the particles systems are speeding towards the player while they are falling, further inciting the vertigo effect. A bright gathering of light sits at the bottom of the rabbit hole which they player falls through in order to enter the next scene. 
to Particle System Sound

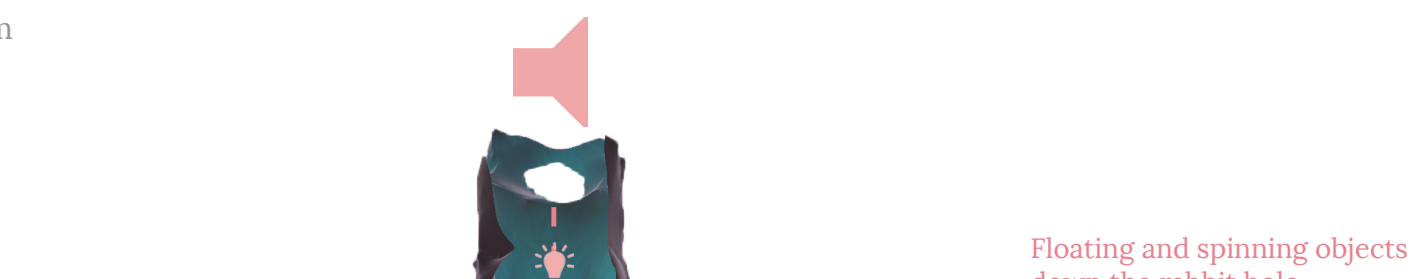
down the rabbit $-\quad-$ hole

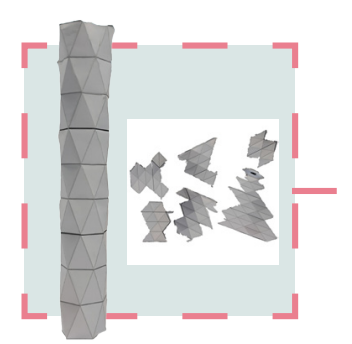

Physical Model + Texture
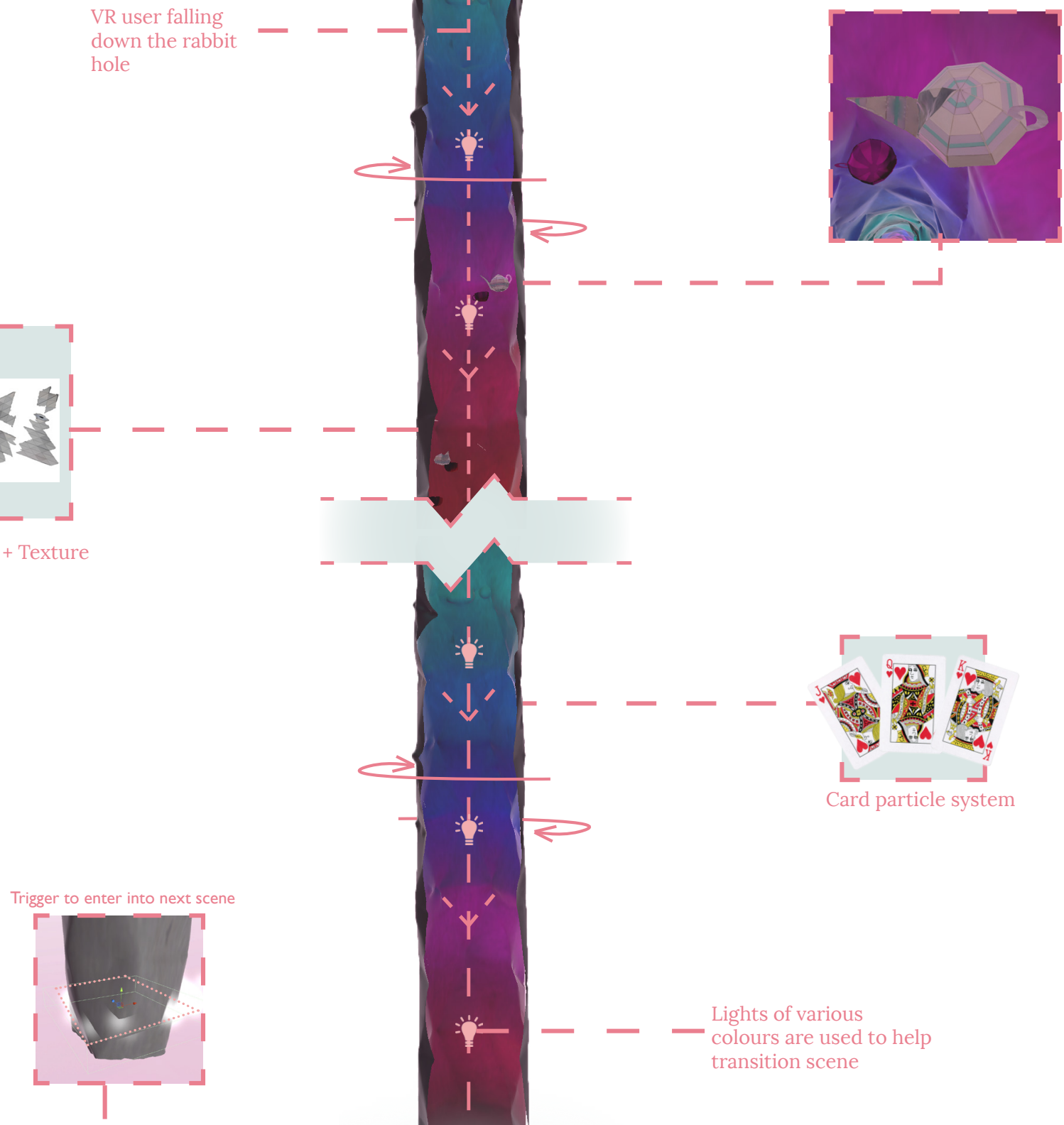

$\underline{L}----\frac{1}{2}$

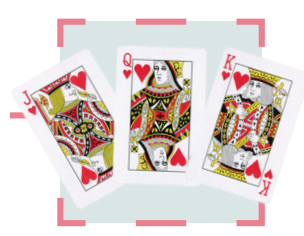

Card particle system

婪

Fig.44. Section view of Scene 2 'Rabbit Hole' 

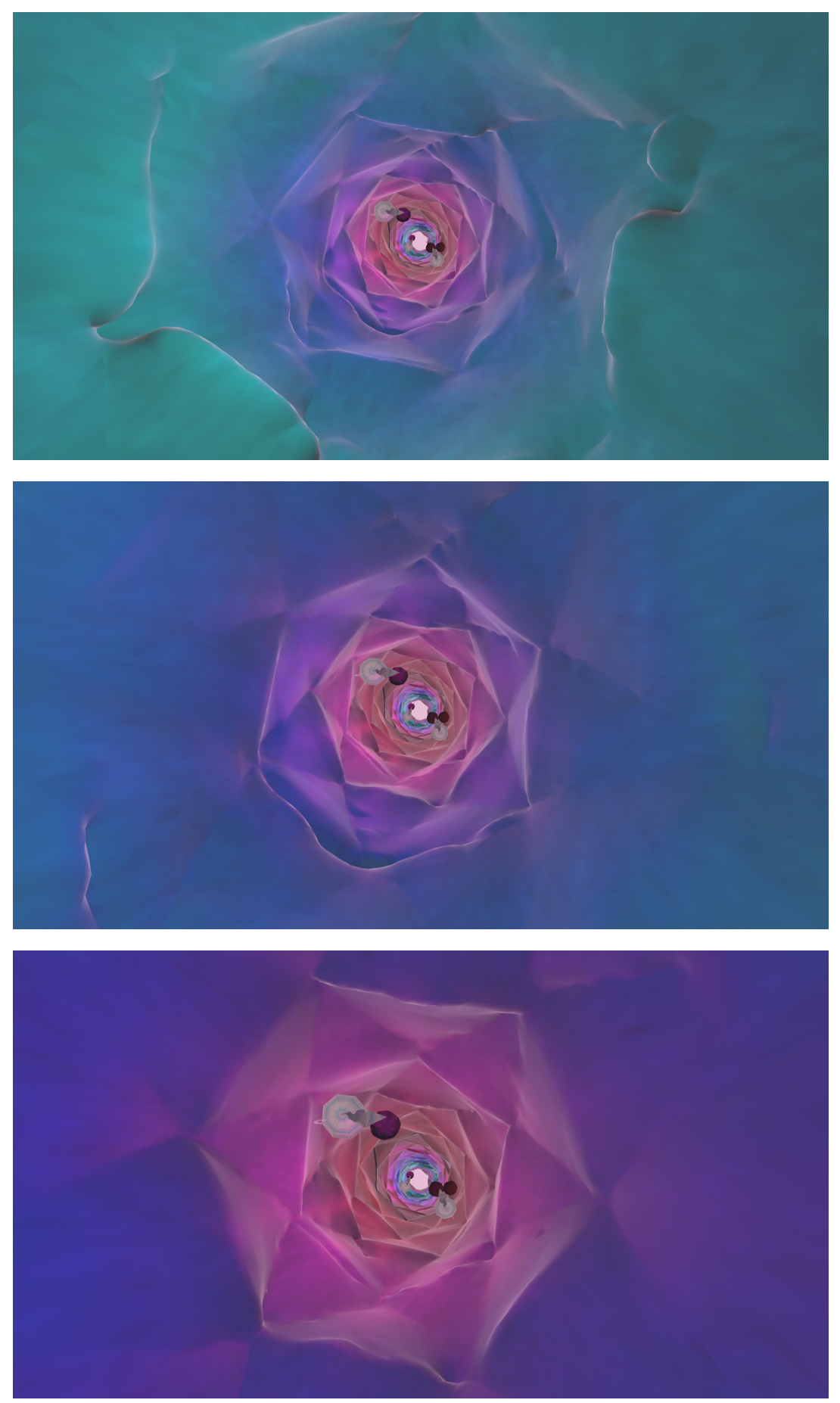

Fig.45. Top: View falling down the rabbit hole (in VR this induce a sense of vertigo) Fig.46. Middle: View falling down the rabbit hole

Fig.47. Bottom: View falling down the rabbit hole transitioning through colours 

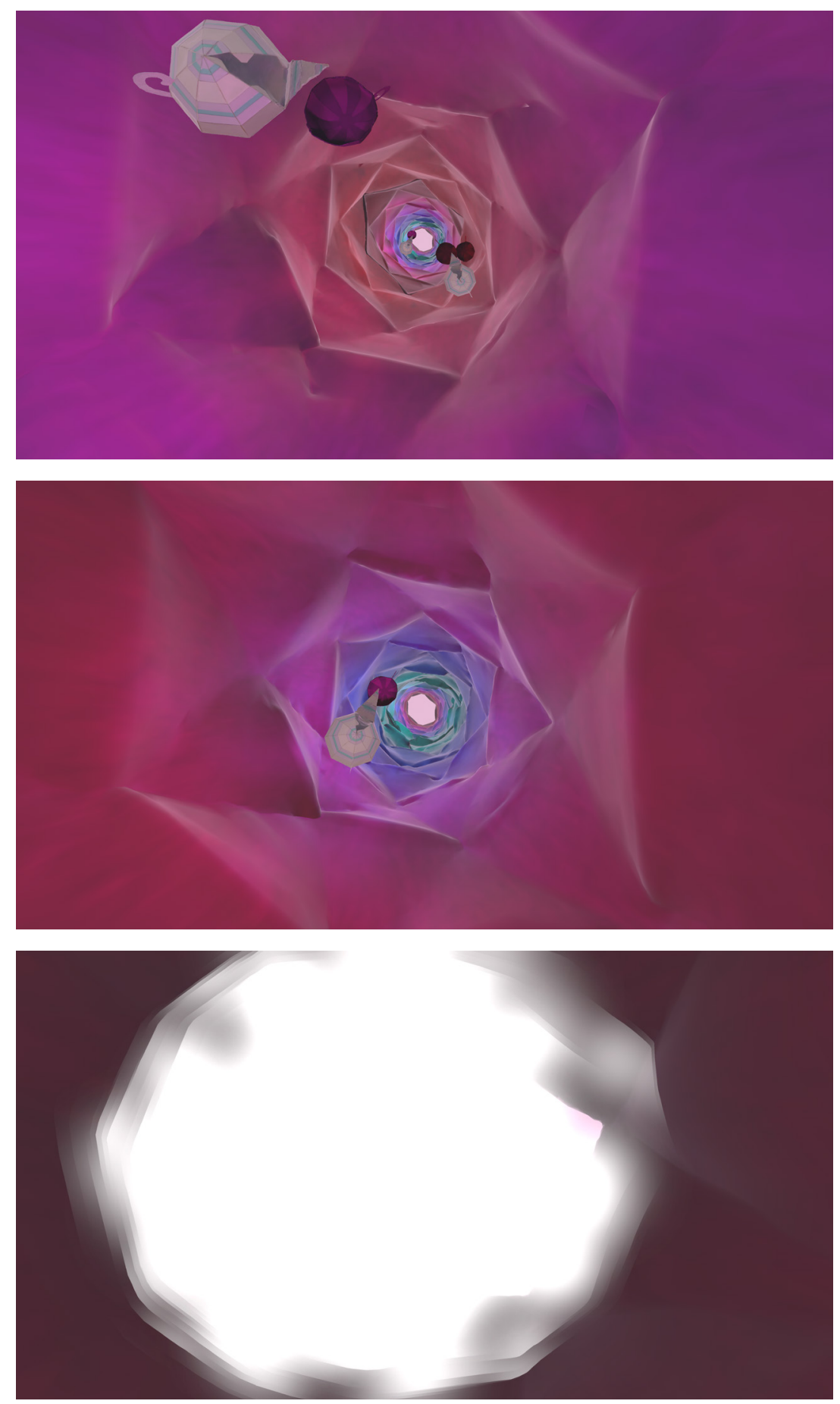

Fig.48. Top: View falling down the rabbit hole

Fig.49. Middle: View falling down the rabbit hole

Fig.50. Bottom: The bright light at the end of the tunnel leading into the next scene 
The next scene is an environment inside a teapot. The curved nature of the teapot provides a unique enclosure full of possibilities. The process of how the teapot was created and imported into Unity is shown above (fig.32). The separate elements used in this scene is the teapot, the hand made door, playing cards, table and note. This scene introduced scale (the ability to grow and shrink) into the design and play with the levels in which they can be incorporated into the virtual environment.

First, the player lands in the middle of the teapot next to a desk with a note. Looking around, there is a clear path way of floating cards leading to a doorway. However, with no way of teleporting to the closest card the only clue is the note. The note introduces the idea of scale and the ability to grow enough to be able to reach the playing cards. This conceptualisation of scale is a subtle callback to the famous scene of Alice drinking a concoction and shrinking in size. By increasing in scale the player can easily teleport onto the playing cards leading up into the doorway which is an entrance way into the next scene.

This scene was created by importing a physical model of a teapot and changing the scale in order for the player to inhabit. The playing cards are physical cards that have been scanned and translated as a texture in Unity. This was then added onto quads in order for the player to interact with the object in the program. Falling cards were then added to the scene as a call-back to the cards that appeared in the previous scene. A code (see appendix) was written for the scale factor whereby if you scroll up on the touchpad of the VR controller, you will grow and if you scroll down, you will shrink. This along with the features already available in the VR controller added an extra dynamic feature in the movement factor of the design as a whole. 
Trigger to enter into next scene

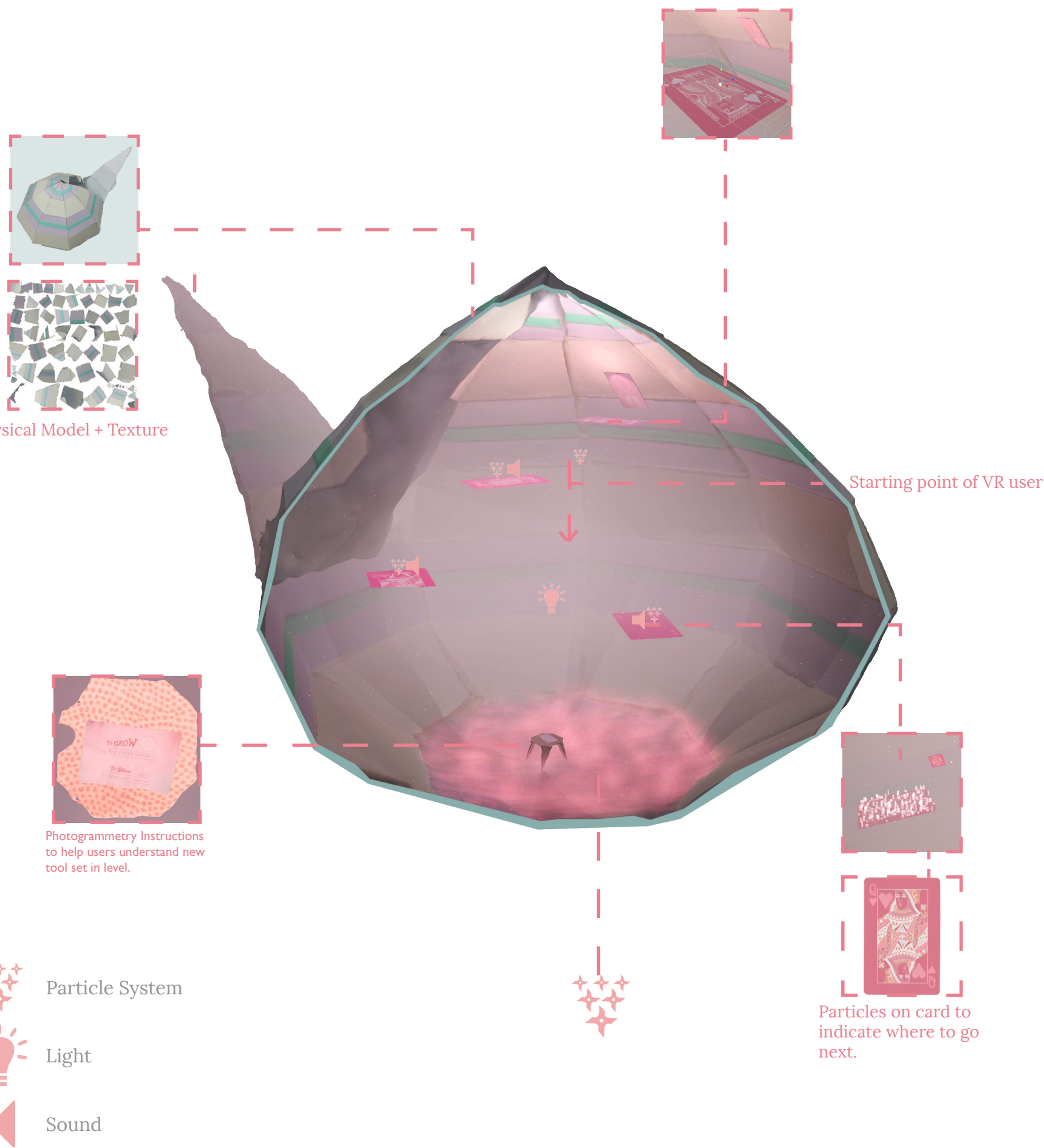

Fig.51. Section view of Scene 3 

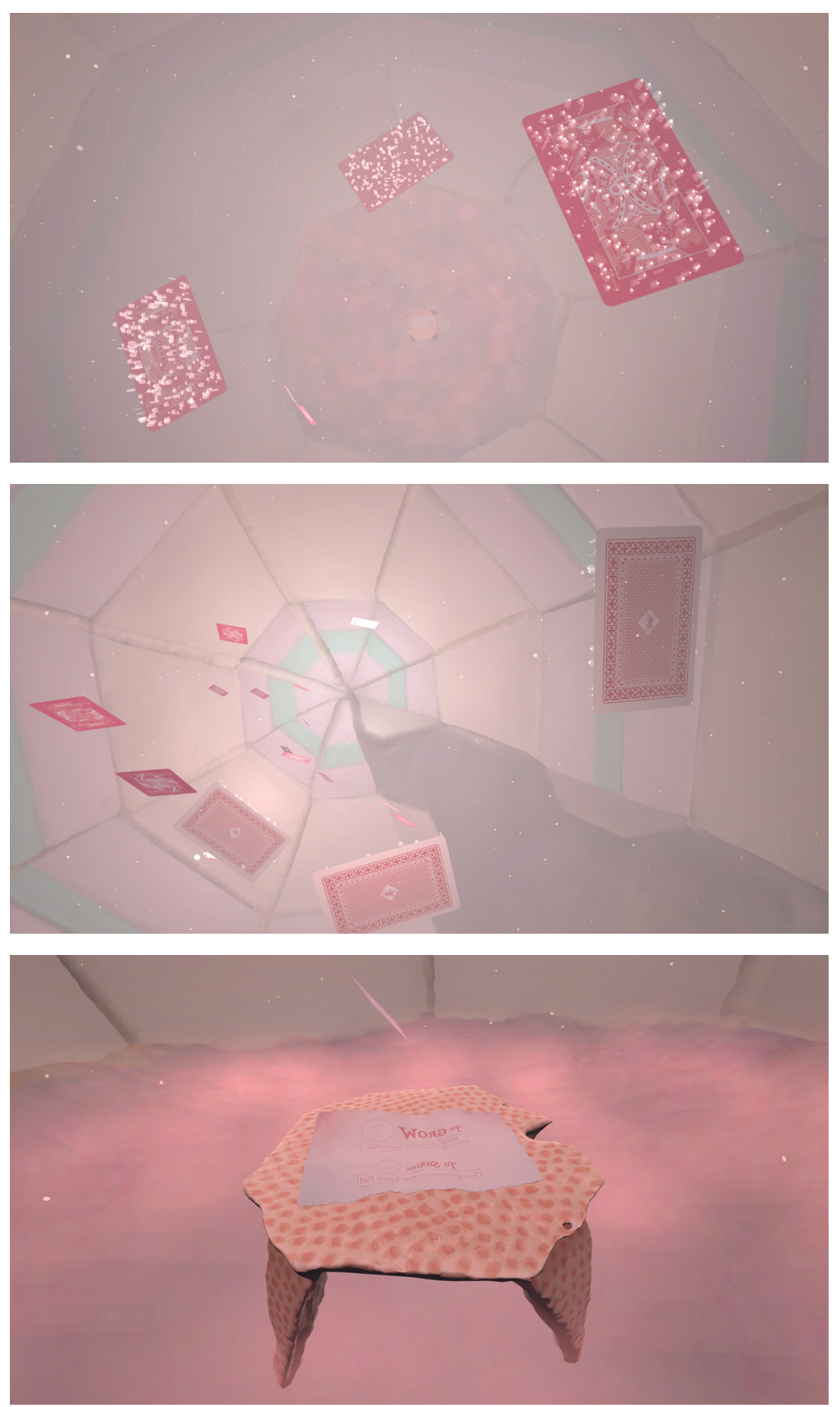

Fig.52. Top: Starting position - view falling down the teapot

Fig.53. Middle: View looking up at the teapot once landed on the ground

Fig.54. Bottom: View of a table with an instruction on how to get grow and shrink 

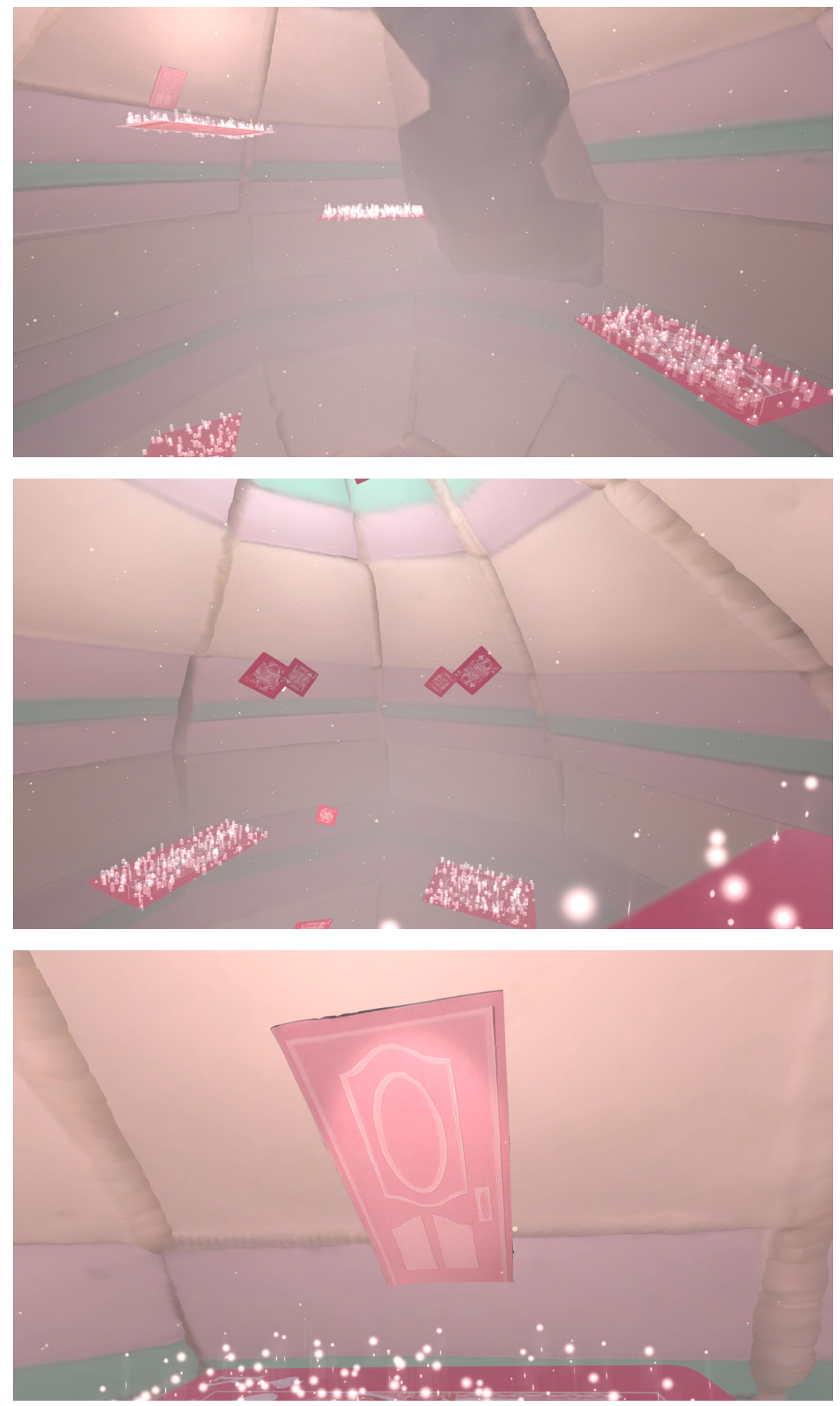

Fig.55. Top: The user growing tall enough to teleport onto one of the card platform Fig.56. Middle: View from one of the card platform

Fig.57. Bottom: View of the door near the top of the teapot leading to the next scene 
This scene is a refined development from the forest scene of the preliminary design. There are vast improvements made as I have reflected on the lack in direction my forest environment previously had. A 'landscape' was created from ripping up pieces of paper and placed together through trial and error (similar to the process of making sketch models). These pieces are roughly placed together which effectively created interesting pathways and focused areas due to the contrasting colours of the paper. This new landscape aid in tightening the area in which the player can explore the environment.

The forest scene is created using the various origami flowers placed in different scale and colours. The balloons are added to increase the contrast of the space, this was done as the two dimensional photogrammetry of the balloons add a visual break towards the three dimensionality of the flowers. These features are flat pieces of paper that have been photographed and transferred with photogrammetry in order to create a sense of depth. Animations have been added to them to bring the forest to life. The animation of the 'balloons' are created by using photogrammetry of the paper art. This was then processed through Remake and cleaned up and modified through 3DS Max. The mesh was then split into the moving head and the stem. Each component was imported separately into Unity and by using the animation toolbox integrated into the program, the animation is formed and played on loop.

The water pond and water area were created entirely by ripped pieces of paper. The paper was scanned and the colours were modified through photoshop. These were then added to the Unity landscape/foliage tool in order for the creator to 'paint' the 2D element where they desired. With this effect, it allows the water element to act as a billboard so the front side is constantly facing the viewer. This technique mimic the movement of water making the pond appear as if water simulation from the unity program has been added.

An origami rabbit is introduced in this scene. Due to the time constraints of the physical presentation, a rabbit was added as a visual guide leading the 'player' around the forest environment. The forest itself is vast and can be explored on its own for hours. This rabbit is coded to move in a certain direction when a trigger box has been breached (see appendix). The rabbit leads the player around the environment in a simple but effective manner, taking in all the unique characteristic of the virtual world.

Post processing elements such as depth of field (DOF), motion blue, fog, particle systems etc are added in order to enliven the scene. Music and audio folly has been added to create a sense of space.

The ability to change the scale of the player is still available although it isn't necessary to explore the environment. 


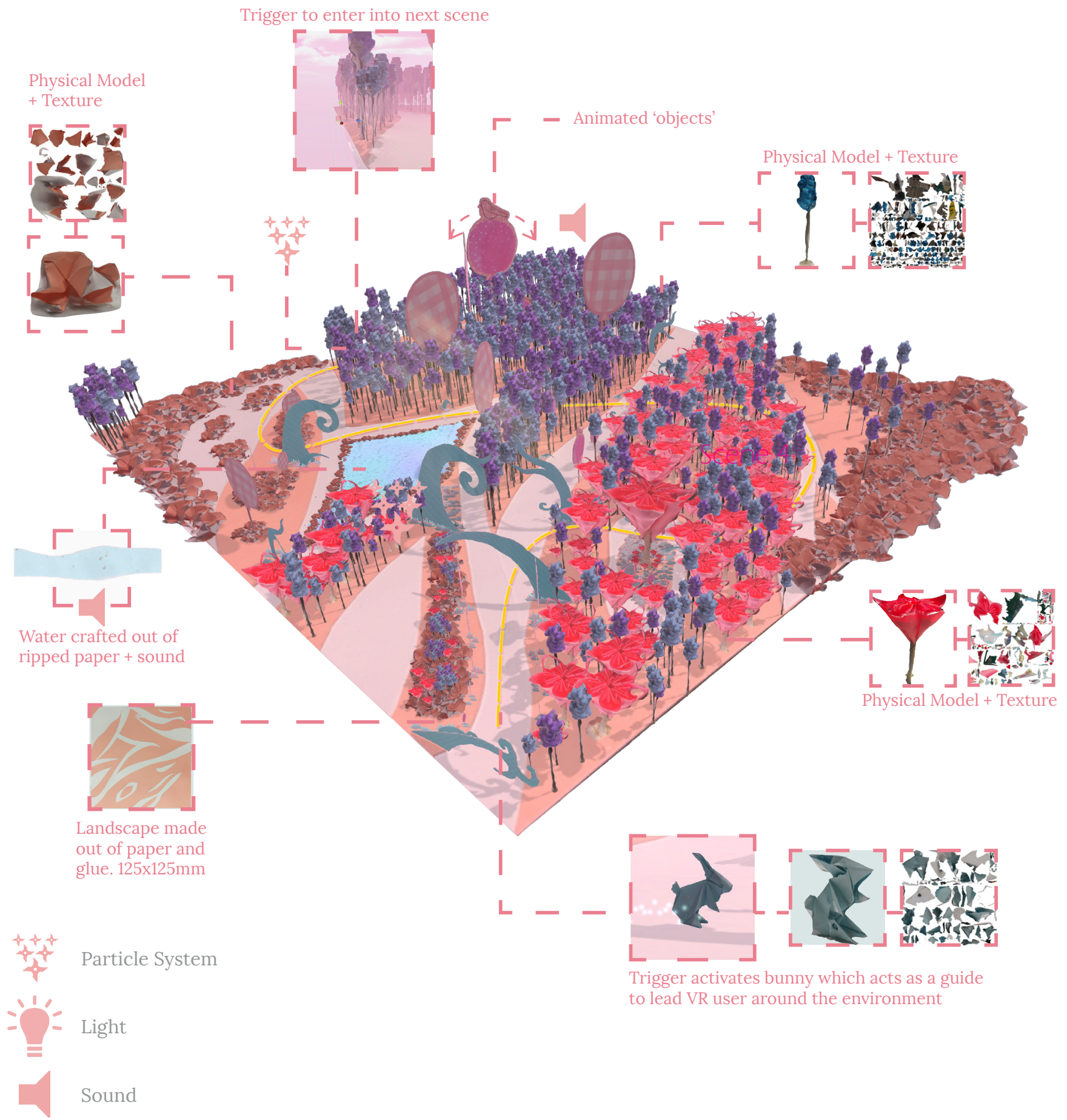

Fig.58. Axonometric view of Scene 4. The yellow line indicates the pathway the bunny takes 

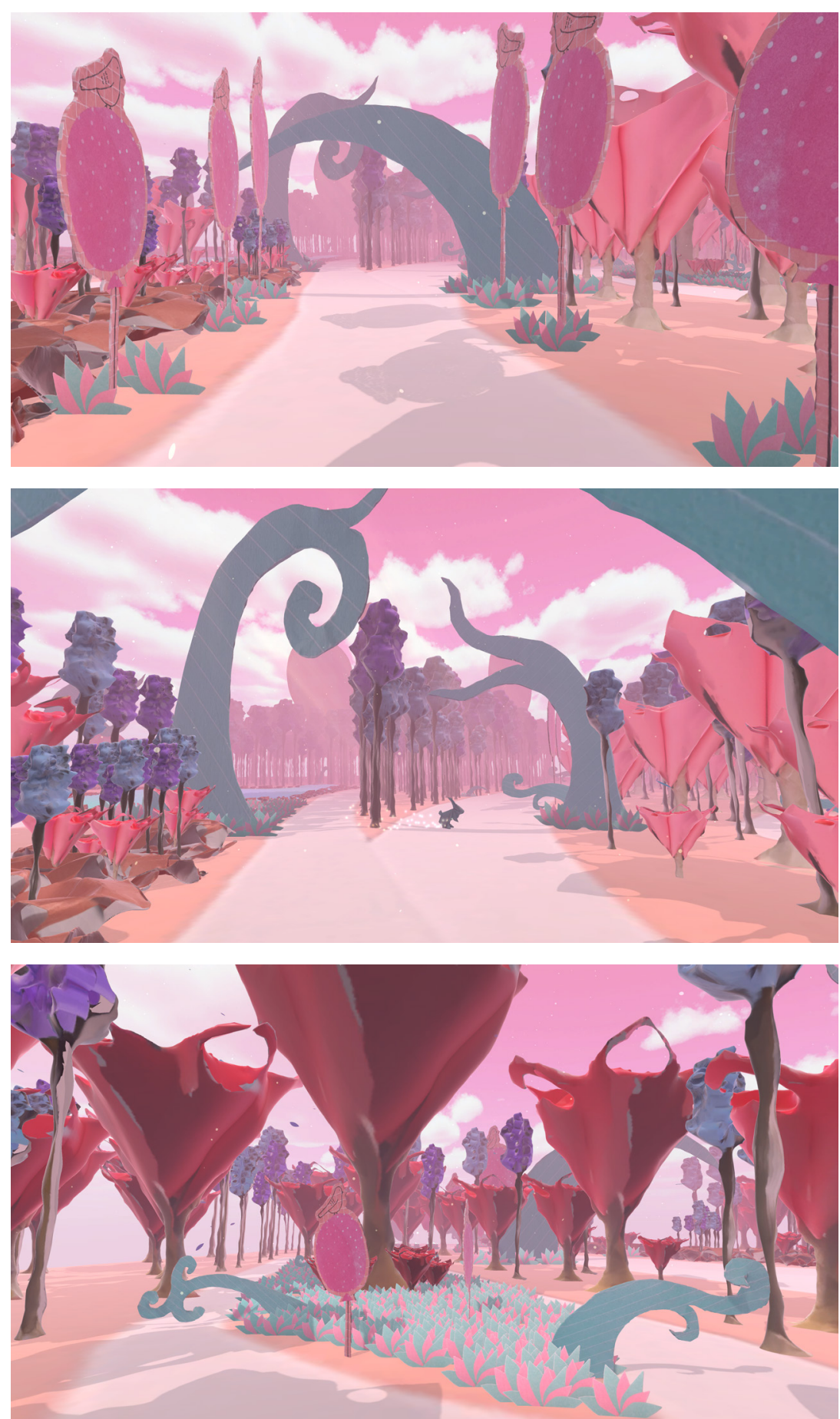

Fig.59. Top: Starting position of scene 4

Fig.60. Middle: View of the rabbit across the screen prompting the user to follow

Fig.61. Bottom: View of a mini garden area composing of elements from the preliminary design stage 

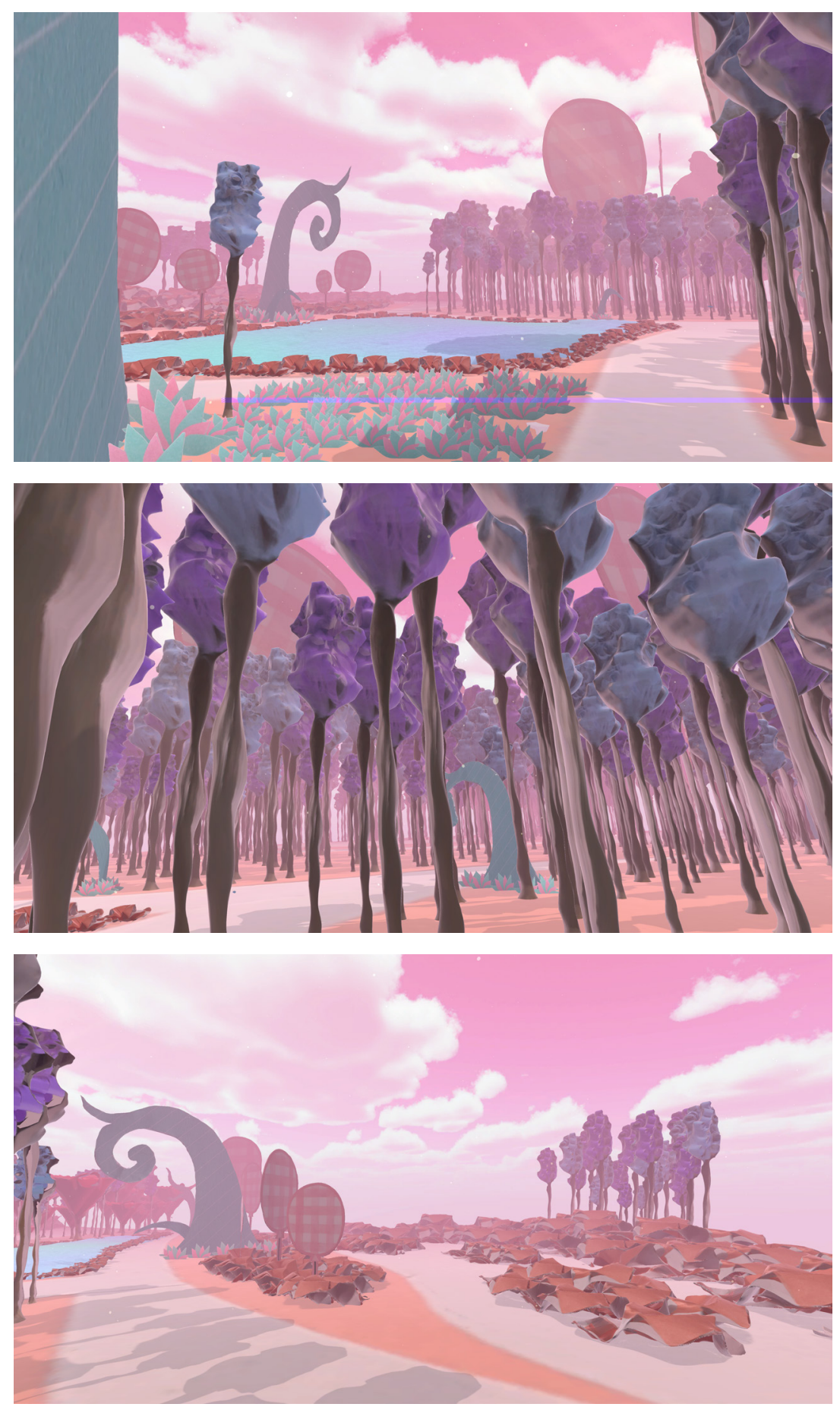

Fig.62. Top: View of the pond made up of ripped paper placed together densely Fig.63. Middle: View from within the forest

Fig.64. Bottom: View of the rock garden 
This last scene is a representation of the first scene but in contrast is entirely made out of paper craft models. It begins with the inhabitant entering the scene onto a desk, where behind them was the forest they have previously explored. Everything around the room is oversize. This is done as a callback to the thesis topic on using scaled models. The player was entering a scale model from the moment the player has 'fallen' down the rabbit hole. Now the player is re-entering the 'real world' and from what the player has learned and experienced in "wonderland" it helped to brighten and see the world in a different perspective. Having the forest scene on the desk as a scaled model provided a tie in with the entire narrative of the design.

As the player explores the space and 'falls' off the desk, the player is once again increasing in size until the size is back to human scale. The player can then explore the room as it is and can compare the contrast between the white digital room with the room full of paper material of different textures.

The idea for this scene is not to be a direct replica but to have enough similar components to feel the same. Certain objects in the scene are elements that have appeared in previous scenes but have been repurposed as something else. This is a way to tie in all other components in the entire narrative design in order to form a coherent world.

Each aspect of this scene is built and made in different scale. This is because of the amount of detail and care different scale provides for various reasons. The desk on one hand is made much larger than the bed. This is due to the fact that the desk has many components adding to the overall function and final outcome, whereas the bed only needed a few fold and cuts in order to stand and function for its use.

A slight background ambience is added to generate noise in an enclosed room. The ambience added extra depth to an otherwise small room. 
Particle System

Sound

Starting point of VR user in small scale
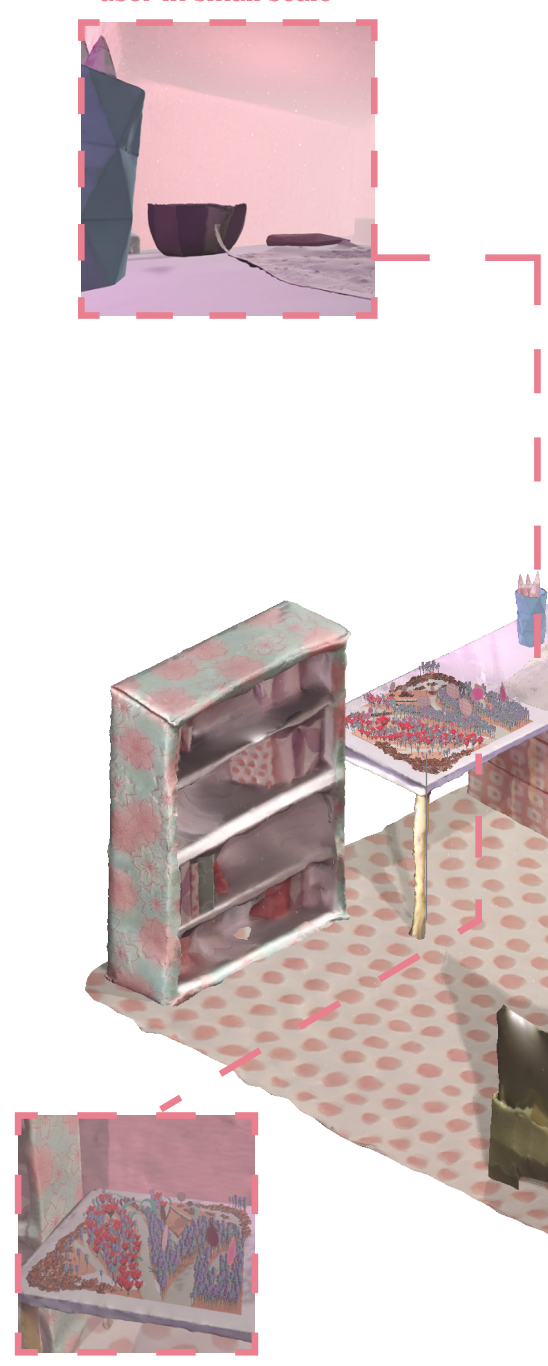

Scaled model of forest scene on desk
Trigger that

turns the user

back to 1:1

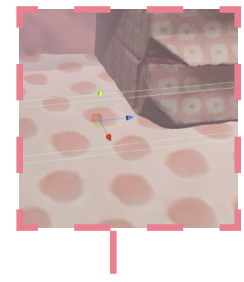

Physical Model + Texture

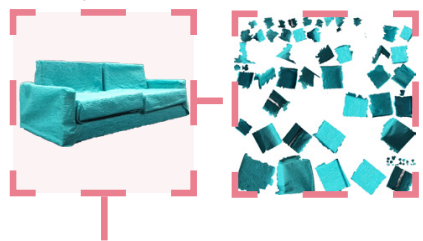



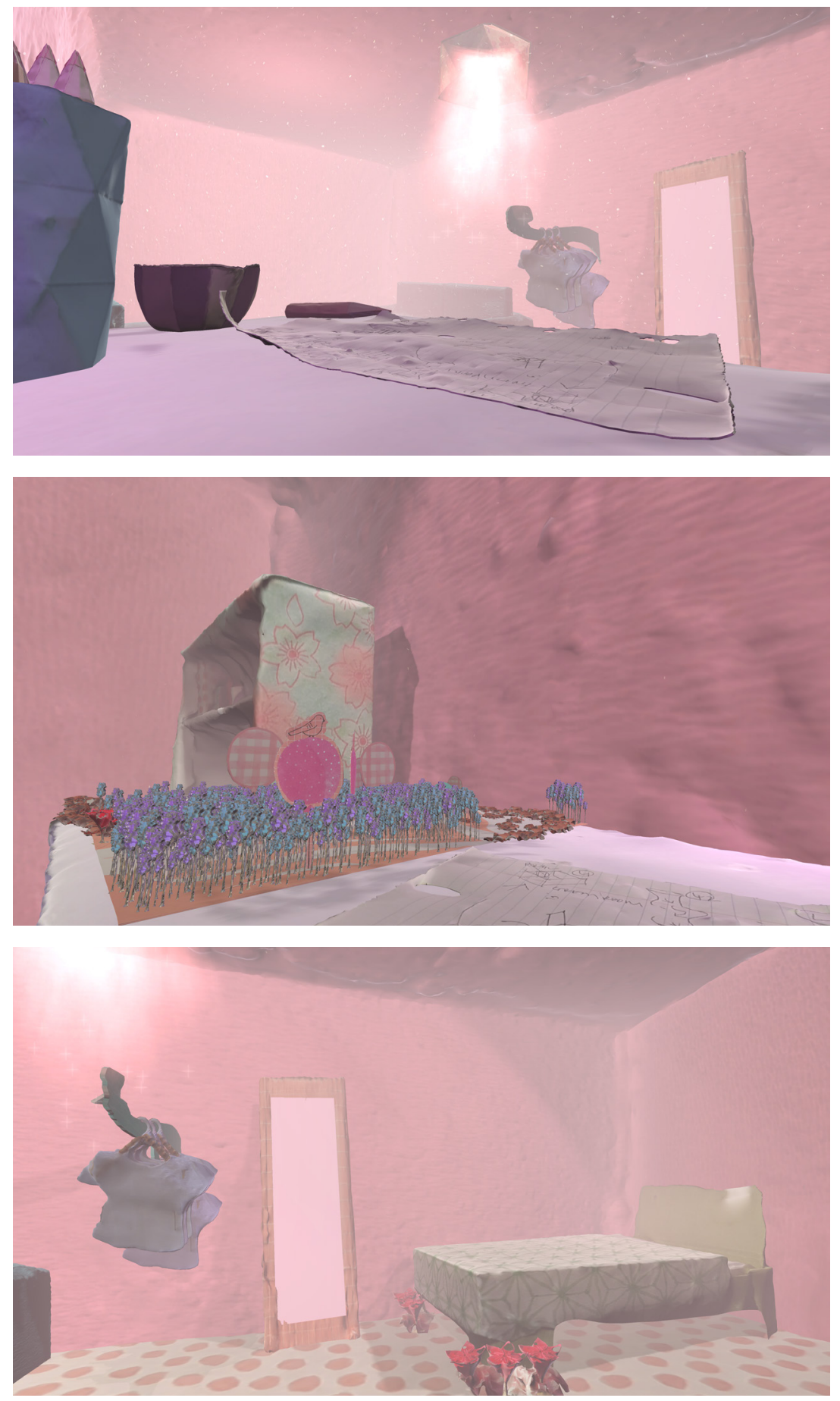

Fig.66. Top: Starting position - on top of a desk

Fig.67. Middle: The scaled model on the desk is the forest from the previous scene Fig.68. Bottom: View of the room from the desk 

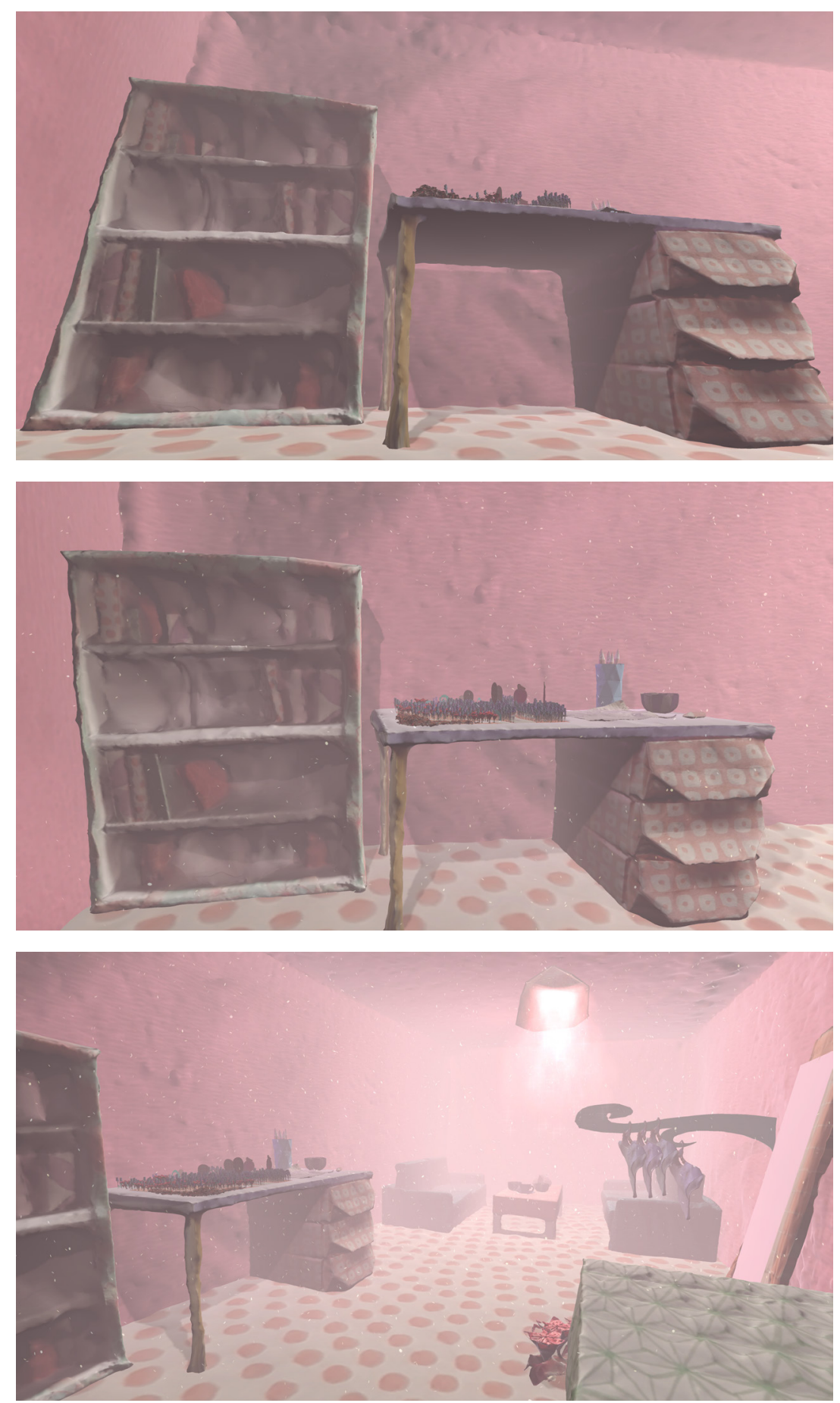

Fig.69. Top: View from the ground (still as a scaled figure)

Fig.70. Middle: The user is growing in size

Fig.71. Bottom: User now at full (human) scale and can explore the environment freely 


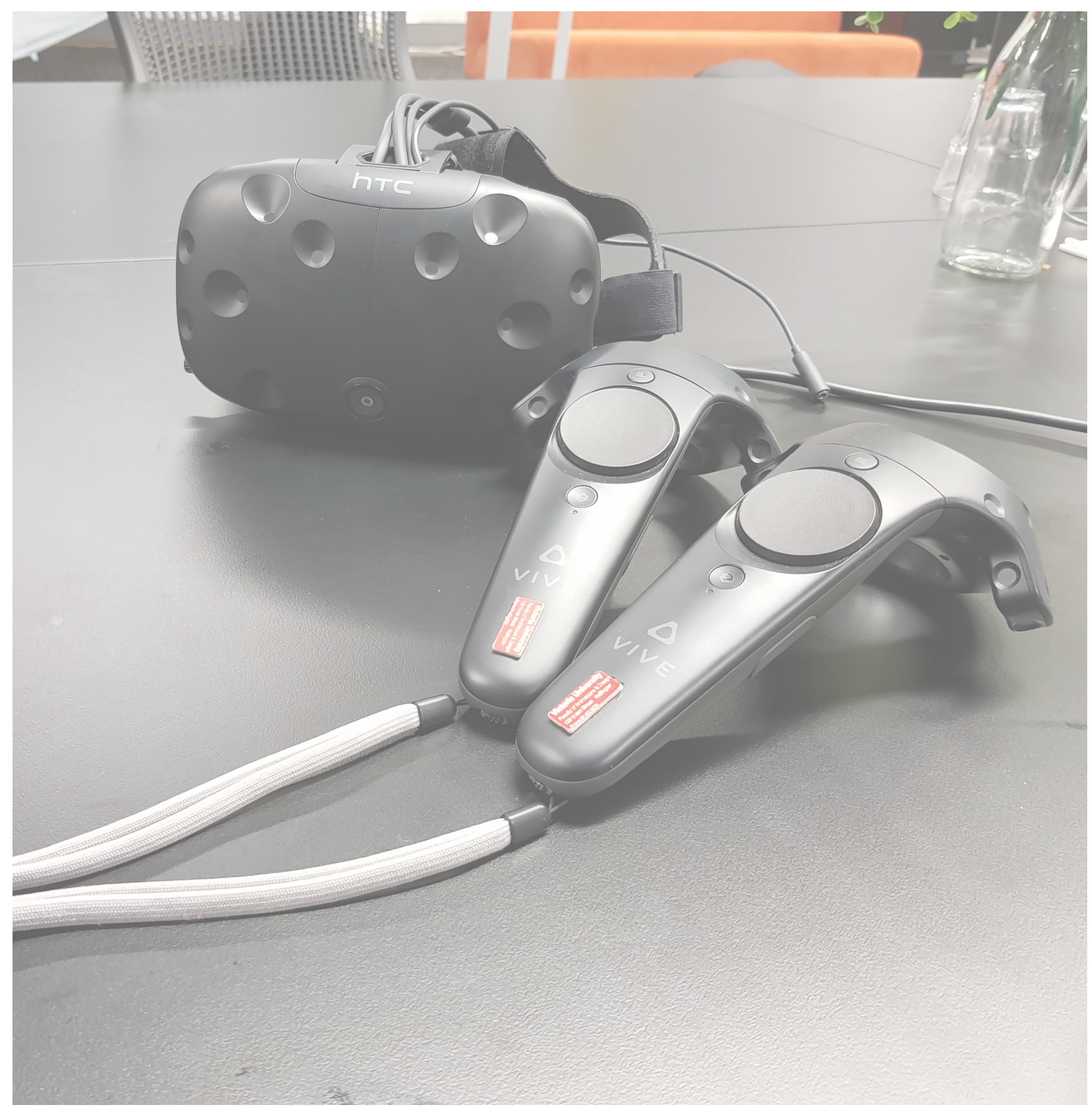

Fig.72. VR system used for the design process 


\section{Chapter 6:}

\section{Conclusion}


The research question for this thesis is:

How can Real-Time Virtual Environments be used to bridge the gap between material properties of the physical world and the perceived lack of materiality of the digital?

This thesis proposal started as a means to understand ways in which the physical world can cooperate with the digital. This proposal is brought about due to a concern that as we are living in a digital-heavy reality with new technologies and tools constantly emerging, the use of traditional methods (in architecture) are being ignored. The research question is answered through thorough exploration of the design process; testing various scaled paper models and using them as a alternative way to content create in a digital space. Materiality of physical models are called into question in relation to digitally created models. It translated the warmth and imperfection of materials in scaled physical models into a digital medium in which people can interact with it in a 1:1 scale, three dimensionally. These translations of material properties are not a method in which to replicate photorealism in the virtual realm, but instead are used to introduce an approach to the abstraction of form and how we can use it to create impactful virtual environments. 
In the Literature Review I discussed the issues of the primary form of representation in the architecture discipline. The use of static images in the conceptual and final phase of a design has limitations as it only illustrate what is presented and flattens the reality of what has been designed. Physical models is another form of representation common in the discipline, but the scale factor, although useful in examining the overall context of the design, limits people's understanding of the physical space and the intricacy of the craftsmanship is lost.

Through these theoretical context studied, it has helped me to find my position and direction for this thesis. Venturing into ways we can bring the use of three dimensional exploration into the design phase, I have decided to use virtual reality as a means of exploration and showcasing my final design.

The Case Studies helped me looked at various examples of work outside the architecture discipline and how physical models can be used not just as a representation method, but as an environment for people to explore. These examples successfully showcase how architecture can be used in other mediums, exhibiting ways in which people can gain spatial awareness and agency without physically being in the space. These case studies were enlightening as they helped expand my creative thinking and illustrate how architecture can be used and represented in more than just the conventional methods.

As I did not have a site to work with, I had chosen to use a well known narrative in order to guide my design. From a range of stories, Alice in Wonderland was chosen. Scale was a major factor that linked the story ideals with my design intent. The ambiguity and fantastical aspects of the story fitted well with my chosen aesthetic and allowed the freedom to create interesting environments that deals with scale, materiality and the imagination. 
The preliminary design covers the exploration I did leading up to the three month review. In this stage of my thesis, my outcome and direction has not been finalised. The act of designing in order to gain knowledge and narrow my scope was a helpful method in exploring my options to figure out what works. The explorations provided a roadmap for my thesis research. Simple physical objects are created first in order to test methods of using photogrammetry. Successes and failures are documented and the few elements created were used to test out the initial virtual environment created in Unity. This allows me to analyse my process and the method of construction and photography that could improve the efficiency of transferring a physical model into a digital mesh.

The final design took the knowledge I have gained through the preliminary design and improved on the efficiency of the process. I have built a cohesive narrative through five interlinking scenes that tackled the research question through users exploring the virtual environments made out of physical models. This stage delve into the usage of Virtual Reality; coding, animation and visual effects are used in conjunction to expand the experience. This is done as VR is representing more than a static image, which means participant's spatial awareness are taken into consideration when designing for a RTVE (real time virtual environment). The scenes are created out of order due to the environment I have envisioned at the beginning of my research. The forest scene is first as it continues from my experimentation in the preliminary design. However, I am constantly learning and perfecting my techniques throughout the entire design phase so sense of quality in the design is expected.

The mix media usage of incorporating hand crafted models (those from the physical world) and taking it into virtual reality highlight a closer connection between the representation of a design versus what will be built. The design allows participant to use their spatial awareness in order to understand an architectural space during the conceptual stage. Motion, time, memory and sound are successfully used to enhance the experience activating more than the visual sense in representation. This thesis process presents an alternative method to designing with the material and craftsmanship of the real world combined with the easier manipulative methods of digital technologies and programs. 


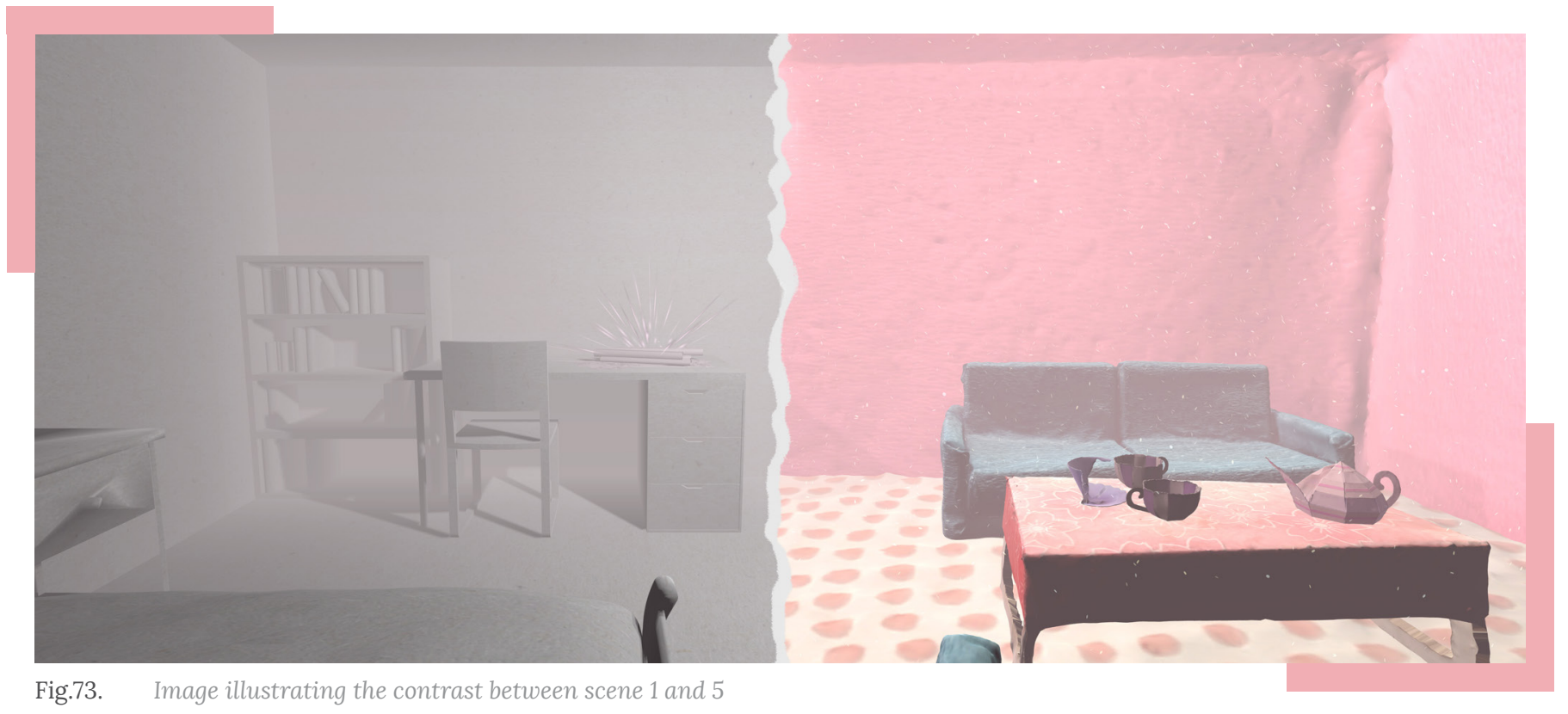


Having never worked with VR before, this thesis research is a rewarding challenge that pushed me out of my comfort zone in order to acquire, test and develop new skills. It is a tremendous learning curve which helped me to look at an alternative way of designing outside of the conventional methods typically used during my education. Physical modeling is an approach I enjoyed using in my design phase so I am happy I could merge both methods of designing together. There are many benefits in doing this as the constant feedback between the digital and physical allowed the advantages of both processes to create a design of high quality.

The material chosen for the focus of my design is paper. There are many different construction materials available but ultimately, paper is the most versatile and easily acquired. It can be used in many different ways and still look delicate which matched the aesthetic I was striving for in my thesis. By modeling physically with paper, it helped me understand the limits and flexibility of the material; from the variety of thicknesses to their abilities to withstand malleability and manipulation needed to form an outcome. This is a great knowledge to learn as it helped me identify which type of paper is ideal for the various models I needed for my own design.

I understand that physical material properties can expand to many different factors (and the use of a variety of materials to get different outcomes). However, I have decided to focus on an exploration using the characteristic of paper as my main material due to its versatility. As I only had a year to conduct this research, it seemed reasonable for me to narrow my scope so there is more focus on the quality and knowledge gained through using a single material. This also kept the language of my design cohesive throughout each exploration. 
Being one person behind the entire design production, there are a few limitations throughout the entire research process. As I am new to VR and designing using a game engine, there are many things I needed to learn in order to make something appear adequate. The limitation to my knowledge on coding and animation is a hindrance to create exactly what I intended. However, these challenges pushed me to think creatively in other ways in order to achieve what I strived for in my thesis. It helped expand my knowledge and design thinking. VR as a design tool also has its limitations as currently there are only a selective amount of VR gears available in university education. The majority of the students do not have access the equipment making it difficult in pushing for this method as a means of new representation in the design studio. I am not suggesting that VR will be a new method to replace the ones we have currently, but as an alternative to design thinking and creating through the conceptual process of architecture.

I was ambitious with what I wanted to achieve at the start of the year but realising the scope in which it takes to create even one scene had me reassessing what I can accomplish in one year. I feel my final design was a success not only as an outcome but from what I have learned throughout the entire process. From this research study several conclusion are drawn:

1. The hybrid medium offers a different method of articulating geometry and offer an alternative approach to designing.

2. Digital and physical materials and models are not separate and there is a clear understanding of the function between them in reality and the virtual environment.

3. RTVE is a key tool for achieving this.

4. Immersive virtual environments provides a challenging and engaging means to explore space whether it be physical or digital.

Experimenting with different materials and expansion of coding in order to enhance the presence of people in architecture will be the next step forward with this design research. 


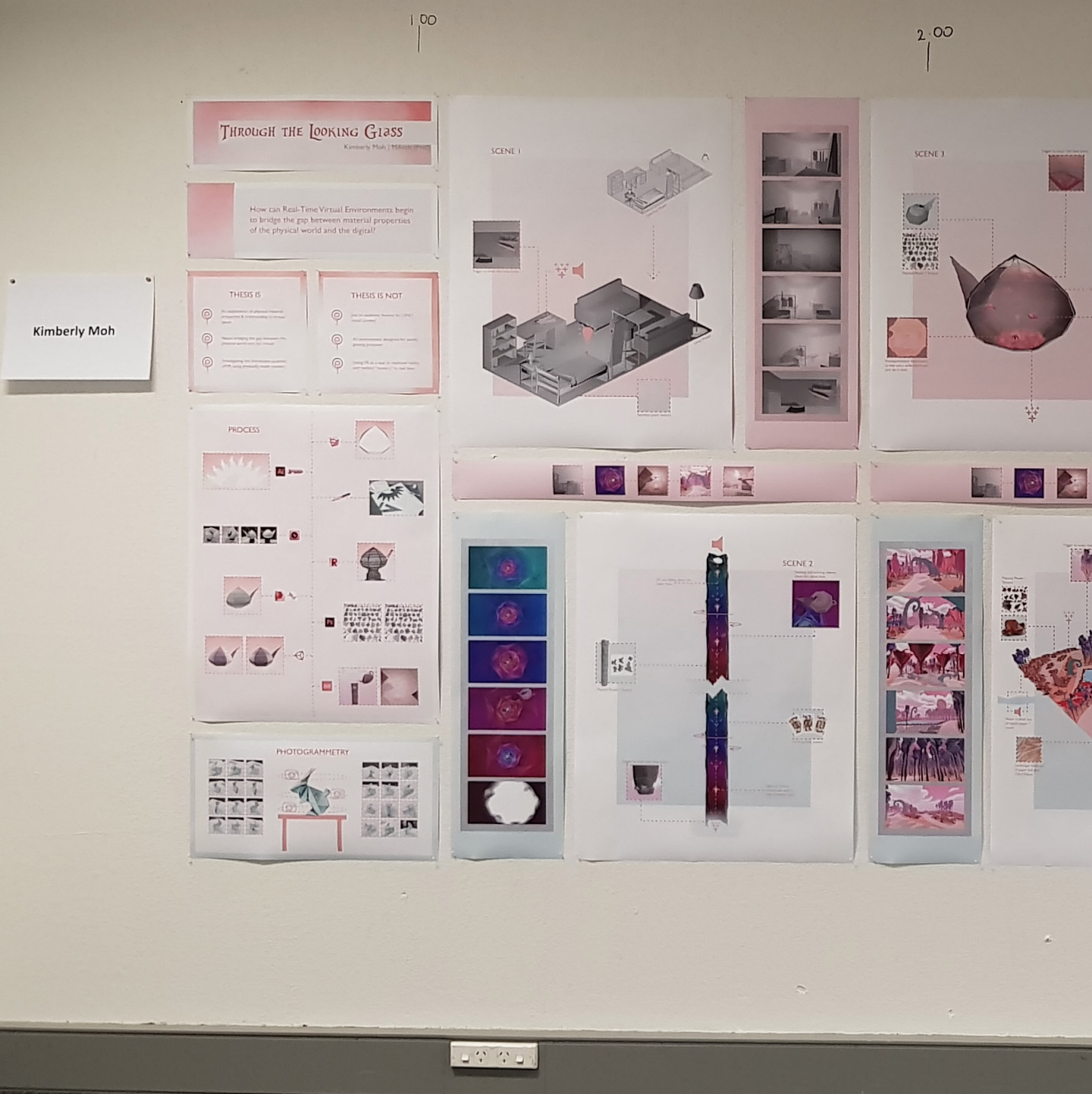

Fig.74. Image of the Final Review 


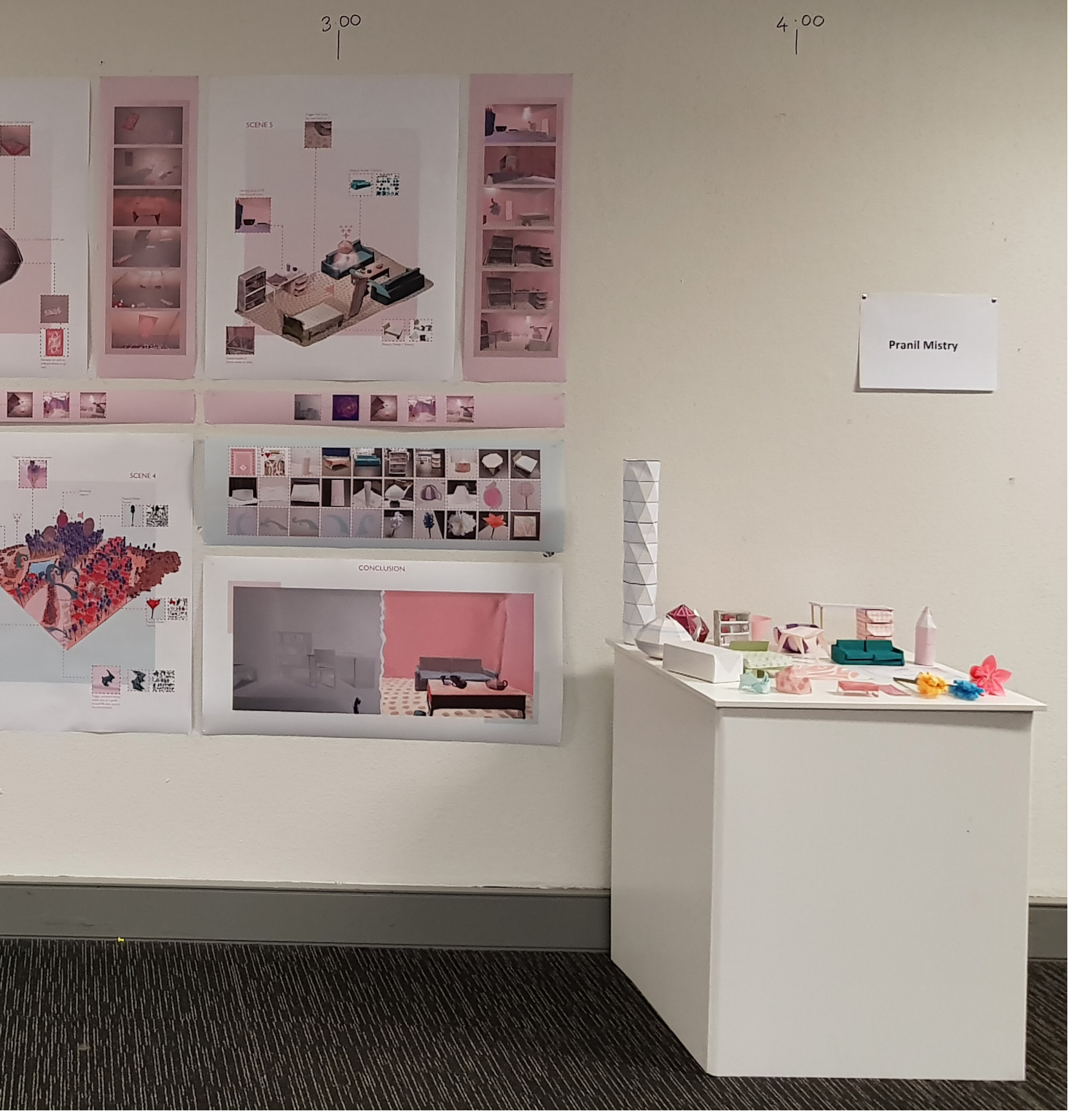


Allen, S. (2009). Practice: Architecture, Technique + Representation (2nd ed.).Abingdon, England: Routledge.

Cameron, P. (2015). Road to the IGF: State of Play's Lumino City: Gamasutra. Retrieved from https://www.gamasutra.com/view/news/237287/Road_to_ the_IGF_State_of_Plays_Lumino_City.php

Cheng, N. Y. W. (1995). Linking the Virtual to Reality: CAD \& Physical Modeling. In The Global Design Studio-Sixth International Conference on Computer Aided Architectural Design Futures (CAAD Futures), Centre for Advanced Studies in Architecture National University of Singapore, Singapore(pp. 303-311).

Fuente Suárez, L. A. D. L. (2016). Towards experiential representation in architecture. Journal of Architecture and Urbanism, 40(1), 47-58.

Hadjri, K. (2003). Bridging the gap between physical and digital models in architectural design studios. International Archives of the Photogrammetry, Remote Sensing and Spatial Information Sciences, 34(5), W10.

Holl, S., Pallasmaa, J., \& Perez-Gomez, A. (2006). Questions of Perception: Phenomenology of Architecture. San Francisco: William Stout Publishers.

Jakimowicz, A. (2002). The Physical and the Digital In Designing Architecture-Do They Really Meet?. In eCAADe(Vol. 20, pp. 17-21).

Knuuttila, T. (2005). Models, representation, and mediation. Philosophy of Science, 72(5), 1260-1271. doi: 10.1086/508124

Lessiter, J., Freeman, J., Keogh, E., \& Davidoff, J. (2001). A cross-media presence questionnaire: The ITC-Sense of Presence Inventory. Presence: Teleoperators and virtual environments, 10(3), 282-297.

Moleta, T. (2017). Digital Ephemera - Autonomous Real-Time Events in Virtual Environments. Protocols, Flows and Glitches, Proceedings of the 22nd International Conference of the Association for Computer-Aided Architectural Design Research in Aisa (CAARDIA) (pp. 13 -23). Retrieved from https://www.researchgate.net/ publication/316470721_Digital_Ephemera_-_Autonomous_Real-Time_Events_ in_Virtual_Environments 
Morris, M. (2006). Models:Architecture and the Miniature. Chichester, England: Wiley-Academy.

Openshaw, J. (2015). Postdigital Artisans:Craftsmanship with a New Aesthetic in Fashion, Art, Design and Architecture. Amsterdam, Netherlands: Frame Publishers.

Pallasmaa, J. (2009). The Thinking Hand: Existential and Embodied Wisdom in Architecture. Chichester, England: John Wiley \& Sons Ltd.

Pallasmaa, J. (2014). Empathic imagination: Formal and experiential projection. Architectural Design, 84(5), 80-85.

Photogrammetry. (n.d.) In Oxford Dictionaries. Retrieved from https:// en.oxforddictionaries.com/definition/photogrammetry

Schwarz, R. (2013). 10 Creepy examples of the uncanny valley: Stranger Dimensions. Retrieved from https://www.strangerdimensions.

com/2013/11/25/10-creepy-examples-uncanny-valley/

Smith, A. C. (2004). Architectural Model as Machine. Oxford, United Kingdom: Architectural Press.

Stavric, M., Sidanin, P., \& Tepavcevic, B. (2013). Architectural Scale Models in the Digital Age: design, representation and manufacturing. Austria: Springer Wien New York.

Sun, L., Fukuda, T., Tokuhara, T., \& Yabuki, N. (2014). Differences in spatial understanding between physical and virtual models. Frontiers of Architectural Research, 3(1), 28-35.

Webster, A. (2014). Lumino City- Paper Video Game: The Verge. Retrieved from https://www.theverge.com/2014/11/25/7275969/lumino-city-paper-videogame 
All figures not listed are the author's own

Fig.01 Schmidt Hammer Lassen Architects. (2017). CaoHeJing Technology HQ. Retrieved from: http://www.shl.dk/caohejing-guigu-creativeheadquarters/

Fig.02 Hufton and Crow. (2017). BIG - Via 57 West. Retrieved from: http://www.huftonandcrow.com/projects/gallery/via-57-west/

Fig.05 Sun, L., Fukuda, T., Tokuhara, T., \& Yabuki, N. (2014). Differences in spatial understanding between physical and virtual models. Frontiers of Architectural Research, 3(1), p.30

Fig.06 Murphy, Mekado. (2014). The Grand Budapest Hotel in Miniature. Retrieved from: https://www.nytimes.com/2014/03/02/movies/theminiature-model-behind-the-grand-budapest-hotel.html?_r=0\#

Fig.07 Webster, Andrew. (2014). Lumino City Screenshot. Retrieved from: https://www.theverge.com/2014/11/25/7275969/lumino-city-papervideo-game

Fig.08 Nyamyam. (2014). Tengami Screenshot. Retrieved from: https:// www.tengami.com/

Fig.09 Leonhardt, John /Laika Inc. (2014). Florian Perinelle works with the Lord Portley-Rind. Retrieved from: https://io9.gizmodo.com/how-boxtrollscreated-one-of-the-richest-worlds-of-any-1638716815 
Sciretta, Peter. (2012). Paranorman Set. Retrieved from: http:// www.slashfilm.com/laika-3d-color-printers-create-stopmotion-animatedmovie-paranorman-50-learned-set/

Fig.11 Robinson, Tasha. (2016). Kubo and the Two Strings set. Retrieved from: https://www.theverge.com/2016/8/18/12500814/laika-studiosbehind-the-scenes-kubo-and-the-two-strings-video

Fig.12 Theobald, Lewis. (2012). Matilda, Cambridge Theatre. Retrieved from: https://www.madebymagnitude.com/blog/a-chaotic-london-weekend/

Fig.13 Krulwich, Sara. (2013). The King and I production. Retrieved from: https://www.nytimes.com/2015/12/20/theater/the-stages-bestmoments-this-year.html

Fig.14 (2014). American Idiot Stage. Retrieved from: https://imgur. com/gallery/9MQPK

Fig.15 Leon, Linda. (2013). Martha. Retrieved from: http://www. lindaleonart.com/stage-design/

Fig.16 Great Lakes Scenic Studios. (2015). Chicago Shakespeare Theatre's Sense and Sensibility. Retrieved from: http://www.greatlakesscenic. com/Theatre/chicago-shakespeare-theatre-s-sense-and-sensibility.html 
Appendix 


\section{Load Level}

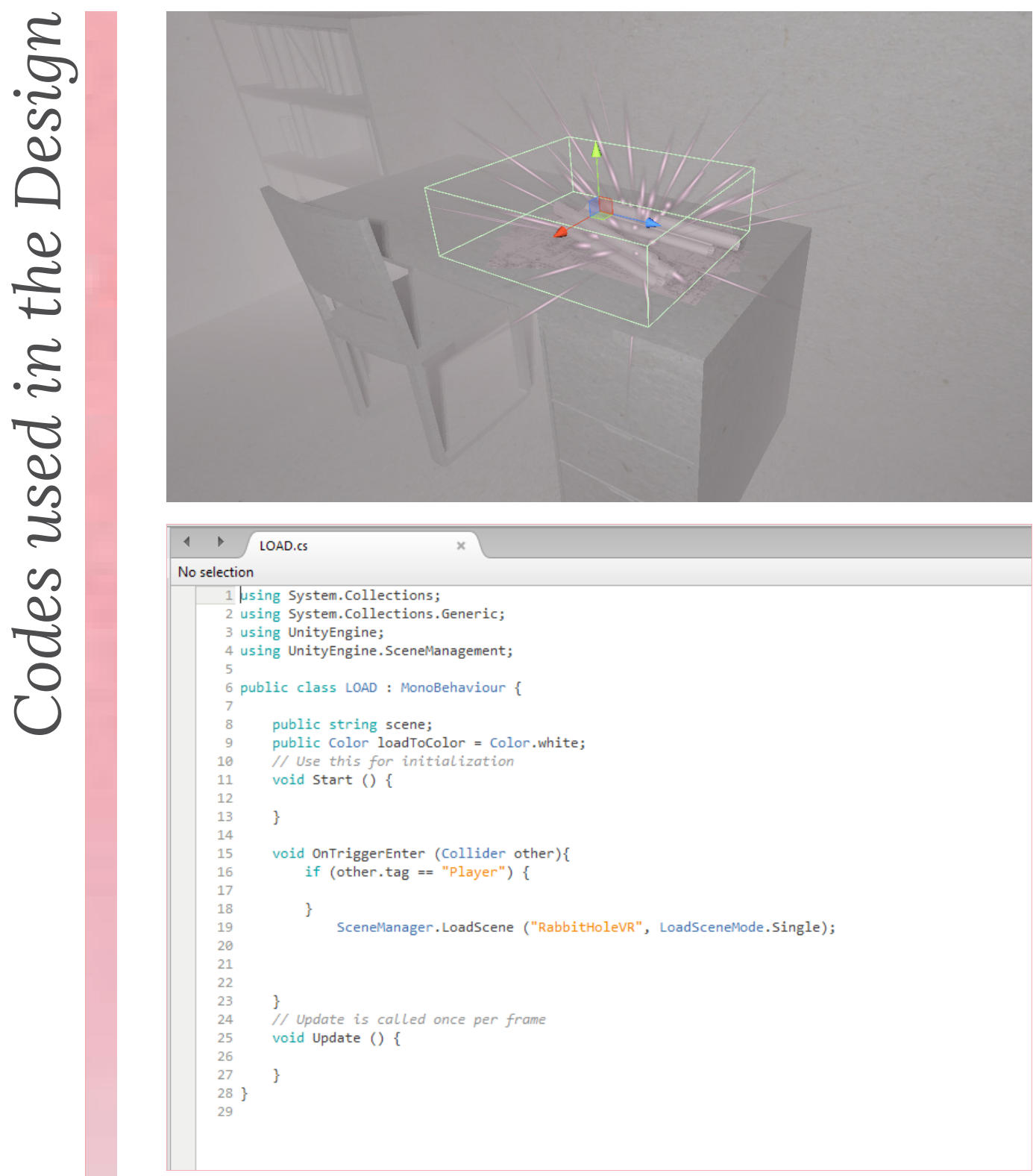

The Code to load to the next level. Replace the "RabbitHoleVR" to whatever name of the scene you want to load. Place this on the trigger box in Unity and once the 'Player' interacts with the box it will trigger the script prompting it to load the next scene. 


\section{Gravity Change}
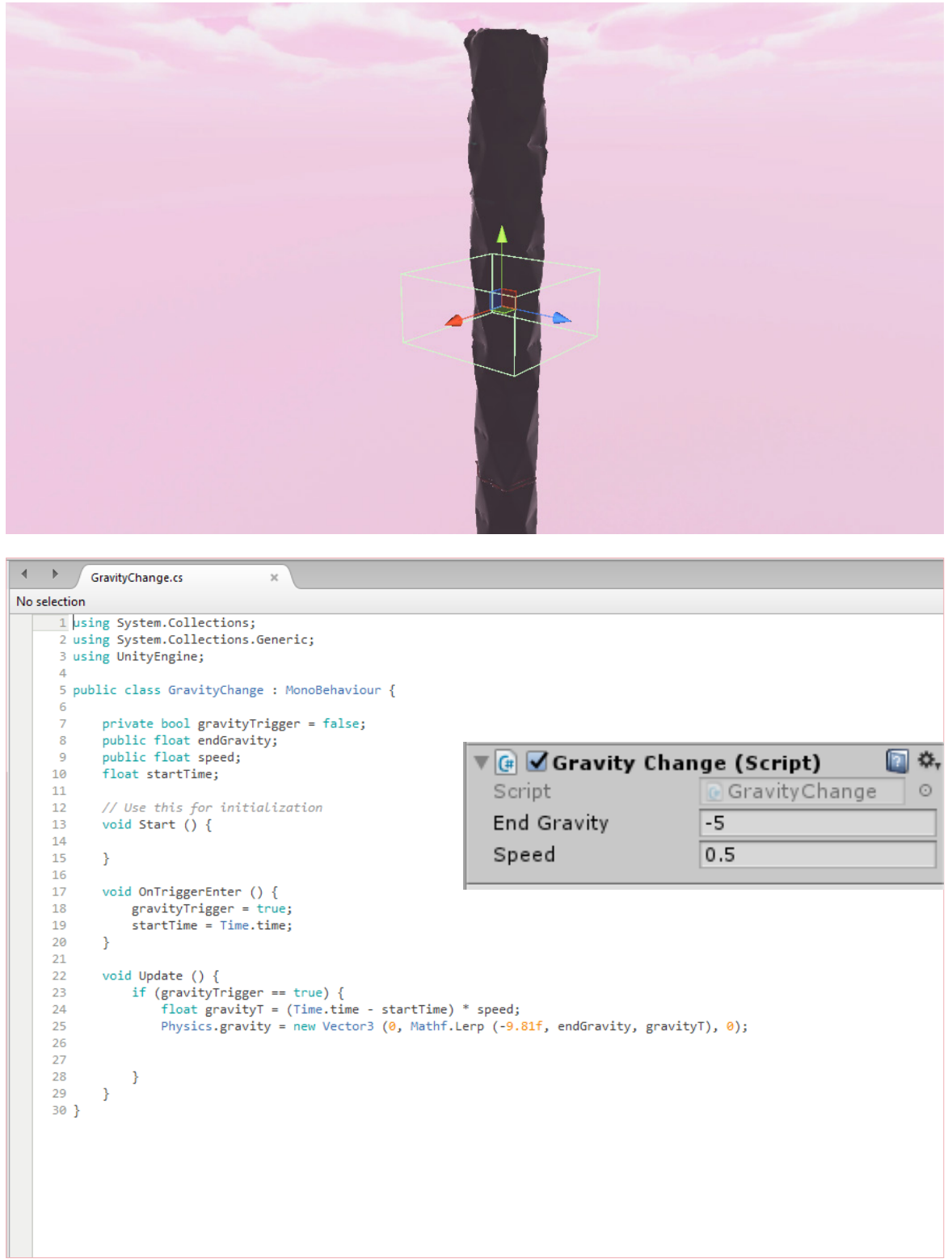

The Code to change the gravity when the 'player' falls through the trigger box. Depending on the number placed on the gravity and speed box it will either speed up or slow down the person when they are falling through the rabbit hole. 
Scale in VR

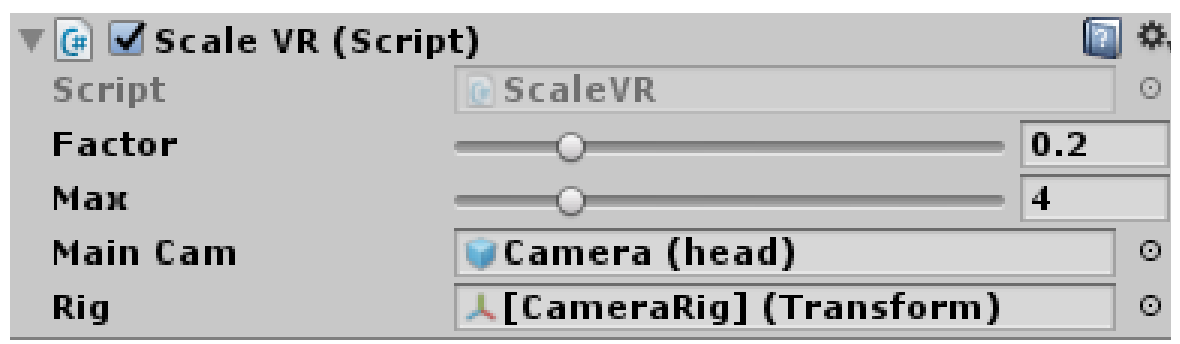

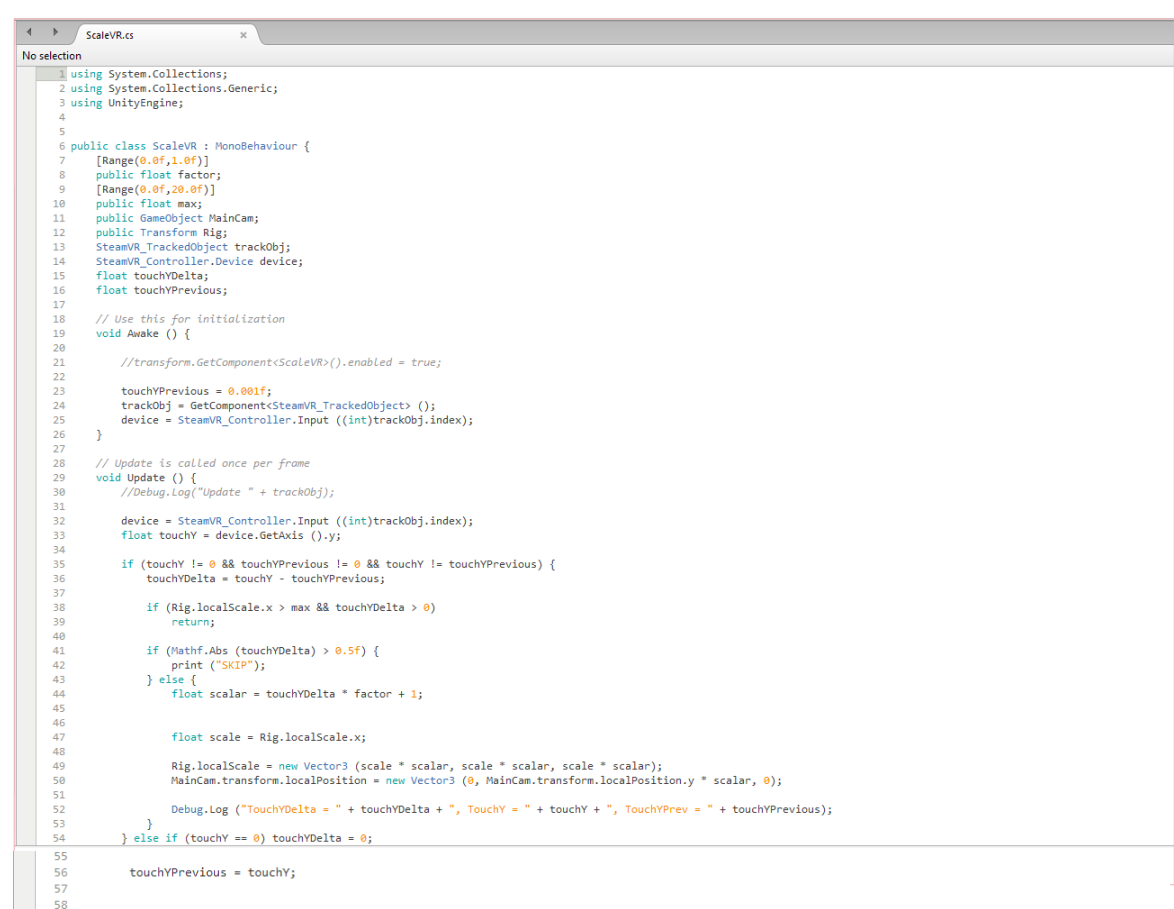

This code is place on the VR controller in Unity. It allows for the player to grow and shrink by swiping up or down on the track pad. 


\section{Bunny Movement}

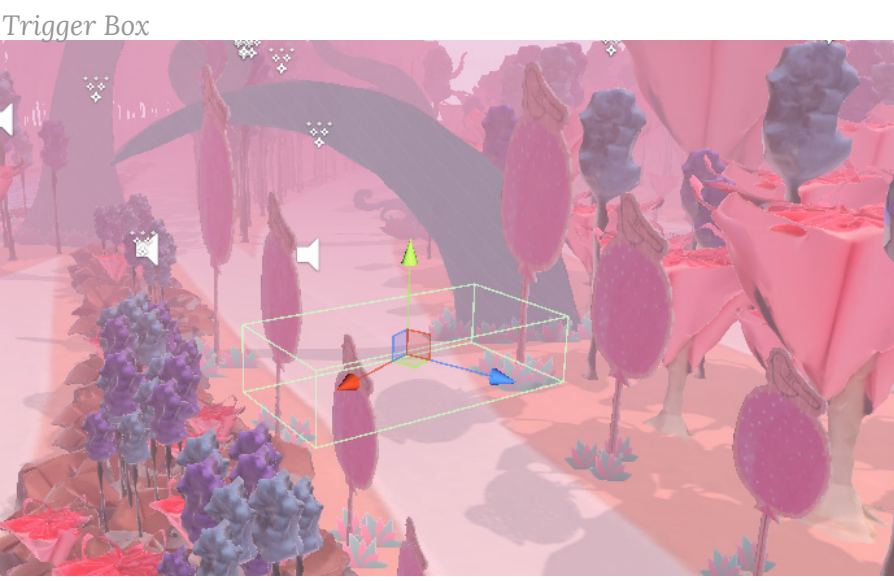

\begin{tabular}{|c|c|}
\hline $4>$ & TriggerMovement.cs \\
\hline \multicolumn{2}{|c|}{ No selection } \\
\hline \multicolumn{2}{|c|}{1 using System.Collections; } \\
\hline \multicolumn{2}{|r|}{2 using System.Collections.Generic; } \\
\hline \multicolumn{2}{|r|}{3 using UnityEngine; } \\
\hline \multicolumn{2}{|c|}{4 using UnityEngine.AI; } \\
\hline \multicolumn{2}{|c|}{5} \\
\hline \multirow{2}{*}{\multicolumn{2}{|c|}{$\begin{array}{l}6 \text { public class TriggerMovement : MonoBehaviour }\{ \\
7\end{array}$}} \\
\hline & \\
\hline 8 & // Use this for initialization \\
\hline 9 & public Gameobject Bunny; \\
\hline 10 & NavMeshAgent agent; \\
\hline 11 & public Transform target; \\
\hline 12 & MovementBunny script; \\
\hline \multicolumn{2}{|r|}{ ex } \\
\hline \multicolumn{2}{|l|}{14} \\
\hline 15 & void Start () \{ \\
\hline \multicolumn{2}{|r|}{ agent $=$ Bunny. GetComponent $\langle$ NavMeshAgent $\rangle()$; } \\
\hline \multirow{2}{*}{\multicolumn{2}{|c|}{$\begin{array}{l}17 \\
18\end{array}$}} \\
\hline & \\
\hline \multirow{2}{*}{\multicolumn{2}{|c|}{$\begin{array}{l}19 \\
20\end{array}$}} \\
\hline & \\
\hline 21 & void OnTriggerEnter (Collider other) \{ \\
\hline 22 & if (other.tag $==$ "Player") \{ \\
\hline 23 & \\
\hline 24 & agent.SetDestination (target.position); \\
\hline $\begin{array}{l}25 \\
26\end{array}$ & \} \\
\hline
\end{tabular}

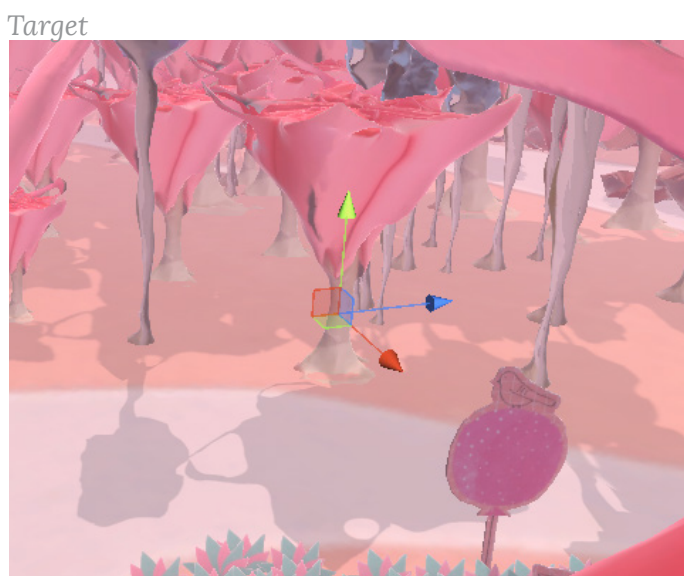

\begin{tabular}{|c|c|c|}
\hline \multicolumn{2}{|c|}{$\nabla$ ఏ $\checkmark$ Trigger Movement (Script) } & [0] \\
\hline Script & Q TriggerMovement & $\odot$ \\
\hline Bunny & BunnyNAV & $\odot$ \\
\hline Target & Ltarget (Transform) & $\odot$ \\
\hline
\end{tabular}

This is the code that allows the bunny to move ahead of the player as a guide around the forest scene (Scene $4)$. Once the player enters the trigger box the bunny will start to move to the designated target location. This can be done multiple times with different trigger boxes. 


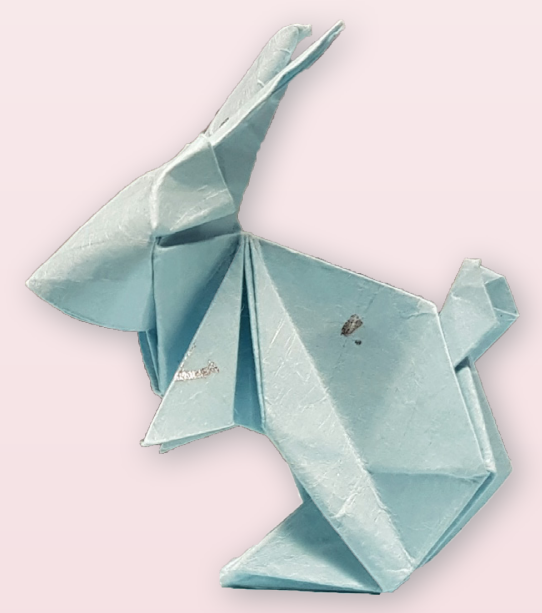

